\title{
Search for massive resonances decaying into pairs of boosted bosons in semi-leptonic final states at $\sqrt{s}=8 \mathrm{TeV}$
}

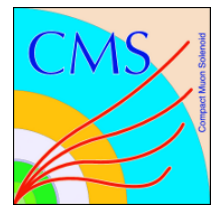

\section{The CMS collaboration}

E-mail: cms-publication-committee-chair@cern.ch

ABSTRACT: A search for new resonances decaying to WW, ZZ, or WZ is presented. Final states are considered in which one of the vector bosons decays leptonically and the other hadronically. Results are based on data corresponding to an integrated luminosity of $19.7 \mathrm{fb}^{-1}$ recorded in proton-proton collisions at $\sqrt{s}=8 \mathrm{TeV}$ with the CMS detector at the CERN LHC. Techniques aiming at identifying jet substructures are used to analyze signal events in which the hadronization products from the decay of highly boosted $\mathrm{W}$ or $\mathrm{Z}$ bosons are contained within a single reconstructed jet. Upper limits on the production of generic WW, ZZ, or WZ resonances are set as a function of the resonance mass and width. We increase the sensitivity of the analysis by statistically combining the results of this search with a complementary study of the all-hadronic final state. Upper limits at $95 \%$ confidence level are set on the bulk graviton production cross section in the range from 700 to $10 \mathrm{fb}$ for resonance masses between 600 and $2500 \mathrm{GeV}$, respectively. These limits on the bulk graviton model are the most stringent to date in the diboson final state.

KEYwords: Hadron-Hadron Scattering, Particle and resonance production, Jet substructure

ARXIV EPRINT: 1405.3447 


\section{Contents}

1 Introduction $\quad 2$

2 The CMS detector 4

3 Simulated samples $\quad 4$

4 Reconstruction and selection of events 5

4.1 Trigger and basic offline selection 5

4.2 Muon reconstruction and selection 6

$\begin{array}{lll}4.3 & \text { Electron reconstruction and selection } & 6\end{array}$

$\begin{array}{lll}4.4 & \text { Jets and missing transverse energy reconstruction } & 7\end{array}$

4.5 $\mathrm{W} \rightarrow \ell \nu$ and $\mathrm{Z} \rightarrow \ell \ell$ reconstruction and identification 8

4.6 $\mathrm{W} \rightarrow \mathrm{q} \overline{\mathrm{q}}^{\prime}$ and $\mathrm{Z} \rightarrow \mathrm{q} \overline{\mathrm{q}}$ identification using jet substructure 8

4.7 Final event selection and categorization 9

$\begin{array}{lll}5 & \text { W tagging in a top-quark enriched control sample } & 10\end{array}$

6 Modeling of background and signal $\quad 12$

$\begin{array}{ll}6.1 \text { Background estimation } & 12\end{array}$

$\begin{array}{ll}\text { 6.2 Modeling of the signal mass distribution } & 17\end{array}$

7 Systematic uncertainties $\quad 18$

$\begin{array}{lll}7.1 & \text { Systematic uncertainties in the background estimation } & 18\end{array}$

$\begin{array}{lll}7.2 & \text { Systematic uncertainties in the signal prediction } & 18\end{array}$

8 Statistical interpretation $\quad 19$

8.1 Limits on a narrow-width bulk graviton model 20

$\begin{array}{ll}8.2 \text { Model-independent limits } & 21\end{array}$

9 Summary 26

A Detailed instructions and additional material for generic interpretation $\begin{array}{ll}\text { of the results } & \mathbf{2 8}\end{array}$

The CMS collaboration $\quad 41$ 


\section{Introduction}

The standard model (SM) of particle physics has been very successful in describing the high-energy physics phenomena investigated so far. One of the predictions of the SM is the existence of a scalar particle, known as the Higgs boson, associated with the spontaneous breaking of the electroweak (EW) symmetry and responsible for the masses of the SM particles [1-6]. The recent discovery by the ATLAS and CMS Collaborations of a particle compatible with the SM predictions for the Higgs boson provides further verification of the SM [7-9]. In view of large loop corrections to the Higgs boson mass, the question arises whether the measured Higgs boson mass is the result of fine-tuned constants of nature within the SM or whether new physics at the TeV scale stabilizes the Higgs field vacuum. This question can be reformulated in terms of the large difference between the mass of the Higgs boson and the Planck scale $M_{\mathrm{Pl}}$, where the gravitational force is expected to have the same strength as the other fundamental forces $\left(M_{\mathrm{Pl}} \sim 10^{16} \mathrm{TeV}\right)$.

In many theoretical extensions of the SM, the spontaneous breaking of the EW symmetry is associated with new strong dynamics appearing at the $\mathrm{TeV}$ scale. For instance, the origin of the new dynamics may be due to new interactions [10-12] or a composite Higgs boson [13-15]. These extensions of the SM predict the existence of new resonances coupling to pairs of massive vector bosons ( $\mathrm{VV}$, where $\mathrm{V}=\mathrm{W}$ or $\mathrm{Z}$ ). Results from previous direct searches at CMS [16-19] and ATLAS [20-23], and from indirect bounds from the EW sector and from flavor physics $[24,25]$ generally place lower limits on the masses of these $\mathrm{VV}$ resonances above the $\mathrm{TeV}$ scale.

Models extending the number of spatial dimensions are of particular interest in the attempt to explain the apparently large difference between the EW and the gravitational scale. Some of these models predict the existence of a so-called tower of Kaluza-Klein (KK) excitations of a spin- 2 boson, the KK graviton. The WW and ZZ channels are some of the possible decay modes of the Randall-Sundrum (RS) graviton [26] in warped extra dimension models. The original RS model (here denoted as RS1) can be extended to the bulk graviton $\left(G_{\text {bulk }}\right)$ model, which addresses the flavor structure of the SM through localization of fermions in the warped extra dimension [27-29]. In this scenario, coupling of the graviton to light fermions is highly suppressed and the decays into photons are negligible. On the other hand, the production of gravitons from gluon fusion and their decays into a pair of massive gauge bosons can be sizable at hadron colliders. The model has two free parameters: the mass of the first mode of the KK bulk graviton, $\mathrm{M}_{\mathrm{G}}$, and the ratio $k / \bar{M}_{\mathrm{Pl}}$, where $k$ is the unknown curvature scale of the extra dimension, and $\bar{M}_{\mathrm{Pl}} \equiv M_{\mathrm{Pl}} / \sqrt{8 \pi}$ is the reduced Planck mass. Previous direct searches set limits on the cross section times branching fraction for the production of $\mathrm{G}_{\mathrm{bulk}}$ as a function of $\mathrm{M}_{\mathrm{G}}[17,22]$. It should be noted that a revised version of the theoretical calculations has been recently released, superseding the previous one $[27,30]$. With the new calculation, which predicts production cross sections four times smaller, previous limits may have to be revised. For example, the lower limit on the graviton mass quoted in ref. [17] is affected, though the experimental bounds on the graviton production cross section times branching fraction as a function of mass remain valid. This paper supersedes results from ref. [17] for graviton masses 

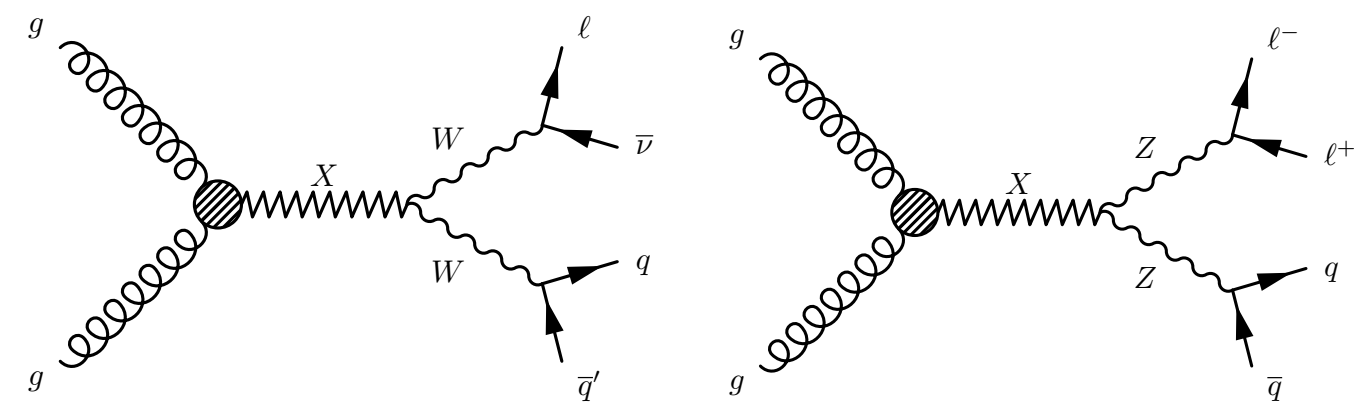

Figure 1. Two Feynman diagrams for the production of a generic resonance $\mathrm{X}$ decaying to some of the final states considered in this study.

above $600 \mathrm{GeV}$, while the limit on the production cross section for graviton masses below $600 \mathrm{GeV}$ from ref. [17] remains the most stringent CMS result for the final state considered in the reference.

We present a search for new resonances decaying to WW, ZZ, or WZ in which one of the bosons decays leptonically and the other hadronically. The analysis is based on the proton-proton collision data at $\sqrt{s}=8 \mathrm{TeV}$ collected by the CMS experiment at the CERN Large Hadron Collider (LHC) during 2012 and corresponding to an integrated luminosity of $19.7 \mathrm{fb}^{-1}$. The final states considered are either $\ell \nu \mathrm{q} \overline{\mathrm{q}}^{(\prime)}$ or $\ell \ell \mathrm{q} \overline{\mathrm{q}}^{(\prime)}$, resulting in events with a charged lepton, a neutrino and a single reconstructed-jet $(\ell \nu+\mathrm{V}$-jet channel) or two charged leptons and a single reconstructed-jet ( $\ell \ell+\mathrm{V}$-jet channel). Figure 1 shows two Feynman diagrams relevant to the production and decays of a generic resonance $\mathrm{X}$. The search is limited to final states where $\ell=\mu$ or e; however the results include the case in which $\mathrm{W} \rightarrow \tau \nu$ or $\mathrm{Z} \rightarrow \tau \tau$ where the tau decay is $\tau \rightarrow \ell \nu \nu$. The gain in sensitivity from the decay channels including $\tau$ leptons is limited, because of the small branching ratios involved.

For large values of the resonance mass, the two quarks originating from the hadronically decaying $\mathrm{W}$ or $\mathrm{Z}$ bosons are highly collimated and are typically reconstructed as a single massive jet ("V jet"). Final states where two jets from a V decay are well resolved in the detector give a negligible contribution to the sensitivity for the resonance masses considered in this search. This analysis uses the additional information from jet substructure to perform jet "V tagging" and to further suppress the SM background, which mainly originates from the SM production of $\mathrm{V}+$ jets and non-resonant $\mathrm{VV}$ events [31]. In the $\ell \nu+\mathrm{V}$-jet channel t $\overline{\mathrm{t}}$ events also contribute to the background. The signal is characterized as a local enhancement in the WW, ZZ, or WZ invariant mass distribution $\left(m_{\mathrm{VV}}\right)$. The invariant mass of the WW system is determined by estimating the neutrino transverse momentum with the measured missing transverse energy $\left(E_{\mathrm{T}}^{\mathrm{miss}}\right)$ in the event, while an estimate of the neutrino longitudinal momentum is derived by imposing the constraint of the $\mathrm{W}$ mass on the invariant mass of the $\ell \nu$ system. The mass distributions for the dominant $\mathrm{W}+$ jets and $\mathrm{Z}+$ jets backgrounds are determined from events with a reconstructed jet mass not compatible with the $\mathrm{W}$ or $\mathrm{Z}$ hypothesis. This analysis is optimized for $\mathrm{WW}$ and $\mathrm{ZZ}$ resonances, but because of the loose requirement on the $\mathrm{V}$-jet mass it is also sensitive to charged resonances decaying to WZ. 
The results of this analysis are combined with limits derived in a companion CMS search for resonances decaying to VV final states in the all-hadronic decay channel [32]. The all-hadronic analysis uses the same $\mathrm{V}$-tagging techniques as presented here to separate the signal from the large multijet background.

In this paper, section 2 briefly describes the CMS detector; section 3 gives an overview of the simulations used in this analysis. Section 4 provides a detailed description of the reconstruction and event selection. In section 5 we demonstrate the performance of the $\mathrm{V}$ tagging by studying a sample of events enriched in top quarks. Section 6 describes the background estimation and the signal modeling. Systematic uncertainties are discussed in section 7. The results of the search for a bulk graviton and for generic resonances are presented in section 8. Appendix A contains detailed instructions for applying the results presented here to new models with diboson resonances.

\section{The CMS detector}

The central feature of the CMS detector is a $3.8 \mathrm{~T}$ superconducting solenoid with a $6 \mathrm{~m}$ internal diameter. Within the field volume are the silicon tracker, the crystal electromagnetic calorimeter (ECAL), and the brass and scintillator hadron calorimeter (HCAL). The calorimeters are supplemented by a steel/quartz-fiber Cherenkov detector (HF) to extend the calorimetric coverage in the forward direction. The muon system is installed outside the solenoid and embedded in the steel flux-return yoke. The CMS experiment uses a right-handed coordinate system, with the origin at the nominal collision point, the $x$-axis pointing to the center of the LHC ring, the $y$-axis pointing up (perpendicular to the LHC plane), and the $z$-axis along the anticlockwise beam direction. The polar angle $(\theta)$ is measured from the positive $z$-axis and the azimuthal angle $(\phi)$ is measured from the positive $x$-axis in the $x-y$ plane. The radius $(r)$ denotes the distance from the $z$-axis and the pseudorapidity $(\eta)$ is defined as $\eta=-\ln [\tan (\theta / 2)]$. The CMS tracker consists of 1440 silicon pixel and 15148 silicon strip detector modules. The ECAL consists of nearly 76000 lead tungstate crystals, which provide coverage in pseudorapidity $|\eta|<1.479$ in the central barrel region and $1.479<|\eta|<3.0$ in the two forward endcap regions. The HCAL consists of a sampling calorimeter which utilizes alternating layers of brass as an absorber and plastic scintillator as an active material, covering the pseudorapidity range $|\eta|<3$, which is extended to $|\eta|<5$ in combination with the HF. Muons are measured in the pseudorapidity range $|\eta|<2.4$, with detection planes which employ three technologies: drift tubes, cathode strip chambers, and resistive plate chambers. A detailed description of the CMS detector can be found in ref. [33].

\section{Simulated samples}

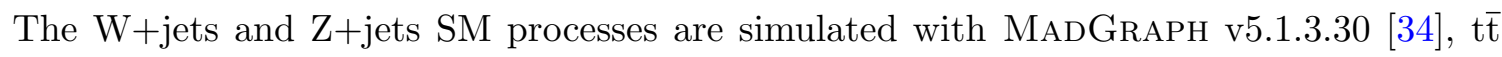
and single top quark events are generated with POWHEG 1.0 r1380 [35-40], while diboson (WW, WZ, and ZZ) processes are produced with PYTHIA v6.424 [41]. The parton showering and hadronization are performed with PYTHIA using the Z2* tune [42]. The CTEQ6L [43] 
parton distribution functions (PDF) are used in all generated samples, except for the POWHEG t $\overline{\mathrm{t}}$ sample, where the CT10 PDF set [44] is used. All generated samples are processed through a GEANT4-based [45] simulation of the CMS detector. The simulated background samples are normalized using inclusive cross sections calculated at next-toleading order (NLO), or next-to-next-to-leading order (NNLO) where available, calculated with MCFM v6.6 [46-49] and FEWZ v3.1 [50].

The bulk graviton model is used as a benchmark signal process, with the graviton forced to decay to the $\mathrm{WW}$ and $\mathrm{ZZ}$ final states. In this specific model, the vector gauge bosons are produced with a longitudinal polarization $\left(\mathrm{V}_{\mathrm{L}}\right)$ in more than $99 \%$ of the cases. The graviton masses considered lie in the range 600 to $2500 \mathrm{GeV}$. The events are generated with JHUGEN v3.1.8 [51], which properly treats the spin correlations in the final state, while the values for the bulk graviton cross sections and decay rates are calculated at leading order with CALCHEP v3.4.1 [52]. The total cross section of the process pp $\rightarrow \mathrm{G}_{\text {bulk }}$ at $\sqrt{s}=8 \mathrm{TeV}$ is $15.1 \mathrm{fb}$, for a graviton mass of $1 \mathrm{TeV}$ and $k / \bar{M}_{\mathrm{Pl}}=0.5$. At the same resonance mass, the branching fraction of $\mathrm{G}_{\text {bulk }} \rightarrow \mathrm{WW}\left(\mathrm{G}_{\mathrm{bulk}} \rightarrow \mathrm{ZZ}\right)$ is $18.7 \%(9.5 \%)$.

Supplementary minimum bias interactions are added to the generated events in order to match the additional particle production observed in data from the large number of protonproton interactions occurring per LHC bunch crossing (pileup). The simulated samples are corrected for observed differences between data and simulation in the efficiencies of lepton trigger, lepton identification/isolation, and selection criteria identifying jets originating from hadronization of $b$ quarks ( $b$ jets).

\section{Reconstruction and selection of events}

\subsection{Trigger and basic offline selection}

In the $\ell \nu+\mathrm{V}$-jet channel, candidate signal events are selected online with a trigger requiring either one muon or one electron, without isolation requirements and with loose identification criteria. The transverse momentum $\left(p_{\mathrm{T}}\right)$ measured online must be higher than $40 \mathrm{GeV}$ for the muons while the minimum transverse energy threshold is $80 \mathrm{GeV}$ for the electrons. The trigger efficiencies for the single-muon trigger vary between $82 \%$ and $94 \%$ depending on the value of the $\eta$ of the muon. The efficiency is above $98 \%$ for the single-electron trigger.

In the $\ell \ell+\mathrm{V}$-jet channel, events are selected online with a trigger requiring either two muons or two electromagnetic energy deposits, with loose identification criteria. The trigger used for the electron channel rejects candidates if there is significant energy in the HCAL associated with the ECAL cluster. The clusters are required to be loosely matched to the trajectories of tracks with associated hits in the pixel detector. No lepton isolation requirements are applied at the trigger level. The $p_{\mathrm{T}}$ thresholds applied in the online selection of the muons are $22 \mathrm{GeV}$ for the highest- $p_{\mathrm{T}}$ muon and $8 \mathrm{GeV}$ for the second highest- $p_{\mathrm{T}}$ muon. The transverse energy threshold for the ECAL clusters is set at $33 \mathrm{GeV}$. The trigger efficiency of the double-muon trigger varies between $80 \%$ and $98 \%$ depending on the value of $\eta$ of the leptons. The efficiency of the double-electron trigger is above $99 \%$.

Offline, all events are required to have at least one primary vertex reconstructed within a $24 \mathrm{~cm}$ window along the beam axis, with a transverse distance from the nominal pp 
interaction region of less than $2 \mathrm{~cm}$ [53]. In the presence of more than one vertex passing these requirements, the primary-event vertex is chosen to be the one with the highest total $p_{\mathrm{T}}^{2}$, summed over all the associated tracks.

\subsection{Muon reconstruction and selection}

Tracker muons are reconstructed using the inner tracker with an additional requirement of a matching hit in the muon system [54]. Tracker muons must satisfy requirements on the impact parameter of the track and on the number of hits in the silicon tracker. Muons reconstructed with a fit using both the inner tracking system and the muon spectrometer are defined as global muons [54]. Compared to tracker muons, global muons must pass additional requirements on the number of hits in the muon detectors. These quality selections ensure a precise measurement of the four-momentum and reject misreconstructed muons. A large fraction of isolated high- $p_{\mathrm{T}}$ muons is usually identified as both tracker and global muons. For large values of the mass of a ZZ resonance, the two charged leptons originating from the high- $p_{\mathrm{T}} \mathrm{Z}$ boson are highly collimated because of the large Lorentz boost and are characterized by small values of their angular separation, $\Delta R=\sqrt{(\Delta \eta)^{2}+(\Delta \phi)^{2}}$. While the global muon reconstruction and identification are optimized for the case of wellseparated muons, inefficiencies in the global-muon reconstruction are observed when two muons from a boosted $\mathrm{Z}$ are very close, typically causing the loss of one of them. In order to recover the inefficiency in the muon identification, the $\ell \ell+V$-jet selection requires two tracker muons of which at least one should be reconstructed and identified as a global muon. Wherever possible, the kinematic quantities are calculated with the global fit.

An isolation requirement is applied in order to suppress the background from multijet events where jet constituents are identified as muons. A cone of radius $\Delta R=0.3$ is constructed around the muon direction. The isolation parameter is defined as the scalar sum of the transverse momenta of all the additional reconstructed tracks within the cone, divided by the muon $p_{\mathrm{T}}$. The contribution from any other muon candidate in the cone is excluded from the computation in order to retain high signal efficiency when the two muons originate from a boosted $\mathrm{Z}$ and are collimated to the point of entering in each other's isolation cone. Muon candidates with an isolation parameter smaller than 0.1 are considered isolated and used in the rest of the analysis. The efficiency of this muon selection has been measured with a tag-and-probe method using $\mathrm{Z}$ bosons [55], and it has a negligible dependence on the number of reconstructed primary vertices in the event. In the $\ell \ell+V$-jet channel, events must have at least two muons with $|\eta|<2.4$ of which one should have $p_{\mathrm{T}}>40 \mathrm{GeV}$ and the other $p_{\mathrm{T}}>20 \mathrm{GeV}$. In the $\ell \nu+\mathrm{V}$-jet channel, we require exactly one global muon with $p_{\mathrm{T}}>50 \mathrm{GeV}$ and $|\eta|<2.1$.

\subsection{Electron reconstruction and selection}

Electron candidates are reconstructed by matching energy deposits in the ECAL with reconstructed tracks [56]. In order to suppress multijet background, electron candidates must pass stringent quality criteria tuned for high- $p_{\mathrm{T}}$ objects and an isolation selection [57]. The total scalar sum of the $p_{\mathrm{T}}$ of all the tracks in a cone of radius $\Delta R=0.3$ around the electron direction, excluding tracks within an inner cone of $\Delta R=0.04$ to remove the 
contribution from the electron itself, must be less than $5 \mathrm{GeV}$. A calorimetric isolation parameter is calculated by summing the energies of reconstructed deposits in both ECAL and HCAL, not associated with the electron itself, within a cone of radius $\Delta R=0.3$ around the electron. The upper threshold for this isolation parameter depends on the electron kinematic quantities and the average amount of additional energy coming from pileup interactions. When evaluating the isolation parameter in the $\ell \ell+\mathrm{V}$-jet channel, the contribution from any nearby electron candidate is excluded from the calculation. This is done in order to retain high signal efficiency when the two leptons from a $\mathrm{Z}$ decay are highly boosted and one of them enters into the isolation cone of the other. In the $\ell \ell+\mathrm{V}$-jet channel, we require at least two electrons with $p_{\mathrm{T}}>40 \mathrm{GeV}$ and $|\eta|<2.5$. In the $\ell \nu+\mathrm{V}$-jet channel, we require exactly one electron with $p_{\mathrm{T}}>90 \mathrm{GeV}$ and $|\eta|<2.5$. In both channels, the electrons must fall outside the overlap region between the ECAL barrel and endcaps $(1.44<|\eta|<1.56)$.

\subsection{Jets and missing transverse energy reconstruction}

Hadronic jets are clustered from the four-momenta of the particles reconstructed by the CMS particle-flow (PF) algorithm [58, 59], using the FASTJET software package [60]. The $\mathrm{PF}$ algorithm reconstructs individual particles by combining information from all subdetector systems. The reconstructed PF constituents are assigned to one of the five candidate categories (electrons, muons, photons, charged hadrons, and neutral hadrons). In the jet clustering procedure charged PF particles not associated with the primary-event vertex are excluded. Jets used for identifying the hadronically decaying $\mathrm{W}$ and $\mathrm{Z}$ bosons are clustered using the Cambridge-Aachen algorithm [61] with a distance parameter $R=0.8$ ("CA8 jets"). In order to identify b jets, the anti- $k_{\mathrm{T}}$ jet clustering algorithm is used [62] with a distance parameter $R=0.5$ ("AK5 jets") and the combined secondary vertex b-tagging algorithm [63] is applied to the reconstructed AK5 jets. The ratio of the b-tagging efficiency between data and simulation is used as a scale factor to correct the simulated events. A correction based on the projected area of the jet on the front face of the calorimeter is used to take into account the extra energy clustered in jets due to neutral particles coming from pileup. Jet energy corrections are derived from simulation and from dijet and photon + jet events in data [64]. Additional quality criteria are applied to the jets in order to remove spurious jet-like features originating from isolated noise patterns in the calorimeters or the tracker. The efficiency of these jet quality requirements for signal events is above $99 \%$. The CA8 (AK5) jets are required to be separated from any well-identified electron or muon by $\Delta R>0.8(0.3)$. All jets must have $p_{\mathrm{T}}>30 \mathrm{GeV}$ and $|\eta|<2.4$ in order to be considered in the subsequent steps of the analysis.

The missing transverse energy $E_{\mathrm{T}}^{\text {miss }}$ is defined as the magnitude of the vector sum of the transverse momenta of the reconstructed $\mathrm{PF}$ objects. The raw $E_{\mathrm{T}}^{\text {miss }}$ value is modified to account for corrections to the energy scale of all the reconstructed AK5 jets in the event. More details on the $E_{\mathrm{T}}^{\text {miss }}$ performance in CMS can be found in refs. [65, 66]. The requirement $E_{\mathrm{T}}^{\text {miss }}>40(80) \mathrm{GeV}$ is applied only for the muon (electron) channel in the $\ell \nu+\mathrm{V}$-jet analysis. The threshold is higher in the electron channel to further suppress the larger background from multijet processes. 


\section{5 $\mathrm{W} \rightarrow \ell \nu$ and $\mathrm{Z} \rightarrow \ell \ell$ reconstruction and identification}

In the $\ell \nu+\mathrm{V}$-jet channel, identified electrons or muons are associated with the $\mathrm{W} \rightarrow \ell \nu$ candidate. The transverse momentum of the undetected neutrino is assumed to be equal to the $E_{\mathrm{T}}^{\text {miss }}$. The longitudinal momentum of the neutrino is obtained by solving a secondorder equation that sets the $\ell \nu$ invariant mass to be equal to the known W-boson mass [67]. In the case of two real solutions, the smaller one is chosen; in the case of two complex solutions, their real part is used. The four-momentum of the neutrino is used to build the four-momentum of the $\mathrm{W} \rightarrow \ell \nu$ candidate. The same procedure is applied for $\mathrm{W} \rightarrow \tau \nu$ candidates, where the $\tau$ decays to one electron or muon and two neutrinos. In this case, the $E_{\mathrm{T}}^{\mathrm{miss}}$ represents the transverse momentum of the three-neutrino system.

In the $\ell \ell+\mathrm{V}$-jet channel, the leptonic Z-boson candidate is reconstructed by combining two oppositely charged lepton candidates of the same flavor. The invariant mass of the dilepton system is required to be between 70 and $110 \mathrm{GeV}$, consistent with the Zboson mass. This requirement is introduced to reduce significantly the Drell-Yan and top-quark backgrounds, at the cost of a suppression of the small $\mathrm{Z} \rightarrow \tau \tau \rightarrow(\ell \nu \nu)(\ell \nu \nu)$ signal contribution.

\section{6 $\mathrm{W} \rightarrow \mathrm{q} \overline{\mathrm{q}}^{\prime}$ and $\mathrm{Z} \rightarrow \mathrm{q} \overline{\mathrm{q}}$ identification using jet substructure}

CA8 jets are used to reconstruct the $\mathrm{W}$-jet and Z-jet candidates from hadronic decays of boosted $\mathrm{W}$ and $\mathrm{Z}$ bosons, respectively. In order to discriminate against multijet backgrounds we exploit both the reconstructed jet mass, which is required to be close to the $\mathrm{W}$ - or Z-boson mass, and the two-prong jet substructure produced by the particle cascades of two high- $p_{\mathrm{T}}$ quarks merging into one jet.

As the first step in exploring potential substructure, the jet constituents are subjected to a jet grooming algorithm, that improves the resolution on the jet mass and reduces the effect of pileup [68]. The goal of jet grooming is to re-cluster the jet constituents while applying additional requirements that eliminate soft, large-angle quantum chromodynamic (QCD) radiation coming from sources other than the hard interaction responsible for the $\mathrm{V}$ boson. Different jet grooming algorithms have been explored at CMS and their performance on jets in multijet processes has been studied in detail [68]. In this analysis, we use the jet pruning algorithm [69, 70]. Jet pruning reclusters each jet starting from all its original constituents using the CA algorithm, discarding "soft" recombinations in each step of the iterative CA procedure. The combination of two input four-vectors $i$ and $j$ is considered soft if either (i) $p_{\mathrm{T}}^{i}$ or $p_{\mathrm{T}}^{j}$ is small compared to the $p_{\mathrm{T}}$ of their combination, or (ii) the separation angle between $i$ and $j$ is large. With $\tilde{p_{\mathrm{T}}}$ the transverse momentum of the result of the recombination of $i$ and $j$, the two possible tags of a soft recombination are expressed as (i) $\min \left(p_{\mathrm{T}}^{i}, p_{\mathrm{T}}^{j}\right) / \tilde{p_{\mathrm{T}}}<0.1$ and (ii) $\Delta R_{i j}>m^{\text {orig }} / p_{\mathrm{T}}^{\text {orig }}$, with $m^{\text {orig }}$ and $p_{\mathrm{T}}^{\text {orig }}$ representing the mass and $p_{\mathrm{T}}$ of the original un-pruned $\mathrm{CA}$ jet. Soft recombinations are rejected, in which case the input four-vector with the smallest $p_{\mathrm{T}}$ is discarded and the input four-vector with the highest $p_{\mathrm{T}}$ is retained for further recombinations. A jet is considered as a $\mathrm{W}$-jet candidate if its pruned mass, $m_{\text {jet }}$, computed from the sum of the four-momenta of the 
constituents surviving the pruning, falls in the range $65<m_{\text {jet }}<105 \mathrm{GeV}$. Similarly, a Z-jet candidate is required to have $70<m_{\text {jet }}<110 \mathrm{GeV}$.

Further discrimination against jets from gluon and single-quark hadronization is obtained from the quantity called $N$-subjettiness [71]. The constituents of the jet before the pruning procedure are re-clustered with the $k_{\mathrm{T}}$ algorithm $[72,73]$, until $\mathrm{N}$ joint objects (subjets) remain in the iterative combination procedure of the $k_{\mathrm{T}}$ algorithm. The $\mathrm{N}$-subjettiness, $\tau_{N}$, is then defined as

$$
\tau_{N}=\frac{1}{d_{0}} \sum_{k} p_{\mathrm{T}, k} \min \left(\Delta R_{1, k}, \Delta R_{2, k}, \ldots, \Delta R_{N, k}\right),
$$

where the index $k$ runs over the PF constituents of the jet and the distances $\Delta R_{n, k}$ are calculated with respect to the axis of the $n$th subjet. The normalization factor $d_{0}$ is calculated as $d_{0}=\sum_{k} p_{\mathrm{T}, k} R_{0}$, setting $R_{0}$ to the jet radius of the original jet. The variable $\tau_{N}$ quantifies the capability of clustering the jet constituents in exactly $N$ subjets, with small values representing configurations more compatible with the $N$-subjets hypothesis. The ratio between 2 -subjettiness and 1-subjettiness, $\tau_{21}=\tau_{2} / \tau_{1}$, is found to be a powerful discriminant between jets originating from hadronic $\mathrm{V}$ decays and from gluon and singlequark hadronization. We reject $\mathrm{V}$-jet candidates with $\tau_{21}>0.75$. The remaining events are further categorized according to their value of $\tau_{21}$ in order to enhance the sensitivity of the analysis. Jets coming from hadronic $\mathrm{W}$ or $\mathrm{Z}$ decays in signal events are characterized by lower values of $\tau_{21}$ compared to the SM backgrounds.

\subsection{Final event selection and categorization}

After reconstructing the two vector bosons, we apply the final selections used for the search. In the $\ell \nu+\mathrm{V}$-jet $(\ell \ell+\mathrm{V}$-jet) channel, both the leptonic and the hadronic $\mathrm{V}$-boson candidates must have a $p_{\mathrm{T}}$ greater than $200(80) \mathrm{GeV}$. The larger threshold for the $\ell \nu+\mathrm{V}$-jet channel is related to the higher trigger thresholds and the larger multijet background in this category of events. In addition, there are specific topological selection criteria in the $\ell \nu+\mathrm{V}$ jet channel requiring that the two $\mathrm{W}$ bosons from the decay of a massive resonance are approximately back-to-back: the $\Delta R$ distance between the lepton and the $\mathrm{W}$-jet is greater than $\pi / 2$; the azimuthal angular separation between the missing transverse energy vector and the $\mathrm{W}$-jet is greater than 2.0 radians; and the azimuthal angular separation between the $\mathrm{W} \rightarrow \ell \nu$ and $\mathrm{W}$-jet candidates is greater than 2.0 radians. To further reduce the level of the $t \overline{\mathrm{t}}$ background in the $\ell \nu+\mathrm{V}$-jet channel, events are rejected if there is one or more b-tagged AK5 jet in the event, using a working point of the b-tagging algorithm tuned to provide a misidentification rate of $\sim 1 \%$ and efficiency of $\sim 70 \%$. This veto preserves about $90 \%$ of signal events. The looser selections in the $\ell \ell+\mathrm{V}$-jet channel allow the extension of the range of probed masses to lower values. The minimum value of $m_{\mathrm{VV}}$ is 700 (500) GeV for the $\ell \nu+\mathrm{V}$-jet $(\ell \ell+\mathrm{V}$-jet) channel, respectively.

To enhance the analysis sensitivity, we distinguish two V-jet categories:

- high-purity (HP) category: $\tau_{21} \leq 0.5$;

- low-purity (LP) category: $0.5<\tau_{21}<0.75$. 

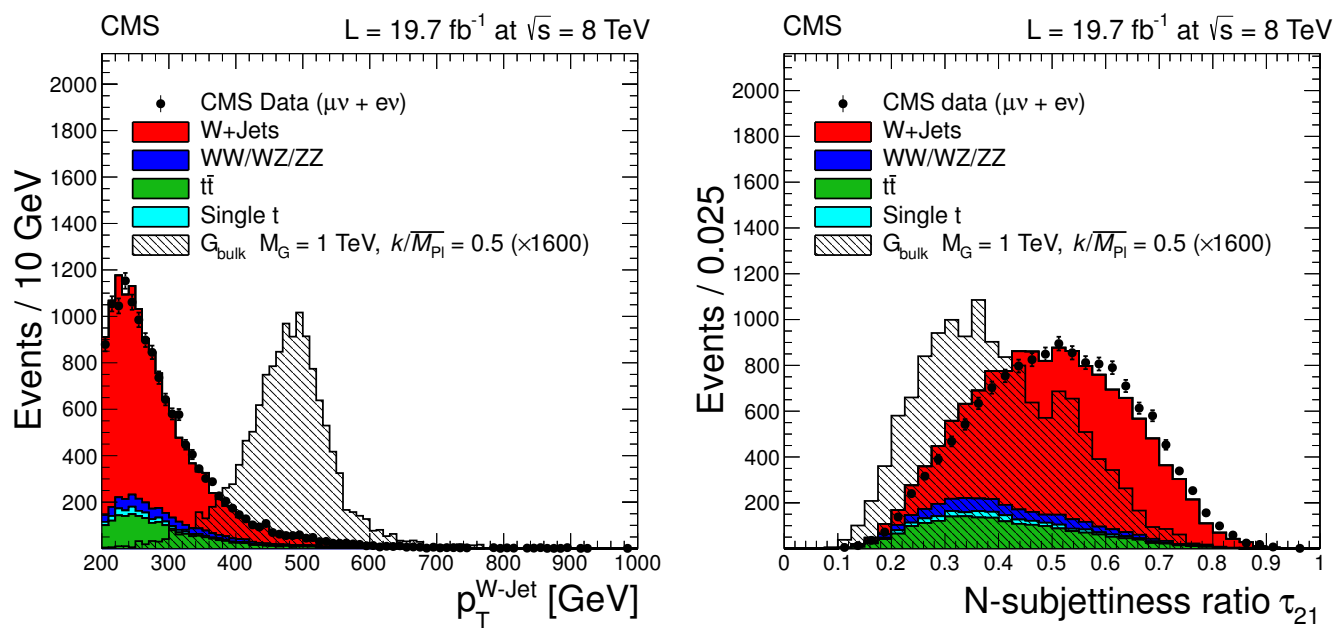

Figure 2. Hadronic $\mathrm{W} p_{\mathrm{T}}$ and $\mathrm{N}$-subjettiness ratio $\tau_{21}$ distributions for the combined muon and electron channels and with $65<m_{\text {jet }}<105 \mathrm{GeV}$. The VV, t $\overline{\mathrm{t}}$, and single-t backgrounds are taken from simulation and are normalized to the integrated luminosity of the data sample. The $\mathrm{W}+$ jets background is rescaled such that the total number of background events matches the number of events in data. The signal is scaled by a factor of 1600 for better visualization.

Although it is expected that the HP category dominates the total sensitivity of the analysis, the LP is retained, since for large masses of a new resonance it provides improved signal efficiency with only moderate background contamination. The final categorization is based on four classes of events, depending on their lepton flavor (muon or electron) and $\mathrm{V}$-jet purity (LP and HP). In case several distinct diboson resonance candidates are present in the same event, only one is kept for further analysis. Diboson pairs in the HP category are preferred to those in the LP category and, in case multiple choices are still possible, the candidate with the $\mathrm{V}$-jet with the highest $p_{\mathrm{T}}$ is retained. After the final selection no events with multiple leptonic $\mathrm{Z}$ candidates remain.

The $p_{\mathrm{T}}$ and $\tau_{21}$ distributions for the hadronic $\mathrm{W}(\mathrm{Z})$ boson candidate after the $\ell \nu+\mathrm{V}$-jet $\left(\ell \ell+\mathrm{V}\right.$-jet) selection are shown in figure 2 (figure 3), after applying a $65<m_{\text {jet }}<105 \mathrm{GeV}$ $\left(70<m_{\text {jet }}<110 \mathrm{GeV}\right)$ requirement. The $\tau_{21}$ distribution shows some disagreement between data and simulation. Previous studies suggest that part of this discrepancy can be attributed to a mismodeling of the parton showering in simulation [31]. The analysis is designed to be robust against differences between data and simulation independent of their specific sources, as described in the next sections.

\section{$5 \quad$ W tagging in a top-quark enriched control sample}

The data/simulation discrepancy observed in the key variable $\tau_{21}$ (figures 2 and 3 ) is of particular concern as the mismodeling of the variable could bias the signal efficiency estimated from the simulated samples. It is important to study the mismodeling in a signalfree sample with the characteristics of the jets similar to those expected for a genuine signal. In this way one can extract correction factors to apply to the signal efficiency suggested by the simulation and obtain a small systematic uncertainty related to this effect. A sample 

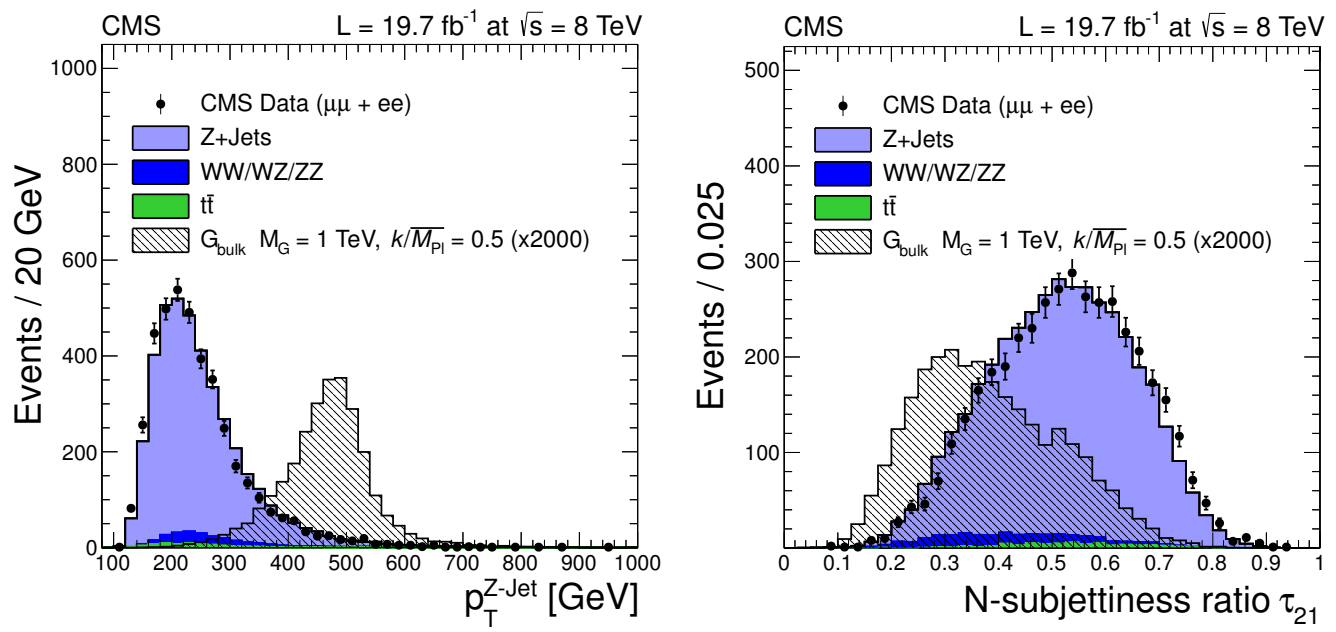

Figure 3. Hadronic $\mathrm{Z} p_{\mathrm{T}}$ and N-subjettiness ratio $\tau_{21}$ distributions for the combined muon and

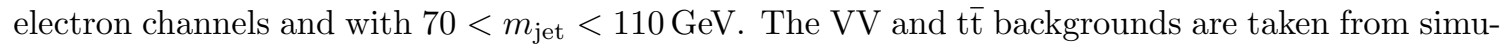
lation and are normalized to the integrated luminosity of the data sample. The $\mathrm{Z}+$ jets background is rescaled such that the total number of background events matches the number of events in data. The signal is scaled by a factor of 2000 for better visualization.

of high- $p_{\mathrm{T}} \mathrm{W}$ bosons decaying hadronically, and reconstructed as a single CA8 jet, can be isolated in $t \bar{t}$ and single top-quark events. The control sample is selected by applying all analysis requirements but inverting the b-jet veto. The data are compared with the predictions from simulation. Discrepancies between data and simulation are corrected in the analysis using the scale factors for top-quark background normalization, V-tagging efficiency, and peak and resolution of the $\mathrm{V}$-jet mass distribution derived in this section. Since the jet substructure produced in simulation depends on the modeling of the parton shower, PYTHIA v6.426 is used for this part of the event simulation. In this way the results of this study can be consistently applied to the signal MC samples, that are also generated with the same parton showering.

The $\tau_{21}$ distribution in the top-quark enriched control sample is shown in the left plot of figure 4, while the right plot shows the pruned jet mass distribution after applying the HP selection of $\tau_{21}<0.5$. The pruned jet mass plot shows a clear peak for events with an isolated $\mathrm{W}$ boson decaying to hadrons (W-signal component), as well as a combinatorial component mainly due to events where the extra $b$ jet from the top-quark decay is in the proximity of the W. From the comparison between data and simulation, a normalization correction factor for $\mathrm{t} \overline{\mathrm{t}}$ and single top-quark background processes is evaluated in the signal region $\left(65<m_{\text {jet }}<105 \mathrm{GeV}\right)$. The measured data-to-simulation scale factors are $0.97 \pm 0.02$ $(0.96 \pm 0.03)$ in the muon (electron) channel for the high-purity category, and $1.31 \pm 0.05$ $(1.39 \pm 0.08)$ for the low-purity category. These scale factors (including both the W-signal and the combinatorial components) are used to correct the normalization of the $t \bar{t}$ and single top-quark simulated background predictions in the signal region.

A simultaneous fit to the jet mass distributions, before and after the $m_{\text {jet }}$ and $\tau_{21}$ requirements, is performed to separate the $\mathrm{W}$-signal from the combinatorial components in 

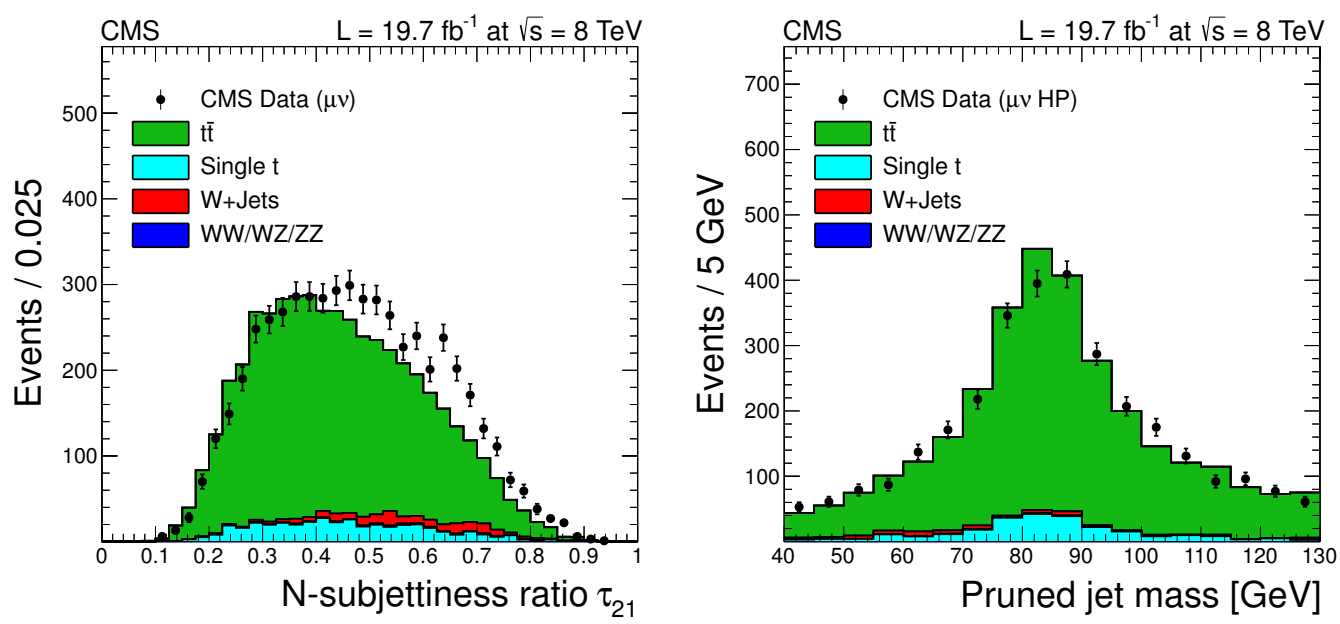

Figure 4. Distributions from the top-quark enriched control sample in the muon channel. Left: $\mathrm{N}$-subjettiness ratio $\tau_{21}$, Right: $m_{\text {jet }}$ after requiring $\tau_{21}<0.5$. The distributions show some disagreement between data and simulation. The simulation is corrected for these discrepancies using the method based on data described in section 5 . This approach ensures that the analysis is robust against differences between data and simulation, independent of their sources.

the top-quark enriched sample, in both data and simulation. The fit results are used to extract the efficiencies for identifying an isolated hadronic $\mathrm{W}$ boson (W tagging based on $m_{\text {jet }}$ and $\tau_{21}$ requirements). Differences in the resulting $\mathrm{W}$-tagging efficiencies will be driven by the discrepancy between data and simulation in the $\tau_{21}$ distribution. The ratio of the efficiency in data and simulation yields W-tagging scale factors that are used to correct the total signal efficiency predicted by the simulation. The scale factor for $\mathrm{W}$ tagging is $0.89 \pm 0.08(1.28 \pm 0.30)$ for the high-purity (low-purity) category, combining the muon and electron channels.

In addition, the $\mathrm{W}$-jet mass peak position and resolution are extracted from the same fit and are measured to be $83.4 \pm 0.3$ and $7.2 \pm 0.4 \mathrm{GeV}$, respectively, in the simulation and $84.7 \pm 0.4$ and $7.9 \pm 0.6 \mathrm{GeV}$ in the data, where the uncertainties given are statistical only. The mass peak position is slightly shifted with respect to the $\mathrm{W}$-boson mass because of the presence of extra energy deposited in the jet cone coming from pileup, underlying event, and initial-state radiation not completely removed by the jet pruning procedure. For events with top quarks, additional energy contributions come also from the possible presence of a $\mathrm{b}$ jet close to the $\mathrm{W}$-jet candidate.

The same corrections are used also in the case where the $\mathrm{V}$-jet is assumed to come from a $\mathrm{Z}$ boson. The kinematic properties of $\mathrm{W}$-jets and Z-jets are very similar and the agreement between data and simulation is expected to be equally good.

\section{Modeling of background and signal}

\subsection{Background estimation}

After the full selection, the dominant background comes from SM V+jets events. A procedure based on data has been developed in order to estimate this background. Other minor 


\begin{tabular}{|ccccc|}
\hline & $\mu \nu+\mathrm{V}$-jet HP & $\mu \nu+\mathrm{V}$-jet LP & e $\nu+$ V-jet HP & e $\nu+$ V-jet LP \\
\hline Observed yield & 1483 & 1546 & 892 & 988 \\
Expected background & $1434 \pm 38$ & $1644 \pm 41$ & $878 \pm 30$ & $978 \pm 31$ \\
\hline Bulk graviton $\left(k / \bar{M}_{\mathrm{Pl}}=0.5\right)$ & \multicolumn{4}{c}{ Signal expectation $(\mathrm{MC})$} \\
\hline$m_{\mathrm{G}}=800 \mathrm{GeV}$ & 12.8 & 5.1 & 10.1 & 3.9 \\
$m_{\mathrm{G}}=1200 \mathrm{GeV}$ & 0.92 & 0.43 & 0.79 & 0.37 \\
\hline
\end{tabular}

Table 1. Observed and expected yields for the $\ell \nu+V$-jet analysis. The yields are quoted in the range $700<m_{\mathrm{WW}}<3000 \mathrm{GeV}$. The expected background is quoted from the sideband procedure. The uncertainties in the background prediction from data are statistical in nature, as they depend on the number of events in the sideband region. Statistical uncertainties in the signal expectations are negligible.

\begin{tabular}{|ccccc|}
\hline & $\mu \mu+\mathrm{V}$-jet HP & $\mu \mu+\mathrm{V}$-jet LP & ee + V-jet HP & ee + V-jet LP \\
\hline Observed yield & 575 & 338 & 360 & 233 \\
Expected background & $622 \pm 29$ & $338 \pm 22$ & $370 \pm 22$ & $207 \pm 17$ \\
\hline Bulk graviton $\left(k / \bar{M}_{\mathrm{Pl}}=0.5\right)$ & \multicolumn{4}{c}{ Signal expectation $(\mathrm{MC})$} \\
\hline$m_{\mathrm{G}}=800 \mathrm{GeV}$ & 2.4 & 0.5 & 2.0 & 0.4 \\
$m_{\mathrm{G}}=1200 \mathrm{GeV}$ & 0.16 & 0.04 & 0.14 & 0.035 \\
\hline
\end{tabular}

Table 2. Observed and expected yields for the $\ell \ell+\mathrm{V}$-jet analysis. The yields are quoted in the range $500(650)<m_{\mathrm{ZZ}}<2800 \mathrm{GeV}$ for the HP (LP) category. The expected background is quoted from the sideband procedure. The uncertainties in the background predictions from data are statistical in nature, as they depend on the number of events in the sideband region. Statistical uncertainties in the signal expectations are negligible.

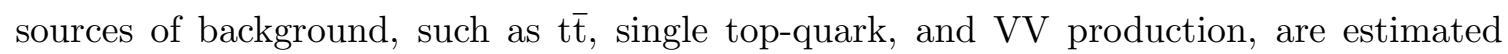
using the simulated samples after applying correction factors based on control samples in data, as described in the previous sections. A signal-depleted control region is defined around the $m_{\text {jet }}$ mass window described in section 4.6. For the $\ell \nu+\mathrm{V}$-jet channel, lower and upper sideband regions are defined in the $m_{\text {jet }}$ ranges $[40,65]$ and $[105,130] \mathrm{GeV}$, respectively. In the $\ell \ell+\mathrm{V}$-jet channel, the sidebands are defined in the $m_{\text {jet }}$ ranges $[50,70]$ and $[110,130] \mathrm{GeV}$.

The overall normalization of the $\mathrm{V}+$ jets background in the signal region is determined from a fit to the $m_{\text {jet }}$ distribution in the lower and upper sidebands of the observed data. The analytical form of the fit function is chosen from simulation studies and the minor backgrounds are taken from the simulation. Figures 5 and 6 show the result of this fit procedure for the $\ell \nu+\mathrm{V}$-jet and $\ell \ell+\mathrm{V}$-jet analyses, respectively. Tables 1 and 2 show the predicted number of background events in the signal region after the inclusion of the minor backgrounds and compare it with the data.

The shape of the $m_{\mathrm{VV}}$ distribution of the $\mathrm{V}+$ jets background in the signal region is determined from the low $m_{\text {jet }}$ sideband only, through an extrapolation function $\alpha_{\mathrm{MC}}\left(m_{\mathrm{VV}}\right)$ 

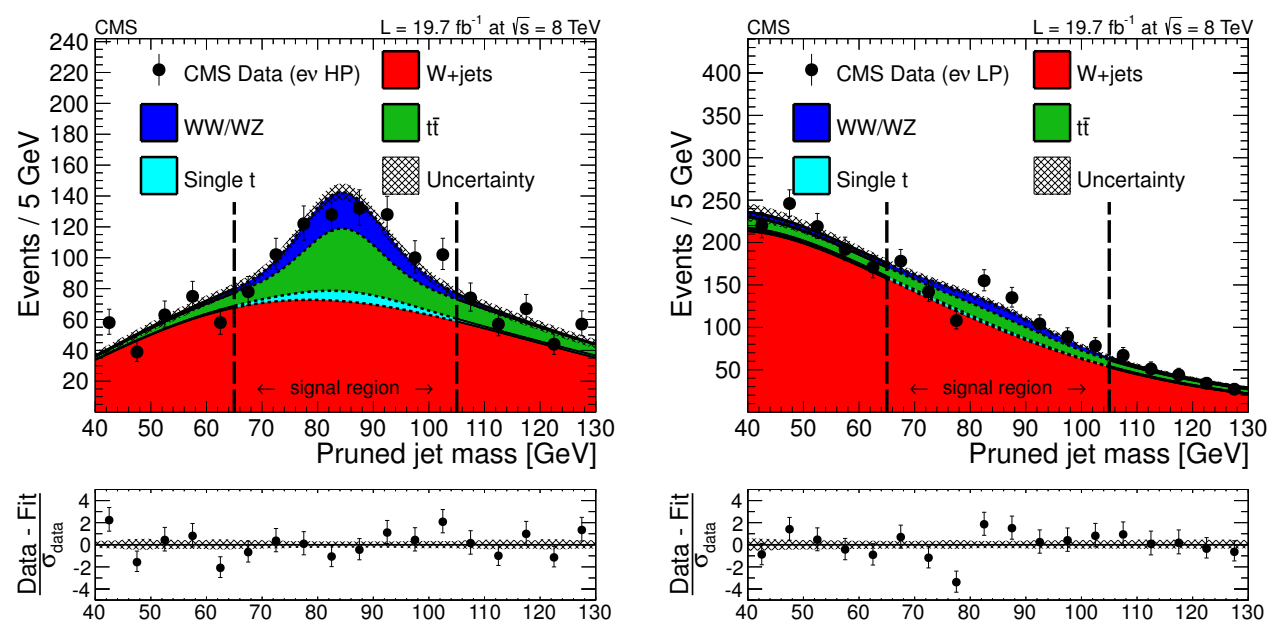

Figure 5. Distributions of the pruned jet mass, $m_{\text {jet }}$, in the $\ell \nu+V$-jet analysis in the electron channel. The left (right) panel shows the distribution for the HP (LP) category. All selections are applied except the final $m_{\text {jet }}$ signal window requirement. Data are shown as black markers. The prediction of the non-resonant $\mathrm{W}+$ jets background comes from a fit excluding the signal region (between the vertical dashed lines), while the predictions for the minor backgrounds come from the simulation. The MC resonant shapes are corrected using the differences between data and simulation in the $\mathrm{W}$ peak position and width measured in the $t \overline{\mathrm{t}}$ control region (see section 5 ). At the bottom of each plot, the bin-by-bin fit residuals, (data-fit) $/ \sigma_{\text {data }}$, are shown together with the uncertainty band of the fit normalized by $\sigma_{\text {data }}$.
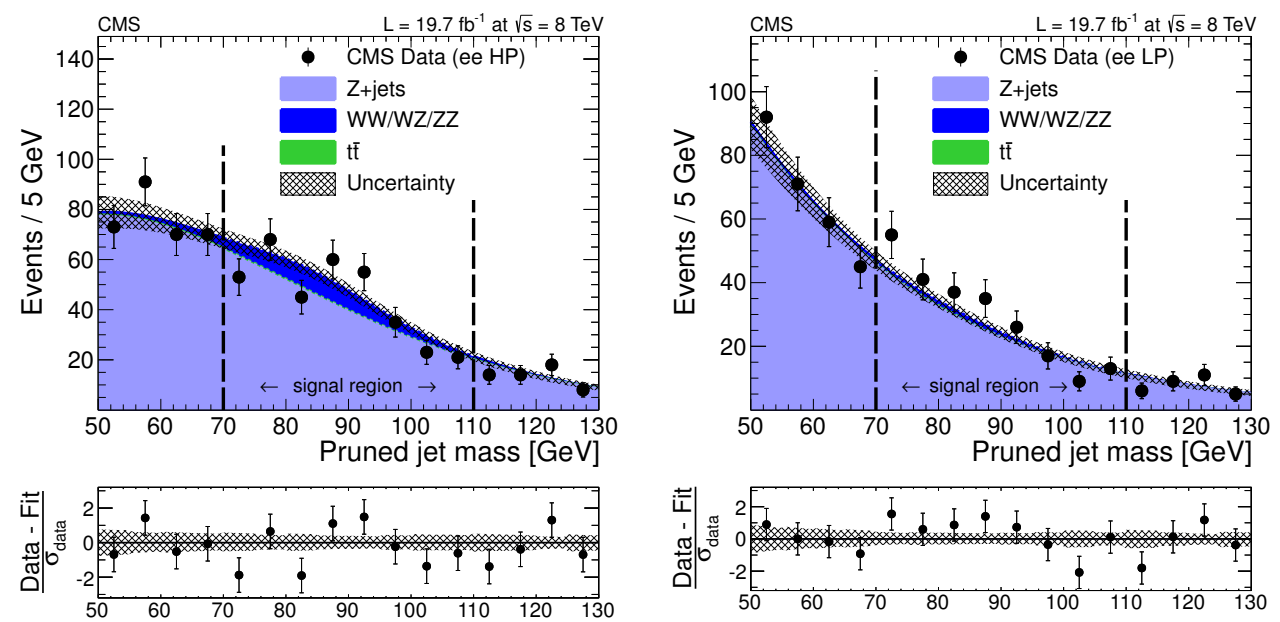

Figure 6. Distributions of the pruned jet mass, $m_{\text {jet }}$, in the $\ell \ell+V-j e t$ analysis in the electron channel. The left (right) panel shows the distribution for the HP (LP) category. All selections are applied except the final $m_{\text {jet }}$ signal window requirement. Data are shown as black markers. The prediction of the non-resonant $\mathrm{Z}+$ jets background comes from a fit excluding the signal region (between the vertical dashed lines), while the predictions for the minor backgrounds come from the simulation. The MC resonant shapes are corrected using the differences between data and simulation in the $\mathrm{W}$ peak position and width measured in the $t \overline{\mathrm{t}}$ control region (see section 5 ). At the bottom of each plot, the bin-by-bin fit residuals, (data-fit) $/ \sigma_{\text {data }}$, are shown together with the uncertainty band of the fit normalized by $\sigma_{\text {data }}$. 
derived from the $\mathrm{V}+$ jets simulation, defined as:

$$
\alpha_{\mathrm{MC}}\left(m_{\mathrm{VV}}\right)=\frac{F_{\mathrm{MC}, \mathrm{SR}}^{\mathrm{V}+\text { jets }}\left(m_{\mathrm{VV}}\right)}{F_{\mathrm{MC}, \mathrm{SB}}^{\mathrm{V}+\text { jets }}\left(m_{\mathrm{VV}}\right)},
$$

where $F_{\mathrm{MC}, \mathrm{SR}}^{\mathrm{V}+\text { jets }}\left(m_{\mathrm{VV}}\right)$ and $F_{\mathrm{MC}, \mathrm{SB}}^{\mathrm{V}+\text { jets }}\left(m_{\mathrm{VV}}\right)$ are the probability density functions used to describe the $m_{\mathrm{VV}}$ spectrum in simulation for the signal region and low $m_{\text {jet }}$ sideband region, respectively. The high $m_{\text {jet }}$ sideband was not considered in order to exclude possible contamination from beyond-SM resonances decaying into a V boson and a SM Higgs boson, H, with mass of $125.6 \mathrm{GeV}$ [74], in addition to the VV final state considered here. The partial compositeness model [14] is an example of such a scenarios. These signal events from HV resonances, in which the Higgs boson is reconstructed as a jet in the CMS detector and the $\mathrm{V}$ decays leptonically, would populate the high-mass sideband region of both the $\ell \nu+\mathrm{V}$-jet $\left(m_{\text {jet }} \in[105,130] \mathrm{GeV}\right)$ and $\ell \ell+\mathrm{V}$-jet $\left(m_{\text {jet }} \in[110,130] \mathrm{GeV}\right)$ analyses. This possibility cannot be ignored because this search is not limited only to the bulk graviton model but includes also a model-independent interpretation of the results (section 8.2).

The $m_{\mathrm{VV}}$ distribution observed in the lower sideband region is corrected for the presence of minor backgrounds in order to have an estimation of the $\mathrm{V}+$ jets contribution in the control region of the data, $F_{\mathrm{DATA}, \mathrm{SB}}^{\mathrm{V}+\text { jets }}\left(m_{\mathrm{VV}}\right)$. The shape of the $\mathrm{V}+$ jets background distribution in the signal region is obtained by rescaling $F_{\mathrm{DATA}, \mathrm{SB}}^{\mathrm{V}+\text { jets }}\left(m_{\mathrm{VV}}\right)$ for $\alpha_{\mathrm{MC}}\left(m_{\mathrm{VV}}\right)$. The final prediction of the background contribution in the signal region, $N_{\mathrm{SR}}^{\mathrm{BKGD}}\left(m_{\mathrm{VV}}\right)$, is given by

$$
N_{\mathrm{SR}}^{\mathrm{BKGD}}\left(m_{\mathrm{VV}}\right)=C_{\mathrm{SR}}^{\mathrm{V}+\text { jets }} \times F_{\mathrm{DATA}, \mathrm{SB}}^{\mathrm{V}+\text { jets }}\left(m_{\mathrm{VV}}\right) \times \alpha_{\mathrm{MC}}\left(m_{\mathrm{VV}}\right)+\sum_{k} C_{\mathrm{SR}}^{k} F_{\mathrm{MC}, \mathrm{SR}}^{k}\left(m_{\mathrm{VV}}\right),
$$

where the index $k$ runs over the list of minor backgrounds and $C_{\mathrm{SR}}^{\mathrm{V}+\text { jets }}$ and $C_{\mathrm{SR}}^{k}$ represent the normalizations of the yields of the dominant $\mathrm{V}+$ jets background and of the different minor background contributions. The ratio $\alpha_{\mathrm{MC}}\left(m_{\mathrm{VV}}\right)$ reflects small kinematic differences between the signal region and sideband, which are mostly independent from the theoretical prediction of cross sections. To test the validity and the robustness of the method, a closure test with data has been performed, predicting successfully the normalization and shape of the $\mathrm{V}+$ jets background in an upper sideband using the lower sideband data. The $m_{\mathrm{VV}}$ distribution of the background in the signal region is described analytically by a function defined as $f(x) \propto \mathrm{e}^{-x /\left(c_{0}+c_{1} x\right)}$. Alternative fit functions have been studied but their usage does not change the final performance. The $m_{\mathrm{VV}}$ range of the fit determines the region of masses probed by the searches. The range has been chosen such that there is a smoothly falling spectrum, in order to have a stable fit and robust control of the background estimation. For the $\ell \nu+\mathrm{V}$-jet analysis, the fits are carried out in the $m_{\mathrm{VV}}$ range $[700,3000] \mathrm{GeV}$. In the $\ell \ell+\mathrm{V}$-jet analysis, the ranges for the HP and LP categories are $[500,2800]$ and $[650,2800] \mathrm{GeV}$, respectively. The fits are always unbinned. In the $\ell \ell+\mathrm{V}-$ jet analysis, the shapes of the background distributions for the muon and electron channels are found to be statistically compatible. The final shape estimation for the $\ell \ell+\mathrm{V}$ jet analysis has been carried out integrating over the two lepton flavors in order to reduce the statistical uncertainties in the fitted parameters. 

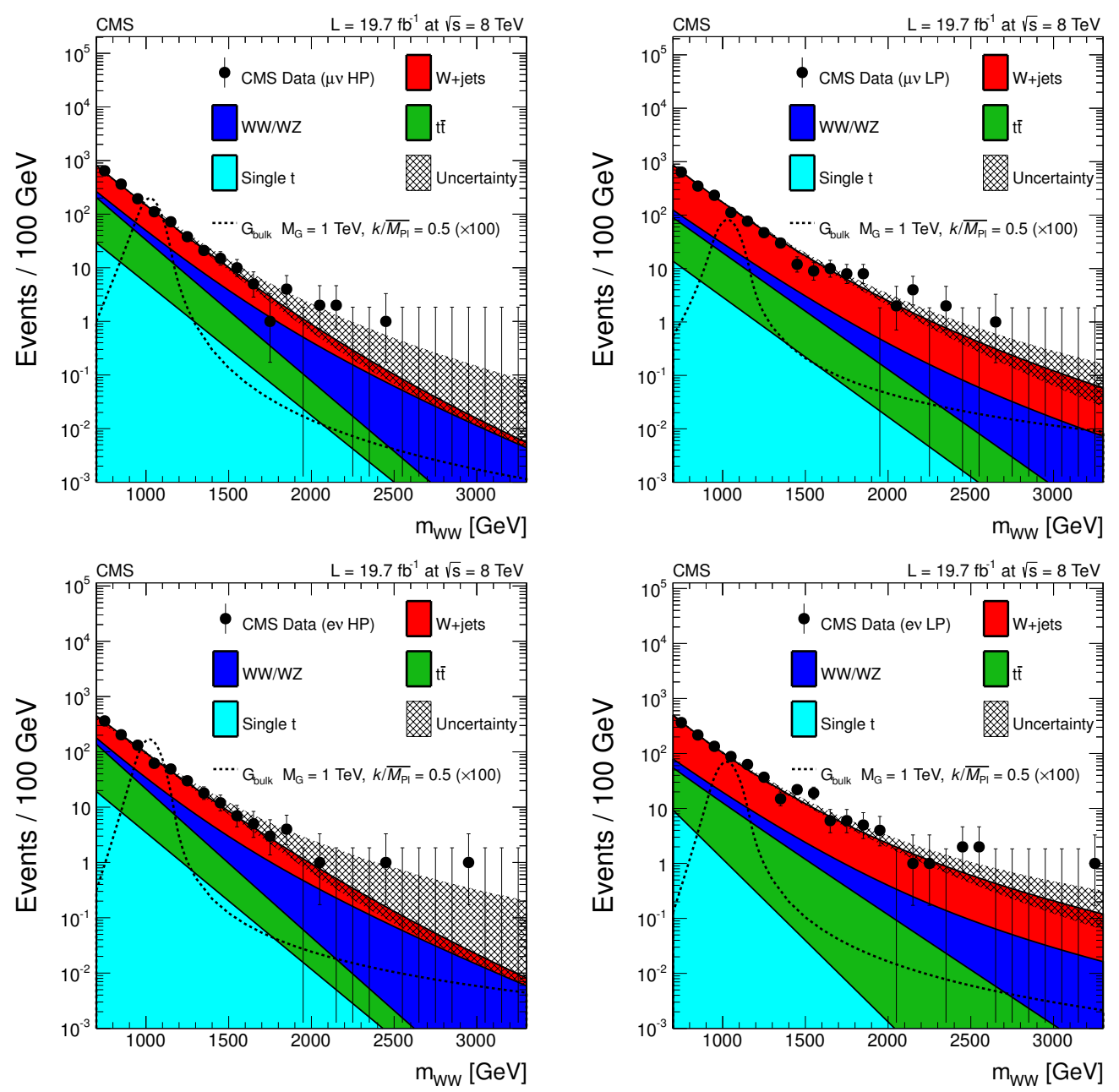

Figure 7. Final distributions in $m_{\mathrm{WW}}$ for data and expected backgrounds for both the muon (top) and the electron (bottom) channels, high-purity (left) and low-purity (right) categories. The $68 \%$ error bars for Poisson event counts are obtained from the Neyman construction as described in ref. [75]. Also shown is a hypothetical bulk graviton signal with mass of $1000 \mathrm{GeV}$ and $k / \bar{M}_{\mathrm{Pl}}=0.5$. The normalization of the signal distribution is scaled up by a factor of 100 for a better visualization.

Figure 7 shows the final observed spectrum in $m_{\mathrm{WW}}$ of the selected events in the four categories of the $\ell \nu+\mathrm{V}$-jet analysis. The observed data and the predicted background agree with each other. The highest-mass event in the $\ell \nu+\mathrm{V}$-jet channel is from the electron LP category and it has $m_{\mathrm{WW}} \approx 3200 \mathrm{GeV}$. This event is not included in the statistical analysis of section 8 , which is performed up to $m_{\mathrm{WW}}$ of $3 \mathrm{TeV}$. The impact of this event on the reported results is negligible since we produce limits for a narrow bulk graviton with a resonance mass up to $2.5 \mathrm{TeV}$. The observed event is compatible at the $1 \sigma$ level with the background prediction for $m_{\mathrm{WW}}$ above $2.5 \mathrm{TeV}$.

The $m_{\mathrm{ZZ}}$ distribution of the selected events in the $\ell \ell+\mathrm{V}$-jet analysis is presented in figure 8. Also in this case, an overall good description in both normalization and shape of the data by the background estimation is observed. The highest mass event in the $\ell \ell+V$-jet channel is from the electron $\mathrm{HP}$ category and it has $m_{\mathrm{ZZ}} \approx 2600 \mathrm{GeV}$. 

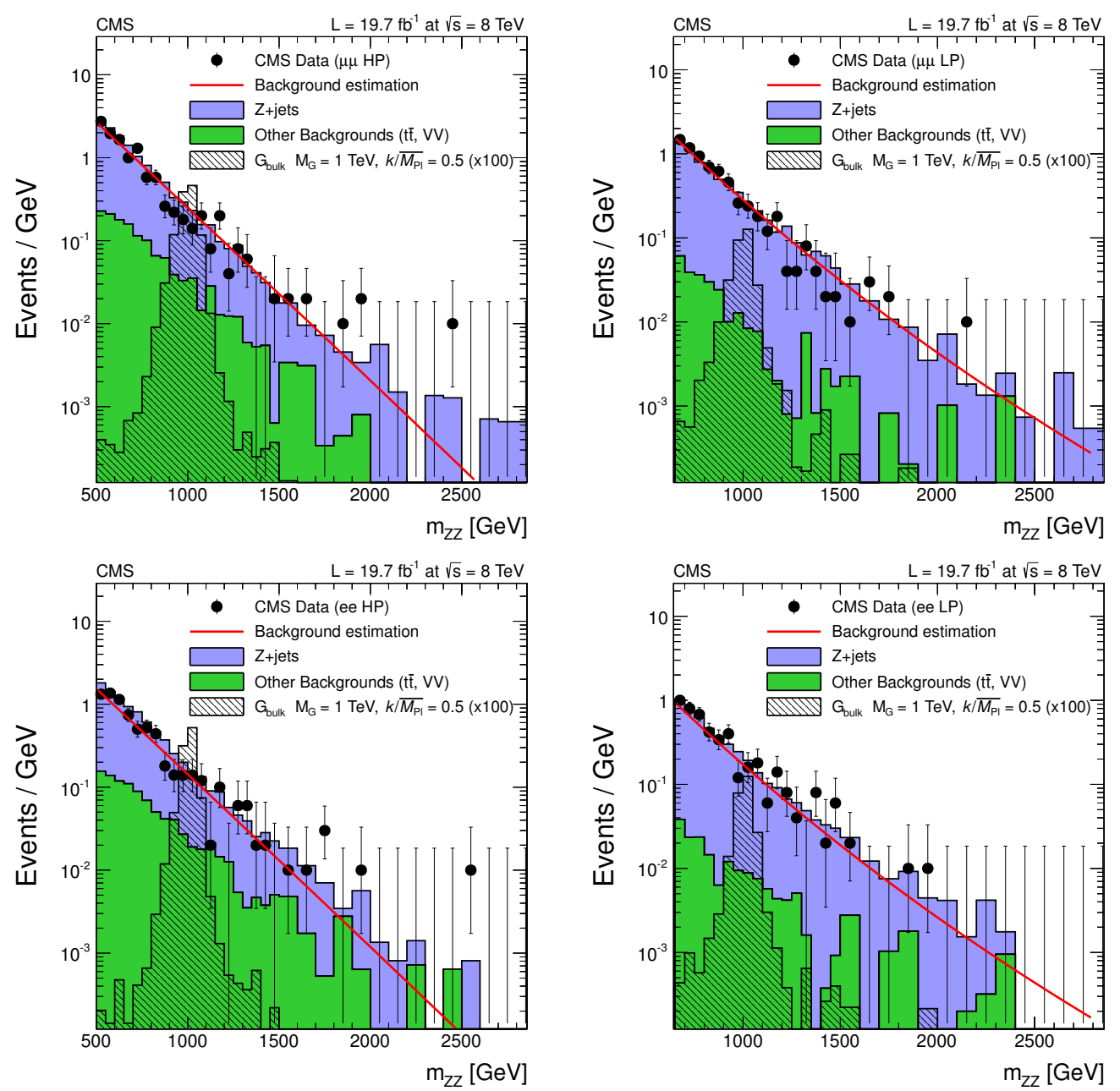

Figure 8. Final distributions in $m_{\mathrm{ZZ}}$ for data and expected backgrounds for both the muon (top) and the electron (bottom) channels, high-purity (left) and low-purity (right) categories. Points with error bars show distributions of data; solid histograms depict the different components of the background expectation from simulated events. The $68 \%$ error bars for Poisson event counts are obtained from the Neyman construction as described in ref. [75]. Also shown is a hypothetical bulk graviton signal with mass of $1000 \mathrm{GeV}$ and $k / \bar{M}_{\mathrm{Pl}}=0.5$. The normalization of the signal distribution is scaled up by a factor of 100 for a better visualization. The solid line shows the central value of the background predicted from the sideband extrapolation procedure.

\subsection{Modeling of the signal mass distribution}

The shape of the reconstructed signal mass distribution is extracted from the bulk graviton $\mathrm{MC}$ samples generated with the coupling $k / \bar{M}_{\mathrm{Pl}}=0.2$, corresponding to an intrinsic relative width of the resonance of about $0.2 \%$. For models with $k / \bar{M}_{\mathrm{Pl}} \lesssim 0.5$, the natural width of the resonance is sufficiently small to be neglected when compared to the detector resolution. This makes our modeling of the detector effects on the signal shape independent of the actual model used for generating the events. In the final analysis of the $m_{\mathrm{VV}}$ spectrum, the discovery potential and exclusion power both depend on an accurate description of the signal shape. We adopt an analytical description of the signal shape, 
choosing a double-sided Crystal-Ball (CB) function (i.e., a Gaussian core with power law tails on both sides) [76] to describe the CMS detector resolution. To take into account differences between muon and electron $p_{\mathrm{T}}$ resolutions at high $p_{\mathrm{T}}$, the signal mass distribution is parametrized separately for events with electrons and muons. No appreciable differences have been observed in the $m_{\mathrm{VV}}$ signal shape between low- and high-purity categories. The typical width of the Gaussian core is about $3 \%-5 \%$ of the nominal mass in the $\ell \ell+V$-jet channel or $4 \%-6 \%$ for the $\ell \nu+V$-jet channel.

\section{Systematic uncertainties}

\subsection{Systematic uncertainties in the background estimation}

Uncertainties in the estimation of the background affect both the normalization and shape of the $m_{\mathrm{VV}}$ distribution. Uncertainties in the background normalization are mainly statistical in nature and scale with the amount of data in the sideband regions and the number of events in the simulated samples. Tables 1 and 2 show the uncertainties in the background expectations for the $\ell \nu+\mathrm{V}$-jet and $\ell \ell+\mathrm{V}$-jet analyses, respectively. The systematic uncertainties in the $\mathrm{V}+$ jets background normalization are dominated by the statistical uncertainty associated with the number of events in data in the $m_{\text {jet }}$ sideband regions (below $10 \%)$. The systematic uncertainty in the $t \bar{t}$ normalization comes from the uncertainties in the data-to-simulation scale factors derived in the top-quark enriched control sample (below 5\%). The systematic uncertainty in the WW inclusive cross section is assigned to be $20 \%$, taken from the relative difference in the mean value between the published CMS cross section measurement at $\sqrt{s}=8 \mathrm{TeV}$ and the SM expectation [77]. An additional systematic uncertainty in the WW normalization comes from the uncertainty in the V-tagging scale factors derived in section 5. The same uncertainties derived for WW are also used for WZ and ZZ processes.

Systematic uncertainties in the background shape are estimated from the covariance matrix of the fit to the extrapolated sidebands and from the uncertainties in the modeling of $\alpha_{\mathrm{MC}}\left(m_{\mathrm{VV}}\right)$. They are both statistical in nature, as they are driven by the available data in the sidebands and the number of events generated for the simulation of the $\mathrm{V}+$ jets background.

\subsection{Systematic uncertainties in the signal prediction}

Systematic uncertainties affect both the signal efficiency and the $m_{\mathrm{VV}}$ shape. Table 3 presents the primary uncertainties in the signal normalization. Among the sources of systematic uncertainty in the signal efficiency are the muon momentum scale and resolution, the electron energy scale and resolution, the jet energy scale and resolution, and the unclustered energy in the event. The event selection is applied to signal samples after varying the lepton four-momenta within one standard deviation of the corresponding uncertainty in the muon momentum scale [54] or electron energy scale [56], or applying an appropriate momentum/energy smearing in case of resolution uncertainties. The same procedure is also applied for the jet four-momenta using the corresponding energy scale and resolution 
uncertainties [64]. In this process, variations in the lepton and jet four-momenta are propagated consistently to the $E_{\mathrm{T}}^{\text {miss }}$ vector. The signal efficiency is then recalculated using modified lepton and jet four-momenta separately for each source of systematic uncertainties. The largest relative change in the signal efficiency compared to the default value is taken as the systematic uncertainty for that specific source. The muon, electron, and jet uncertainties are assumed to be uncorrelated.

The systematic uncertainties in the lepton trigger, identification, and isolation efficiencies are derived using a dedicated tag-and-probe analysis in $\mathrm{Z} \rightarrow \ell^{+} \ell^{-}$events. The uncertainties in trigger and identification+isolation efficiencies for muons are $3 \%$ and $4 \%$, respectively. The total uncertainty in the electron trigger, identification, and isolation efficiency is $3 \%$. These uncertainties are evaluated taking into account the limited number of data events in the boosted regime. We also include systematic uncertainties in signal efficiency due to uncertainties in data-to-simulation scale factors for the V-tagging identification (derived from the top-quark enriched control sample, see section 5), and b-jet identification efficiencies (derived with the methods described in ref. [63] and updated with the $8 \mathrm{TeV}$ data). The systematic uncertainties from pileup are assigned by re-weighting the signal simulation samples such that the distribution of the number of interactions per bunch crossing is shifted up and down by one standard deviation compared with that found in data [78]. The impact of these changes on the signal efficiency is used to assess the systematic effect. The impact of the proton PDF uncertainties on the signal efficiency is evaluated with the PDF4LHC prescription [79] using MSTW [80] and NNPDF [81] PDF sets. The uncertainty in the integrated luminosity is $2.6 \%$ [82].

Uncertainties in the scale and resolution of the four-momenta of the reconstructed objects can bias the peak and smear the width of the signal profile. The systematic uncertainties considered to affect the signal shape are the scale and resolution uncertainties on muons, electrons, jets, and the unclustered energy scale. For each of these sources of experimental uncertainty, the lepton/jets four-momenta and $E_{\mathrm{T}}^{\text {miss }}$ vector are varied (or smeared) by the relative uncertainty. In general, only small effects on the peak position and the width of the Gaussian core of the signal shapes have been found. The jet energy scale and resolution introduce a relative uncertainty of about $3 \%(2 \%)$ in the signal width for the $\ell \nu+V$-jet $(\ell \ell+V$-jet $)$ channel. In the $\ell \nu+V$-jet channel, the unclustered energy scale introduces a 1-3\% uncertainty in the signal width, larger at low resonance masses. In the $\ell \ell+\mathrm{V}$-jet channel, the muon resolution causes an additional relative uncertainty of $2 \%$ in the signal width. The uncertainty in the peak position of the signal is estimated to be less than $1 \%$.

\section{Statistical interpretation}

The comparison between the $m_{\mathrm{VV}}$ distribution observed in data and the background prediction from data is used to test for the presence of a resonance decaying to vector bosons. We set upper limits on the production cross section of a new resonance decaying to the WW final state or the ZZ final state by combining the four event categories of the $\ell \nu+V$-jet analysis or the $\ell \ell+\mathrm{V}$-jet analysis, respectively. We follow the modified frequentist prescrip- 


\begin{tabular}{|lcc|}
\hline Source & \multicolumn{2}{c|}{ Analysis } \\
& $\ell \nu+\mathrm{V}$-jet & $\ell \ell+\mathrm{V}$-jet \\
\hline Muons (trigger and ID) & $2 \%$ & $5 \%$ \\
Muon scale & $1 \%$ & $2 \%$ \\
Muon resolution & $<0.1 \%$ & $0.5 \%$ \\
Electrons (trigger and ID) & $3 \%$ & $3 \%$ \\
Electron scale & $<0.5 \%$ & $<0.5 \%$ \\
Electron resolution & $<0.1 \%$ & $<0.1 \%$ \\
Jet scale & $1-3 \%$ & $1 \%$ \\
Jet resolution & $<0.5 \%$ & $<0.1 \%$ \\
Unclustered energy scale & $<0.5 \%$ & - \\
Pileup & $0.5 \%$ & $0.5 \%$ \\
V tagging & \multicolumn{2}{c|}{$9 \%(\mathrm{HP})$} \\
& $24 \%(\mathrm{LP})$ \\
PDF & \multicolumn{2}{c}{$<0.5 \%$} \\
Luminosity & \multicolumn{2}{c}{$2.6 \%$} \\
\hline
\end{tabular}

Table 3. Summary of systematic uncertainties in signal yield, relative to the expected number of observed signal events. All systematic uncertainties in the list are treated as uncorrelated.

tion described in refs. $[83,84]\left(\mathrm{CL}_{S}\right.$ method). The limits are computed using an unbinned shape analysis. Systematic uncertainties are treated as nuisance parameters and profiled in the statistical interpretation using log-normal priors.

\subsection{Limits on a narrow-width bulk graviton model}

Exclusion limits can be set in the context of the bulk graviton model, under the assumption of a natural width negligible with respect to the experimental resolution (narrow-width approximation). Figure 9 shows the $95 \%$ confidence level (CL) expected and observed exclusion limits as a function of $\mathrm{M}_{\mathrm{G}}$. The limits are compared with the cross section times the branching fraction to $\mathrm{WW}$ and $\mathrm{ZZ}$ for a bulk graviton with $k / \bar{M}_{\mathrm{Pl}}=0.2$ and $k / \bar{M}_{\mathrm{Pl}}=0.5$. These results were cross-checked with an alternative background estimation from data, fitting the $m_{\mathrm{VV}}$ distributions for the same selected events with a smoothly falling function. This approach, common to previously released CMS results [19, 32, 85], provides results very close to the baseline method described above, further strengthening our confidence in the robustness of the background estimation method.

The $\ell \nu+V$-jet and $\ell \ell+V$-jet analyses are further combined together with a complementary CMS search in the $\mathrm{VV} \rightarrow\left(\mathrm{q} \overline{\mathrm{q}}^{(\prime)}\right)\left(\mathrm{q} \overline{\mathrm{q}}^{(\prime)}\right) \rightarrow 2$ V-jets final state [32] (dijet channel), in order to maximize the sensitivity of the search for this specific model. The fully hadronic analysis uses the same techniques to identify V-jets discussed in section 4.6. The systematic uncertainties in jet energy scale/resolution, V-tagging scale factors, and luminosity 

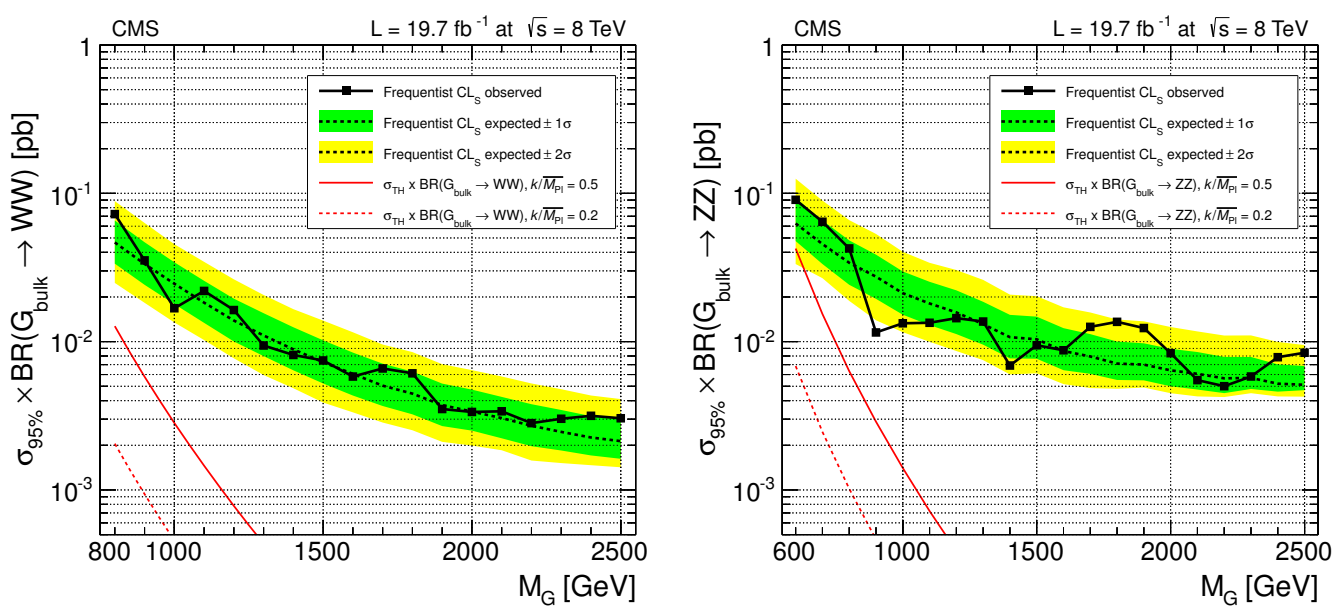

Figure 9. Observed (solid) and expected (dashed) 95\% CL upper limits on the product of the graviton production cross section and the branching fraction of $\mathrm{G}_{\text {bulk }} \rightarrow \mathrm{WW}$ (left) and $\mathrm{G}_{\text {bulk }} \rightarrow \mathrm{ZZ}$ (right). The cross section for the production of a bulk graviton multiplied by its branching fraction for the relevant process is shown as a red solid (dashed) curve for $k / \bar{M}_{\mathrm{Pl}}=0.5(0.2)$, respectively.

are considered correlated at $100 \%$ among the three analyses entering the statistical combination. The systematic uncertainties in electrons and muons are considered correlated at $100 \%$ between the $\ell \ell+\mathrm{V}$-jet and $\ell \nu+\mathrm{V}$-jet channels. The resulting $95 \% \mathrm{CL}$ upper limits on the signal cross section are shown in figure 10 . The $\ell \ell+\mathrm{V}$-jet channel is the only one contributing to the limit for resonance masses below $800 \mathrm{GeV}$. In the range $800-2500 \mathrm{GeV}$, the $\ell \nu+\mathrm{V}$-jet channel dominates the sensitivity, although the $\ell \ell+\mathrm{V}$-jet and dijet channels give significant contributions to the combined limit in the region below and above $1300 \mathrm{GeV}$, respectively. Because of the combination of the analyses, the expected upper limits on cross section are made more stringent by about $15-20 \%$ compared to the individual $\ell \nu+\mathrm{V}$-jet channel, depending on the resonance mass. The integrated luminosity of the sample is not large enough to allow us to set mass limits on the bulk graviton models with $k / \bar{M}_{\mathrm{Pl}}=0.2$ or 0.5. Figure 10 (right) presents also the local p-value of the significance of the excesses observed in the data. No excesses with significances larger than two standard deviations are observed.

\subsection{Model-independent limits}

The analysis as presented above is specific to the case of a narrow bulk graviton model, but this is not the only extension of the SM predicting resonances decaying to vector bosons. Therefore it is useful to allow the reinterpretation of these results in a generic model. In this section we present the exclusion limits on the visible number of events after having introduced some modifications to the analysis that greatly simplify its structure, at a moderate price in terms of performance. Together with the upper limits on the number of signal events, we provide tables with the reconstruction and identification efficiencies for vector bosons in the kinematic acceptance of the analysis. Following the instructions detailed in appendix A, it is possible to estimate the number of events for a generic signal model that 

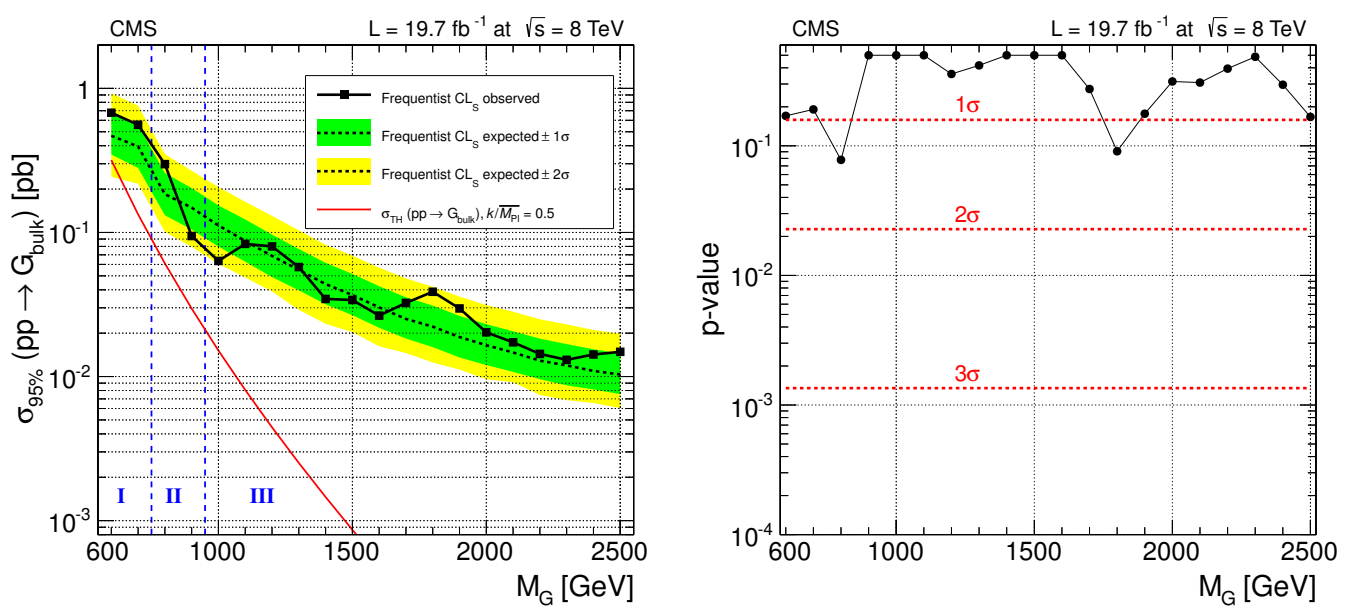

Figure 10. Left: observed (solid) and expected (dashed) 95\% CL upper limit on the graviton production cross section obtained with this analysis and the analysis of the all-hadronic channel [32]. The cross section for the production of a bulk graviton with $k / \bar{M}_{\mathrm{Pl}}=0.5$ is shown as a red solid curve. In region $\mathrm{I}$, only the $\ell \ell+\mathrm{V}$-jet channel contributes. In region $\mathrm{II}$, both $\ell \ell+\mathrm{V}$-jet and $\ell \nu+\mathrm{V}$-jet channels contribute. In region III, both the semi-leptonic and all-hadronic channels contribute. Right: observed p-value as a function of the nominal signal mass. Conversions of the p-value to the number of standard deviations of a two-sided Gaussian distribution are drawn as dashed horizontal red lines.

would be expected to be detected in CMS with the collected integrated luminosity and to compare it with the upper limit on the number of events.

To avoid the dependence on the assumptions in the construction of the separate categories, we perform a simplified analysis, reducing the event classification to one single category. We do this by adding the muon and electron channels and dropping the lowpurity category (whose sensitivity is much smaller than the high-purity category). The loss in performance is very small over a large range of masses. The effect of dropping the LP category is visible only at very high masses, where the upper limit on the cross section becomes $15 \%$ less stringent.

A generic model cannot restrict itself to narrow signal widths, hence we provide limits as a function of both mass $\left(\mathrm{M}_{\mathrm{X}}\right)$ and natural width $\left(\Gamma_{\mathrm{X}}\right)$ of the new resonance. The generated line shape is parametrized with a Breit-Wigner function (BW) and its width is defined as the $\Gamma$ parameter of the BW. The BW line shape is convoluted with the doublesided CB introduced in section 6.2 for describing the detector resolution. While different values of $\Gamma_{\mathrm{X}}$ are scanned, the parameters of the double-CB function are kept fixed to the values determined under the narrow-width approximation. It was checked that the parametrization of the detector effects factorizes from the natural width of the resonance and is stable as $\Gamma_{\mathrm{X}}$ increases. The width scan is done at regular steps of the relative width, $\Gamma_{\mathrm{X}} / \mathrm{M}_{\mathrm{X}}$. The range of values considered spans from the zero width approximation (as in the nominal analysis), up to $\Gamma_{\mathrm{X}} / \mathrm{M}_{\mathrm{X}}=0.40$, in regular steps of 0.05 .

We provide the efficiency as a function of the vector boson kinematic variables, as the efficiency can depend significantly on the production and decay kinematic quantities of the 
new resonance. The efficiencies are extracted from the bulk graviton samples generated for the baseline analysis. The efficiencies are calculated by first preselecting simulated signal events according to the acceptance requirements of the analysis. Thus the usage of the tables is valid only within this kinematic region, summarized in tables 4 and 5 of appendix $\mathrm{A}$ for the $\ell \nu+\mathrm{V}$-jet and $\ell \ell+\mathrm{V}$-jet analyses, respectively. For preselected events, the reconstructed $\mathrm{V}$ candidates are then independently checked to pass the full analysis selection. The efficiency tables are presented as a function of the $p_{\mathrm{T}}$ and $\eta$ of the $\mathrm{V}$ boson from the resonance decay prior to any simulation of detector effects. Bins with fewer than 25 events generated therein are excluded from the final tables. This choice controls the statistical uncertainty of the parametrization and has a very limited impact on the precision of the parametrized efficiencies because they are located in extreme regions of phase space. All the reweighting and rescaling effects (including lepton identification and trigger efficiencies, and V-tagging scale factors) are included in the efficiencies.

The efficiencies of the second-lepton and b-jet vetoes in the $\ell \nu+\mathrm{V}$-jet analysis are found to be independent of the diboson event kinematic in signal events. We use a constant efficiency of $91.5 \%$ for the b-jet veto and $98.3 \%$ for the second-lepton veto, resulting in a total efficiency for the two combined vetoes of $\varepsilon_{\text {vetoes }}=90 \%$.

It was checked that the dependence of the total signal efficiency and acceptance on the width of the generated sample is very mild. We include this effect in the systematic uncertainties of the procedure as discussed later. The resulting efficiencies are presented in figures 11 and 12 for $\mathrm{W}$ and $\mathrm{Z}$ bosons with longitudinal polarization, respectively. The contribution from $\mathrm{Z} \rightarrow \tau \tau$ decays with $\tau \rightarrow \ell \nu \nu$ is not reported since it is suppressed by the dilepton-mass requirement of the analysis described in section 4.5. The same values are presented in tabulated form in appendix A.

Special care must be given to cases where the boson is transversely polarized $\left(\mathrm{V}_{\mathrm{T}}\right)$. The calculated efficiencies are based on longitudinally polarized bosons, as in the case of the reference bulk graviton model. The efficiency of the V-tagging selections depend significantly on the degree of polarization of the vector boson [31]. This effect is investigated with samples of RS1 gravitons produced with the MADGRAPH generator. The V bosons originating from the decays of RS1 gravitons are transversely polarized in about $90 \%$ of the cases. In the cases of bosons decaying leptonically, the tables provided are still valid because of the generator-level selection on the individual leptons, which guarantees that polarization effects for the leptonic boson are included in the acceptance. As shown in ref. [31], the efficiency of the jet substructure selection is found to be lower for transversely polarized $\mathrm{V}$ bosons. Studies of simulated RS1 graviton samples show that the loss of efficiency is largely independent of the $\mathrm{V}$ kinematic variables, so that the effect of the transverse polarization can be adequately modeled by a constant scale factor of 0.85 , independent of the $\eta$ and $p_{\mathrm{T}}$ of the $\mathrm{V} \rightarrow \mathrm{q} \overline{\mathrm{q}}$.

To validate the procedure, the resulting parametrized efficiencies (including the eventveto efficiencies) are used to predict the total efficiency for reconstructing bulk and RS1 gravitons, and the estimation is compared to the exact number obtained from performing the baseline analysis directly on the simulated samples. In all cases, the agreement between the nominal and parametrized efficiencies is within $10 \%$ of their value. Various approxi- 

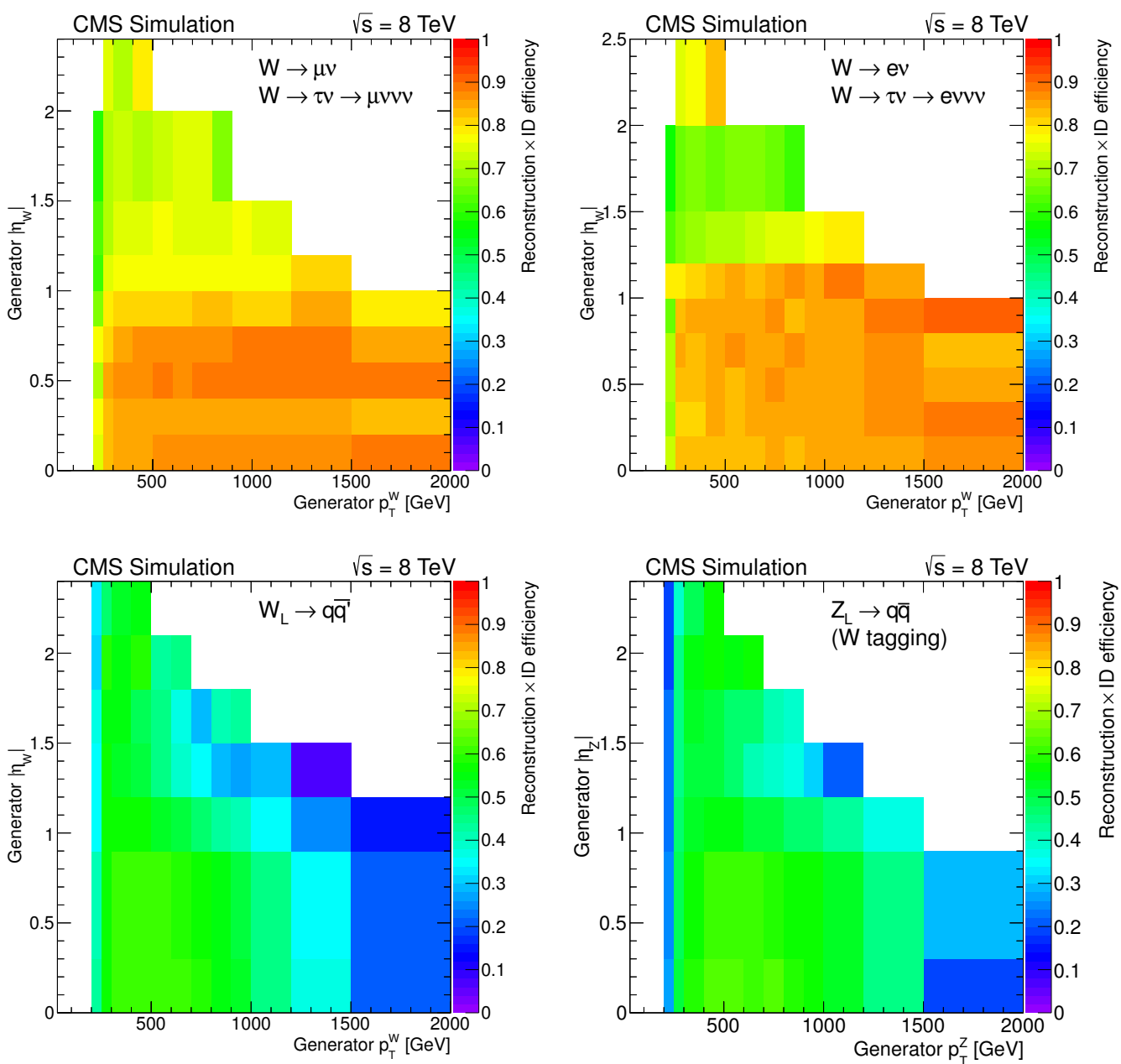

Figure 11. Reconstruction and identification efficiencies for the $\mathrm{W} \rightarrow \mu \nu$ and $\mathrm{W} \rightarrow \tau \nu \rightarrow \mu \nu \nu \nu$ (top left), $\mathrm{W} \rightarrow \mathrm{e} \nu$ and $\mathrm{W} \rightarrow \tau \nu \rightarrow \mathrm{e} \nu \nu \nu$ (top right), $\mathrm{W}_{\mathrm{L}} \rightarrow \mathrm{q} \overline{\mathrm{q}}^{\prime}$ (bottom left), and $\mathrm{Z}_{\mathrm{L}} \rightarrow \mathrm{q} \overline{\mathrm{q}}$ (bottom right) decays as function of generated $p_{\mathrm{T}}^{\mathrm{V}}$ and $\eta_{\mathrm{V}}$ using the $\mathrm{W}$-tagging requirements for the hadronic $\mathrm{V}$ decays.

mations and uncertainties contribute to the final additional systematic uncertainty in the efficiency; the main ones are unaccounted correlations between the physics objects, statistical uncertainties due to limited size of the simulated sample, and residual dependencies on the natural width. We assign an additional systematic uncertainty of $15 \%$ on the total signal efficiency when calculating the model-independent limits. This additional systematic uncertainty conservatively addresses the remaining imperfections in the parametrization of the efficiencies.

Figure 13 and tables 6 and 7 of appendix A show the observed limits on the number of events extracted from the simplified analysis, for the $\ell \nu+\mathrm{V}$-jet and $\ell \ell+\mathrm{V}$-jet analyses independently. The two analyses are not combined in order to avoid assumptions on the branching fractions of a hypothetic resonance decaying to both $\mathrm{WW}$ and $\mathrm{ZZ}$ channels. The limits are calculated using an asymptotic approximation of the $\mathrm{CL}_{S}$ method [86]. Under the narrow-width approximation, it is explicitly checked that the central values for 

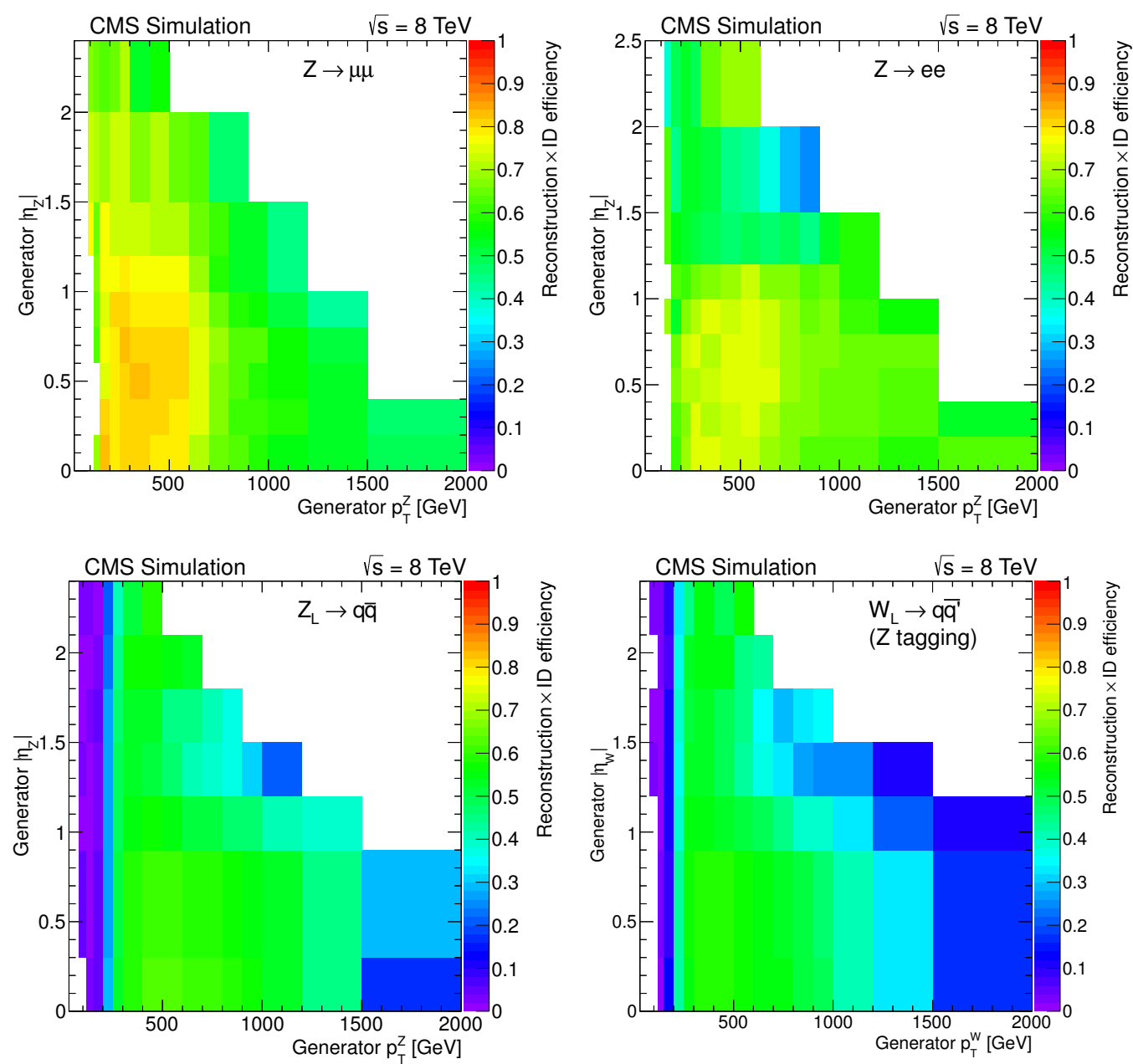

Figure 12. Reconstruction and identification efficiencies for the $\mathrm{Z} \rightarrow \mu \mu$ (top left), $\mathrm{Z} \rightarrow$ ee (top right), $\mathrm{Z}_{\mathrm{L}} \rightarrow \mathrm{q} \overline{\mathrm{q}}$ (bottom left), and $\mathrm{W}_{\mathrm{L}} \rightarrow \mathrm{q} \overline{\mathrm{q}}^{\prime}$ (bottom right) decays as a function of generated $p_{\mathrm{T}}^{\mathrm{V}}$ and $\eta_{\mathrm{V}}$ using the Z-tagging requirements for the hadronic $\mathrm{V}$ decays.

the expected and observed limits returned by the full hybrid frequentist method and the asymptotic approximation match extremely closely over all the range of the search. All the systematic uncertainties considered in the baseline analysis are included in the calculation of these limits, together with the additional 15\% uncertainty related to the approximations used for parametrizing the efficiencies. The main features of the observed limits presented in section 8.1 are still visible. With increasing width, statistical fluctuations in the limit tend to be smoothed out and the overall performance degrades. For relative widths greater than 0.25 , the deterioration of the limit is very mild, because the sensitivity coming from the knowledge of the signal shape is diluted by the very broad signal shape.

Although optimized for WW and ZZ resonances, the analysis is also sensitive to charged resonances decaying to WZ, because of the loose requirement on the V-jet mass. The efficiencies to identify a longitudinally polarized Z $(\mathrm{W})$ boson using $\mathrm{W}$-jet (Z-jet) requirements are computed from the bulk graviton samples using the same procedure described above, 

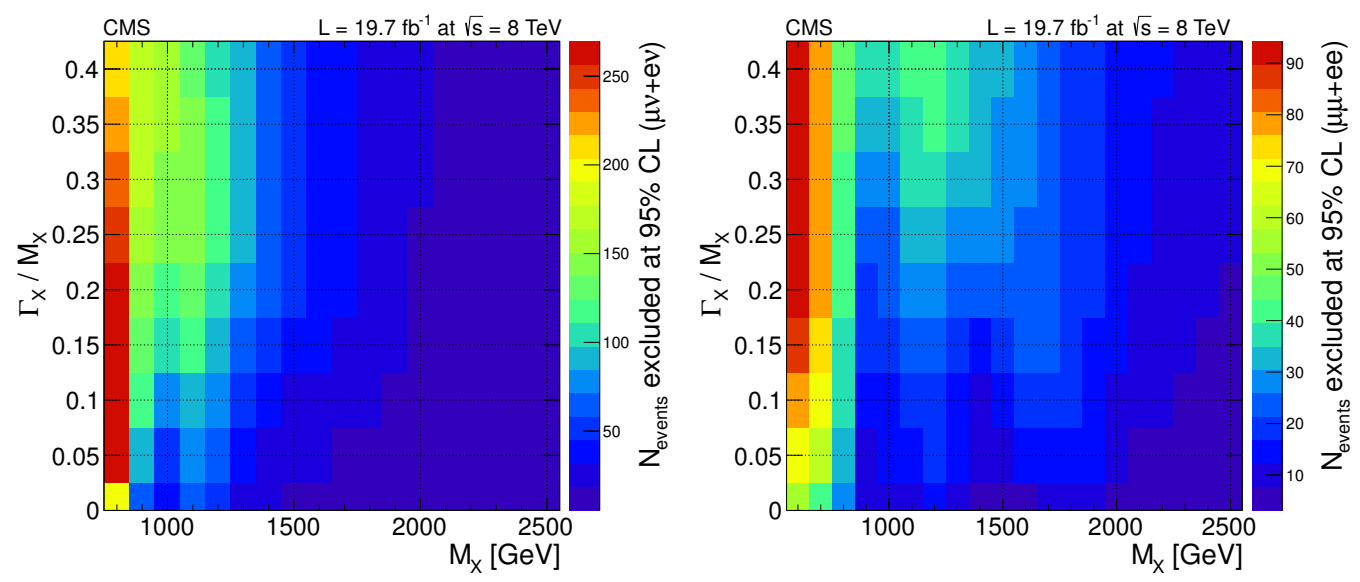

Figure 13. Observed exclusion limits at $95 \% \mathrm{CL}$ on the number of events for a $\mathrm{WV} \rightarrow \ell \nu+V-$ jet (left) and a $\mathrm{ZV} \rightarrow \ell \ell+V$ - jet (right) resonance, as a function of its mass and normalized width.

and they are reported in figure 11 (figure 12). The same values are presented in tabulated form in appendix A. Also in this case, the effect of the transverse polarization of the bosons is modeled by multiplying the aforementioned efficiencies by the constant scale factor 0.85 . In addition, in the $\ell \nu+V$-jet channel, the combined efficiency of the second-lepton veto and b-jet veto becomes $\varepsilon_{\text {vetoes }}=81 \%$, because of the presence of $\mathrm{Z} \rightarrow \mathrm{b} \overline{\mathrm{b}}$ decays, which can be rejected by the requirement of the analysis that no jet is tagged as coming from a b quark.

\section{Summary}

We have presented a search for new resonances decaying to WW, ZZ, or WZ in which one of the bosons decays leptonically and the other hadronically. The final states considered are either $\ell \nu \mathrm{q} \overline{\mathrm{q}}^{(\prime)}$ or $\ell \ell \mathrm{q} \overline{\mathrm{q}}^{(\prime)}$ with $\ell=\mu$ or e. The results include the case in which $\mathrm{W} \rightarrow$ $\tau \nu$ or $\mathrm{Z} \rightarrow \tau \tau$ where the tau decay is $\tau \rightarrow \ell \nu \nu$. The events are reconstructed as a leptonic $\mathrm{W}$ or $\mathrm{Z}$ candidate recoiling against a jet with mass compatible with the $\mathrm{W}$ or $\mathrm{Z}$ mass, respectively. Additional information from jet substructure is used to reduce the background from multijet processes. No evidence for a signal is found, and the result is interpreted as an upper limit on the production cross section as a function of the resonance mass in the context of the bulk graviton model. The final upper limits are based on the statistical combination of the two semi-leptonic channels considered here with those of a complementary search in the fully-hadronic final state. Upper limits at 95\% CL are set on the bulk graviton production cross section in the range from 700 to $10 \mathrm{fb}$ for resonance masses between 600 and $2500 \mathrm{GeV}$, respectively. These limits are the most stringent to date in these final states. The two analyses in the semi-leptonic channels are repeated in a simplified scenario, providing model-independent limits on the number of events. The tabulated efficiency of reconstructing the vector bosons within the kinematic acceptance of the analysis allows the reinterpretation of the exclusion limits in a generic phenomenological model, including WZ resonances, greatly extending the versatility of these results. 


\section{Acknowledgments}

We congratulate our colleagues in the CERN accelerator departments for the excellent performance of the LHC and thank the technical and administrative staffs at CERN and at other CMS institutes for their contributions to the success of the CMS effort. In addition, we gratefully acknowledge the computing centres and personnel of the Worldwide LHC Computing Grid for delivering so effectively the computing infrastructure essential to our analyses. Finally, we acknowledge the enduring support for the construction and operation of the LHC and the CMS detector provided by the following funding agencies: the Austrian Federal Ministry of Science and Research and the Austrian Science Fund; the Belgian Fonds de la Recherche Scientifique, and Fonds voor Wetenschappelijk Onderzoek; the Brazilian Funding Agencies (CNPq, CAPES, FAPERJ, and FAPESP); the Bulgarian Ministry of Education and Science; CERN; the Chinese Academy of Sciences, Ministry of Science and Technology, and National Natural Science Foundation of China; the Colombian Funding Agency (COLCIENCIAS); the Croatian Ministry of Science, Education and Sport; the Research Promotion Foundation, Cyprus; the Ministry of Education and Research, Recurrent financing contract SF0690030s09 and European Regional Development Fund, Estonia; the Academy of Finland, Finnish Ministry of Education and Culture, and Helsinki Institute of Physics; the Institut National de Physique Nucléaire et de Physique des Particules / CNRS, and Commissariat à l'Énergie Atomique et aux Énergies Alternatives / CEA, France; the Bundesministerium für Bildung und Forschung, Deutsche Forschungsgemeinschaft, and Helmholtz-Gemeinschaft Deutscher Forschungszentren, Germany; the General Secretariat for Research and Technology, Greece; the National Scientific Research Foundation, and National Office for Research and Technology, Hungary; the Department of Atomic Energy and the Department of Science and Technology, India; the Institute for Studies in Theoretical Physics and Mathematics, Iran; the Science Foundation, Ireland; the Istituto Nazionale di Fisica Nucleare, Italy; the Korean Ministry of Education, Science and Technology and the World Class University program of NRF, Republic of Korea; the Lithuanian Academy of Sciences; the Mexican Funding Agencies (CINVESTAV, CONACYT, SEP, and UASLP-FAI); the Ministry of Business, Innovation and Employment, New Zealand; the Pakistan Atomic Energy Commission; the Ministry of Science and Higher Education and the National Science Centre, Poland; the Fundação para a Ciência e a Tecnologia, Portugal; JINR, Dubna; the Ministry of Education and Science of the Russian Federation, the Federal Agency of Atomic Energy of the Russian Federation, Russian Academy of Sciences, and the Russian Foundation for Basic Research; the Ministry of Education, Science and Technological Development of Serbia; the Secretaría de Estado de Investigación, Desarrollo e Innovación and Programa Consolider-Ingenio 2010, Spain; the Swiss Funding Agencies (ETH Board, ETH Zurich, PSI, SNF, UniZH, Canton Zurich, and SER); the National Science Council, Taipei; the Thailand Center of Excellence in Physics, the Institute for the Promotion of Teaching Science and Technology of Thailand, Special Task Force for Activating Research and the National Science and Technology Development Agency of Thailand; the Scientific and Technical Research Council of Turkey, and Turkish Atomic Energy Authority; the Science and Technology Facilities Council, U.K.; the US Department of Energy, and the US National Science Foundation. 
Individuals have received support from the Marie-Curie programme and the European Research Council and EPLANET (European Union); the Leventis Foundation; the A. P. Sloan Foundation; the Alexander von Humboldt Foundation; the Belgian Federal Science Policy Office; the Fonds pour la Formation à la Recherche dans l'Industrie et dans l'Agriculture (FRIA-Belgium); the Agentschap voor Innovatie door Wetenschap en Technologie (IWT-Belgium); the Ministry of Education, Youth and Sports (MEYS) of Czech Republic; the Council of Science and Industrial Research, India; the Compagnia di San Paolo (Torino); the HOMING PLUS programme of Foundation for Polish Science, cofinanced by EU, Regional Development Fund; and the Thalis and Aristeia programmes cofinanced by EU-ESF and the Greek NSRF.

\section{A Detailed instructions and additional material for generic interpreta- tion of the results}

This section presents a technical description of the procedure for calculating the signal yield expected to be observed in the CMS detector in a scenario with a new resonance, $\mathrm{X}$, decaying to two vector bosons (WW, ZZ, WZ) in the semi-leptonic final states $\ell \nu+V$-jet and $\ell \ell+\mathrm{V}$-jet. Tables 8 to 10 provide the efficiencies for the reconstruction and identification of each of the two $\mathrm{W}$ vector bosons coming from the $\mathrm{X} \rightarrow \mathrm{WW}$ decay. Tables 11 to 13 provide the efficiencies for the reconstruction and identification of each of the two $\mathrm{Z}$ bosons coming from the $\mathrm{X} \rightarrow \mathrm{ZZ}$ decay. In case of hadronic $\mathrm{V}$ decays from $\mathrm{X} \rightarrow \mathrm{WZ}$ resonances the following tables should be used: table 14 provides the efficiencies for the reconstruction and identification of $\mathrm{Z}_{\mathrm{L}} \rightarrow \mathrm{q} \overline{\mathrm{q}}$ using $\mathrm{W}$-tagging requirements; table 15 provides the efficiencies for the reconstruction and identification of $\mathrm{W}_{\mathrm{L}} \rightarrow \mathrm{q} \overline{\mathrm{q}}^{\prime}$ using Z-tagging requirements. The efficiencies are calculated using the reference bulk graviton samples, listed in section 3 .

These efficiencies can be applied to a generic model with the following procedure:

1. Generate a sample of events for a given mass and width of the $\mathrm{X}$ resonance; the simulated process must include the decay of the $\mathrm{X}$ resonance to leptons and quarks (including $\mathrm{W} \rightarrow \tau \nu \rightarrow \ell \nu \nu \nu$ decays).

2. Filter the events according to the criteria listed in table 4 (if the $\mathrm{X}$ resonance decays to $\mathrm{WW}$ ) and table 5 (if the $\mathrm{X}$ resonance decays to $\mathrm{Z} \mathrm{Z}$ ). If the resonance decays to $\mathrm{WZ} \rightarrow \ell \nu \mathrm{q} \overline{\mathrm{q}}$, the criteria for hadronic $\mathrm{W}$ in table 4 should be applied on the generated hadronic $\mathrm{Z}$. If the resonance decays to $\mathrm{ZW} \rightarrow \ell \ell \mathrm{q} \overline{\mathrm{q}}^{\prime}$, the criteria for hadronic $\mathrm{Z}$ in table 5 should be applied on the generated hadronic W.

3. For each of the remaining events, calculate the efficiency for reconstructing the $\mathrm{W} \rightarrow$ $\mu \nu / \mathrm{W} \rightarrow \tau \nu \rightarrow \mu \nu \nu \nu, \mathrm{W} \rightarrow a \nu / \mathrm{W} \rightarrow \tau \nu \rightarrow \mathrm{e} \nu \nu \nu, \mathrm{Z} \rightarrow \mu \mu$, and $\mathrm{Z} \rightarrow$ ee using the tables $8,9,11$, and 12 , respectively. The tables provide the efficiency parametrized as a function of $p_{\mathrm{T}}$ and $\eta$ of the $\mathrm{W}$ or $\mathrm{Z}$ boson.

4. In a similar way, for WW and ZZ resonances, calculate the efficiency of the hadronic $\mathrm{W}$ or $\mathrm{Z}$ using the values in table 10 or 13 , respectively. If the resonance decays to 


\begin{tabular}{|c|c|}
\hline Object & Requirement \\
\hline \multirow[t]{2}{*}{ Muons } & $|\eta|<2.1$ \\
\hline & $p_{\mathrm{T}}>50 \mathrm{GeV}$ \\
\hline \multirow[t]{2}{*}{ Electrons } & $|\eta|<2.5$ \\
\hline & $p_{\mathrm{T}}>90 \mathrm{GeV}$ \\
\hline$\sum \vec{p}_{\mathrm{T}, \nu}$ (Muon ch.) & $p_{\mathrm{T}}>40 \mathrm{GeV}$ \\
\hline$\sum \vec{p}_{\mathrm{T}, \nu}$ (Electron ch.) & $p_{\mathrm{T}}>80 \mathrm{GeV}$ \\
\hline $\mathrm{W} \rightarrow \ell \nu$ or $W \rightarrow \tau \nu \rightarrow \ell \nu \nu \nu$ & $p_{\mathrm{T}}^{\mathrm{W}}>200 \mathrm{GeV}$ \\
\hline \multirow[t]{3}{*}{$\mathrm{W} \rightarrow \mathrm{q} \overline{\mathrm{q}}^{\prime}$} & $\left|\eta_{\mathrm{W}}\right|<2.4$ \\
\hline & $p_{\mathrm{T}}^{\mathrm{W}}>200 \mathrm{GeV}$ \\
\hline & $65<m_{\mathrm{q}_{\overline{\mathrm{q}}}^{\prime}}<105 \mathrm{GeV}$ \\
\hline \multirow[t]{4}{*}{ WW system } & $700<m_{\mathrm{WW}}<3000 \mathrm{GeV}$ \\
\hline & $\Delta R\left(\mathrm{~W}_{\mathrm{q}^{\prime}}, \ell\right)>\pi / 2$ \\
\hline & $\Delta \phi\left(\mathrm{W}_{\mathrm{q} \overline{\mathrm{q}}^{\prime}}, \sum \vec{p}_{\mathrm{T}, \nu}\right)>2$ \\
\hline & $\Delta \phi\left(\mathrm{W}_{\mathrm{q}_{\overline{\mathrm{q}}}^{\prime}}, \mathrm{W}_{\ell \nu}\right)>2$ \\
\hline
\end{tabular}

Table 4. Generator level requirements for the WW analysis, to be used for the computation of the efficiency parametrization. The vector sum of the transverse neutrino momenta $\sum \vec{p}_{\mathrm{T}, \nu}$ is taken over all the neutrinos in the final state, coming either from $\mathrm{W} \rightarrow \ell \nu$ or $W \rightarrow \tau \nu \rightarrow \ell \nu \nu \nu$ decays with $\ell=\mu$ or e.

$\mathrm{WZ} \rightarrow \ell \nu \mathrm{q} \overline{\mathrm{q}}$, calculate the efficiency of the hadronic $\mathrm{Z}$ using table 14 . If the resonance decays to $\mathrm{ZW} \rightarrow \ell \ell \mathrm{q} \overline{\mathrm{q}}^{\prime}$, calculate the efficiency of the hadronic W using table 15 .

5. Weight each passing event with the product of the two efficiencies found at steps 3 and 4 . In case of a $\mathrm{X}$ resonance decaying to $\mathrm{WW}(\ell \nu+\mathrm{V}$-jet channel), also multiply by the combined efficiency of the second-lepton and b-jet vetoes, equal to $\varepsilon_{\text {vetoes }}=90 \%$. In case of a $\mathrm{X}$ resonance decaying to $\mathrm{WZ} \rightarrow \ell \nu \mathrm{q} \overline{\mathrm{q}}$, the combined efficiency of the two vetoes is equal to $\varepsilon_{\text {vetoes }}=81 \%$.

6. The resulting sum of weights divided by the total number of events provides an approximation to the total efficiency for the given model.

The final number of events can be directly compared to the observed limits in figure 13 and tables 6 and 7, in order to assess the exclusion power of the experiment with respect to the model considered.

The numbers provided refer to longitudinally polarized bosons. For transversely polarized bosons that decay leptonically, the same numbers are valid, as long as they are applied after the kinematic acceptance requirements. If the boson decays to quarks and has a transverse polarization, the efficiency must be scaled down by a factor of 0.85 . 


\begin{tabular}{|lr|}
\hline Object & Requirement \\
\hline Muons & $|\eta|<2.4$ \\
Highest- $p_{\mathrm{T}}$ muon & $p_{\mathrm{T}}>20 \mathrm{GeV}$ \\
& $p_{\mathrm{T}}>40 \mathrm{GeV}$ \\
\hline Electrons & $|\eta|<2.5$ \\
& $p_{\mathrm{T}}>40 \mathrm{GeV}$ \\
\hline $\mathrm{Z} \rightarrow \ell \ell$ & $p_{\mathrm{T}}^{\mathrm{Z}}>80 \mathrm{GeV}$ \\
& $70<m_{\ell \ell}<110 \mathrm{GeV}$ \\
\hline $\mathrm{Z} \rightarrow \mathrm{q} \overline{\mathrm{q}}$ & $\left|\eta_{\mathrm{Z}}\right|<2.4$ \\
& $p_{\mathrm{T}}^{\mathrm{Z}}>80 \mathrm{GeV}$ \\
& $70<m_{\mathrm{q} \overline{\mathrm{q}}}<110 \mathrm{GeV}$ \\
\hline $\mathrm{ZZ}$ system & $500<m_{\mathrm{ZZ}}<2800 \mathrm{GeV}$ \\
\hline
\end{tabular}

Table 5. Generator level requirements for the $\mathrm{Z} \mathrm{Z}$ analysis, to be used for the computation of the efficiency parametrization, with $\ell=\mu$ or e.

\begin{tabular}{|lccccccccc|}
\hline$M_{\mathrm{X}}[\mathrm{GeV}]$ & \multicolumn{8}{c|}{$\Gamma_{\mathrm{X}} / M_{\mathrm{X}}$} \\
& 0.00 & 0.05 & 0.10 & 0.15 & 0.20 & 0.25 & 0.30 & 0.35 & 0.40 \\
\hline 800 & 200 & 260 & 270 & 270 & 260 & 250 & 240 & 230 & 210 \\
900 & 70 & 93 & 113 & 132 & 150 & 160 & 170 & 170 & 170 \\
1000 & 42 & 58 & 77 & 99 & 120 & 139 & 150 & 150 & 154 \\
1100 & 62 & 78 & 95 & 110 & 130 & 139 & 140 & 140 & 137 \\
1200 & 51 & 68 & 82 & 97 & 110 & 120 & 120 & 120 & 110 \\
1300 & 30 & 42 & 54 & 69 & 82 & 89 & 91 & 89 & 85 \\
1400 & 23 & 30 & 39 & 50 & 61 & 67 & 69 & 68 & 66 \\
1500 & 18 & 24 & 32 & 41 & 48 & 52 & 53 & 53 & 51 \\
1600 & 14 & 19 & 26 & 34 & 39 & 42 & 42 & 42 & 41 \\
1700 & 13 & 18 & 24 & 29 & 32 & 33 & 34 & 34 & 33 \\
1800 & 12 & 16 & 21 & 24 & 26 & 27 & 28 & 28 & 27 \\
1900 & 8.7 & 13 & 16 & 19 & 21 & 22 & 22 & 22 & 22 \\
2000 & 8.3 & 11 & 14 & 16 & 17 & 18 & 19 & 19 & 19 \\
2100 & 7.5 & 9.5 & 12 & 13 & 14 & 15 & 16 & 16 & 16 \\
2200 & 5.3 & 7.8 & 9.8 & 11 & 12 & 13 & 13 & 14 & 14 \\
2300 & 5.4 & 7.1 & 8.6 & 9.7 & 11 & 11 & 12 & 12 & 13 \\
2400 & 5.6 & 6.8 & 7.8 & 8.7 & 9.4 & 9.9 & 10 & 11 & 11 \\
2500 & 5.3 & 6.5 & 7.3 & 8.0 & 8.6 & 9.1 & 9.6 & 10 & 10 \\
\hline
\end{tabular}

Table 6. Simplified limits on the number of visible events from generic WV resonances in the $\ell \nu+\mathrm{V}$-jet channel as a function of resonance mass, $M_{\mathrm{X}}$, and normalized width, $\Gamma_{\mathrm{X}} / M_{\mathrm{X}}$. Shown are limits on the visible number of events at $95 \% \mathrm{CL}$ using the asymptotic $\mathrm{CL}_{S}$ approach. Results with $\Gamma_{\mathrm{X}} / M_{\mathrm{X}}=0$ are obtained using the resolution function only. 


\begin{tabular}{|lccccccccc|}
\hline$M_{\mathrm{X}}[\mathrm{GeV}]$ & \multicolumn{10}{c|}{$\Gamma_{\mathrm{X}} / M_{\mathrm{X}}$} \\
& 0.00 & 0.05 & 0.10 & 0.15 & 0.20 & 0.25 & 0.30 & 0.35 & 0.40 \\
\hline 600 & 53 & 68 & 78 & 85 & 90 & 93 & 94 & 94 & 93 \\
700 & 43 & 58 & 69 & 76 & 79 & 80 & 79 & 78 & 77 \\
800 & 27 & 33 & 37 & 39 & 41 & 42 & 44 & 46 & 48 \\
900 & 8.4 & 11 & 14 & 17 & 21 & 24 & 28 & 32 & 36 \\
1000 & 11 & 14 & 16 & 19 & 22 & 25 & 29 & 33 & 37 \\
1100 & 12 & 16 & 20 & 24 & 28 & 32 & 36 & 39 & 42 \\
1200 & 14 & 18 & 21 & 25 & 29 & 33 & 37 & 41 & 43 \\
1300 & 11 & 13 & 16 & 20 & 25 & 30 & 35 & 37 & 38 \\
1400 & 5.2 & 7.9 & 11 & 16 & 22 & 28 & 31 & 33 & 33 \\
1500 & 7.0 & 9.7 & 14 & 20 & 25 & 28 & 30 & 30 & 31 \\
1600 & 7.5 & 12 & 18 & 22 & 25 & 26 & 26 & 27 & 27 \\
1700 & 9.6 & 15 & 20 & 22 & 23 & 23 & 24 & 24 & 24 \\
1800 & 10 & 15 & 18 & 19 & 20 & 20 & 21 & 21 & 21 \\
1900 & 9.5 & 13 & 15 & 16 & 17 & 18 & 18 & 18 & 18 \\
2000 & 6.3 & 9.5 & 12 & 14 & 14 & 15 & 15 & 16 & 16 \\
2100 & 3.3 & 5.8 & 9.3 & 11 & 12 & 13 & 13 & 14 & 14 \\
2200 & 3.1 & 5.4 & 7.9 & 9.2 & 10 & 11 & 12 & 12 & 13 \\
2300 & 4.3 & 6.4 & 7.4 & 8.2 & 9.0 & 9.6 & 10 & 11 & 11 \\
2400 & 5.9 & 6.5 & 7.0 & 7.6 & 8.2 & 8.8 & 9.3 & 9.9 & 10 \\
2500 & 5.9 & 6.3 & 6.7 & 7.1 & 7.6 & 8.1 & 8.6 & 9.1 & 9.6 \\
\hline
\end{tabular}

Table 7. Simplified limits on the number of visible events from generic ZV resonances in the $\ell \ell+\mathrm{V}$-jet channel as a function of resonance mass, $M_{\mathrm{X}}$, and normalized width, $\Gamma_{\mathrm{X}} / M_{\mathrm{X}}$. Shown are limits on the visible number of events at $95 \% \mathrm{CL}$ using the asymptotic $\mathrm{CL}_{S}$ approach. Results with $\Gamma_{\mathrm{X}} / M_{\mathrm{X}}=0$ are obtained using the resolution function only.

\begin{tabular}{|lccccccccccc|}
\hline \multirow{2}{*}{$p_{\mathrm{T}}^{\mathrm{W}}$ range $[\mathrm{GeV}]$} & \multicolumn{9}{c}{$\left|\eta_{\mathrm{W}}\right|$ range } \\
& $0.0-0.2$ & $0.2-0.4$ & $0.4-0.6$ & $0.6-0.8$ & $0.8-1.0$ & $1.0-1.2$ & $1.2-1.5$ & $1.5-2.0$ & $2.0-2.4$ & $2.4-3.0$ \\
\hline $200-250$ & 0.75 & 0.78 & 0.70 & 0.76 & 0.66 & 0.60 & 0.63 & 0.59 & - & - \\
$250-300$ & 0.84 & 0.83 & 0.85 & 0.82 & 0.79 & 0.76 & 0.71 & 0.71 & 0.75 & - \\
$300-400$ & 0.85 & 0.86 & 0.86 & 0.86 & 0.81 & 0.77 & 0.74 & 0.74 & 0.71 & - \\
$400-500$ & 0.86 & 0.86 & 0.87 & 0.86 & 0.81 & 0.77 & 0.75 & 0.71 & 0.79 & - \\
$500-600$ & 0.86 & 0.85 & 0.88 & 0.86 & 0.82 & 0.77 & 0.76 & 0.73 & - & - \\
$600-700$ & 0.87 & 0.85 & 0.88 & 0.87 & 0.82 & 0.77 & 0.76 & 0.74 & - & - \\
$700-800$ & 0.86 & 0.85 & 0.88 & 0.86 & 0.82 & 0.77 & 0.75 & 0.74 & - & - \\
$800-900$ & 0.86 & 0.84 & 0.88 & 0.88 & 0.82 & 0.78 & 0.75 & 0.67 & - & - \\
$900-1000$ & 0.87 & 0.85 & 0.89 & 0.88 & 0.81 & 0.77 & 0.76 & - & - & - \\
$1000-1200$ & 0.87 & 0.85 & 0.90 & 0.88 & 0.82 & 0.77 & 0.76 & - & - & - \\
$1200-1500$ & 0.87 & 0.85 & 0.90 & 0.89 & 0.84 & 0.80 & - & - & - \\
$1500-2000$ & 0.90 & 0.82 & 0.89 & 0.85 & 0.80 & - & - & - & - & - \\
\hline
\end{tabular}

Table 8. Reconstruction and identification efficiency for the $\mathrm{W} \rightarrow \mu \nu$ and $\mathrm{W} \rightarrow \tau \nu \rightarrow \mu \nu \nu \nu$ decays as function of generated $p_{\mathrm{T}}^{\mathrm{W}}$ and $\left|\eta_{\mathrm{W}}\right|$. Uncertainties on the efficiencies are included in the generic limit calculation as discussed in the text. 


\begin{tabular}{|lccccccccccc|}
\hline$p_{\mathrm{T}}^{\mathrm{W}}$ range $[\mathrm{GeV}]$ & \multicolumn{10}{c|}{$\left|\eta_{\mathrm{W}}\right|$ range } \\
& $0.0-0.2$ & $0.2-0.4$ & $0.4-0.6$ & $0.6-0.8$ & $0.8-1.0$ & $1.0-1.2$ & $1.2-1.5$ & $1.5-2.0$ & $2.0-2.5$ & $2.5-3.0$ \\
\hline $200-250$ & 0.73 & 0.68 & 0.70 & 0.71 & 0.65 & 0.80 & 0.62 & 0.57 & - & - \\
$250-300$ & 0.84 & 0.80 & 0.83 & 0.85 & 0.84 & 0.79 & 0.67 & 0.65 & 0.75 & - \\
$300-400$ & 0.84 & 0.82 & 0.83 & 0.83 & 0.85 & 0.81 & 0.69 & 0.68 & 0.76 & - \\
$400-500$ & 0.84 & 0.85 & 0.86 & 0.85 & 0.85 & 0.83 & 0.71 & 0.65 & 0.83 & - \\
$500-600$ & 0.84 & 0.83 & 0.83 & 0.86 & 0.84 & 0.84 & 0.72 & 0.68 & - & - \\
$600-700$ & 0.83 & 0.85 & 0.85 & 0.85 & 0.84 & 0.83 & 0.73 & 0.68 & - & - \\
$700-800$ & 0.85 & 0.85 & 0.87 & 0.85 & 0.86 & 0.84 & 0.75 & 0.65 & - & - \\
$800-900$ & 0.84 & 0.85 & 0.85 & 0.87 & 0.84 & 0.86 & 0.75 & 0.61 & - & - \\
$900-1000$ & 0.84 & 0.85 & 0.86 & 0.85 & 0.84 & 0.85 & 0.76 & - & - \\
$1000-1200$ & 0.84 & 0.86 & 0.86 & 0.86 & 0.85 & 0.89 & 0.78 & - & - \\
$1200-1500$ & 0.86 & 0.86 & 0.88 & 0.86 & 0.89 & 0.84 & - & - & - \\
$1500-2000$ & 0.87 & 0.90 & 0.85 & 0.84 & 0.91 & - & - & - & - \\
\hline
\end{tabular}

Table 9. Reconstruction and identification efficiency for the $\mathrm{W} \rightarrow \mathrm{e} \nu$ and $\mathrm{W} \rightarrow \tau \nu \rightarrow \mathrm{e} \nu \nu \nu$ decays as a function of generated $p_{\mathrm{T}}^{\mathrm{W}}$ and $\left|\eta_{\mathrm{W}}\right|$. Uncertainties on the efficiencies are included in the generic limit calculation as discussed in the text.

\begin{tabular}{|lccccccc|}
\hline$p_{\mathrm{T}}^{\mathrm{W}}$ range $[\mathrm{GeV}]$ & \multicolumn{7}{c|}{$\left|\eta_{\mathrm{W}}\right|$ range } \\
\hline $200-250$ & $0.0-0.3$ & $0.3-0.9$ & $0.9-1.2$ & $1.2-1.5$ & $1.5-1.8$ & $1.8-2.1$ & $2.1-2.4$ \\
$250-300$ & 0.43 & 0.40 & 0.35 & 0.35 & 0.37 & 0.32 & 0.33 \\
$300-400$ & 0.58 & 0.57 & 0.56 & 0.56 & 0.56 & 0.59 & 0.46 \\
$400-500$ & 0.62 & 0.61 & 0.57 & 0.54 & 0.55 & 0.55 & 0.53 \\
$500-600$ & 0.61 & 0.61 & 0.56 & 0.50 & 0.50 & 0.58 & 0.54 \\
$600-700$ & 0.61 & 0.60 & 0.52 & 0.45 & 0.44 & 0.43 & - \\
$700-800$ & 0.60 & 0.59 & 0.52 & 0.39 & 0.38 & 0.45 & - \\
$800-900$ & 0.59 & 0.57 & 0.47 & 0.35 & 0.29 & - & - \\
$900-1000$ & 0.55 & 0.55 & 0.42 & 0.30 & 0.41 & - & - \\
$1000-1200$ & 0.52 & 0.51 & 0.41 & 0.28 & 0.43 & - & - \\
$1200-1500$ & 0.45 & 0.44 & 0.35 & 0.28 & - & - & - \\
$1500-2000$ & 0.36 & 0.35 & 0.24 & 0.07 & - & - & - \\
\hline
\end{tabular}

Table 10. Reconstruction and identification efficiency for the $\mathrm{W}_{\mathrm{L}} \rightarrow \mathrm{q} \overline{\mathrm{q}}^{\prime}$ decay as a function of generated $p_{\mathrm{T}}^{\mathrm{W}}$ and $\left|\eta_{\mathrm{W}}\right|$ using $\mathrm{W}$-tagging requirements. Uncertainties on the efficiencies are included in the generic limit calculation as discussed in the text. 


\begin{tabular}{|lcccccccccc|}
\hline$p_{\text {T }}^{Z}$ range $[\mathrm{GeV}]$ & \multicolumn{10}{c|}{$\left|\eta_{Z}\right|$ range } \\
& $0.0-0.2$ & $0.2-0.4$ & $0.4-0.6$ & $0.6-0.8$ & $0.8-1.0$ & $1.0-1.2$ & $1.2-1.5$ & $1.5-2.0$ & $2.0-2.4$ & $2.4-3.0$ \\
\hline $90-120$ & - & - & - & - & - & - & 0.78 & 0.73 & 0.67 & - \\
$120-150$ & 0.68 & - & - & 0.63 & 0.66 & 0.67 & 0.65 & 0.70 & 0.65 & - \\
$150-200$ & 0.82 & 0.80 & 0.74 & 0.78 & 0.74 & 0.78 & 0.77 & 0.70 & 0.64 & - \\
$200-250$ & 0.79 & 0.79 & 0.78 & 0.79 & 0.80 & 0.77 & 0.74 & 0.73 & 0.64 & - \\
$250-300$ & 0.81 & 0.80 & 0.81 & 0.84 & 0.80 & 0.79 & 0.74 & 0.72 & 0.70 & - \\
$300-400$ & 0.80 & 0.81 & 0.82 & 0.82 & 0.79 & 0.78 & 0.73 & 0.68 & 0.53 & - \\
$400-500$ & 0.79 & 0.81 & 0.82 & 0.81 & 0.79 & 0.76 & 0.72 & 0.71 & 0.58 & - \\
$500-600$ & 0.78 & 0.79 & 0.81 & 0.81 & 0.78 & 0.77 & 0.71 & 0.64 & - & - \\
$600-700$ & 0.72 & 0.75 & 0.76 & 0.74 & 0.72 & 0.69 & 0.65 & 0.62 & - & - \\
$700-800$ & 0.65 & 0.67 & 0.68 & 0.68 & 0.64 & 0.60 & 0.62 & 0.46 & - \\
$800-900$ & 0.62 & 0.62 & 0.63 & 0.63 & 0.58 & 0.53 & 0.54 & 0.47 & - \\
$900-1000$ & 0.58 & 0.62 & 0.59 & 0.62 & 0.56 & 0.54 & 0.52 & - & - \\
$1000-1200$ & 0.55 & 0.58 & 0.58 & 0.57 & 0.51 & 0.45 & 0.46 & - & - \\
$1200-1500$ & 0.54 & 0.54 & 0.53 & 0.51 & 0.43 & - & - & - & - \\
$1500-2000$ & 0.49 & 0.46 & - & - & - & - & - & - & - \\
\hline
\end{tabular}

Table 11. Reconstruction and identification efficiency for the $\mathrm{Z} \rightarrow \mu \mu$ decay as a function of generated $p_{\mathrm{T}}^{\mathrm{Z}}$ and $\left|\eta_{\mathrm{Z}}\right|$. Uncertainties on the efficiencies are included in the generic limit calculation as discussed in the text.

\begin{tabular}{|lcccccccccc|}
\hline$p_{\mathrm{T}}^{\mathrm{Z}}$ range $[\mathrm{GeV}]$ & \multicolumn{10}{c|}{$\left|\eta_{\mathrm{Z}}\right|$ range } \\
& $0.0-0.2$ & $0.2-0.4$ & $0.4-0.6$ & $0.6-0.8$ & $0.8-1.0$ & $1.0-1.2$ & $1.2-1.5$ & $1.5-2.0$ & $2.0-2.5$ & $2.5-3.0$ \\
\hline $120-150$ & - & - & - & - & 0.70 & - & 0.62 & 0.63 & 0.39 & - \\
$150-200$ & 0.61 & 0.64 & 0.66 & 0.65 & 0.53 & 0.62 & 0.54 & 0.46 & 0.48 & - \\
$200-250$ & 0.70 & 0.67 & 0.71 & 0.72 & 0.68 & 0.60 & 0.53 & 0.53 & 0.53 & - \\
$250-300$ & 0.74 & 0.74 & 0.71 & 0.76 & 0.72 & 0.65 & 0.51 & 0.54 & 0.48 & - \\
$300-400$ & 0.74 & 0.72 & 0.73 & 0.71 & 0.74 & 0.67 & 0.49 & 0.51 & 0.65 & - \\
$400-500$ & 0.73 & 0.73 & 0.74 & 0.75 & 0.73 & 0.70 & 0.47 & 0.44 & 0.68 & - \\
$500-600$ & 0.74 & 0.75 & 0.75 & 0.75 & 0.75 & 0.72 & 0.50 & 0.43 & 0.68 & - \\
$600-700$ & 0.69 & 0.71 & 0.74 & 0.73 & 0.73 & 0.68 & 0.47 & 0.37 & - & - \\
$700-800$ & 0.67 & 0.68 & 0.68 & 0.70 & 0.68 & 0.67 & 0.48 & 0.29 & - & - \\
$800-900$ & 0.64 & 0.67 & 0.67 & 0.67 & 0.65 & 0.64 & 0.46 & 0.25 & - & - \\
$900-1000$ & 0.63 & 0.65 & 0.65 & 0.66 & 0.67 & 0.66 & 0.54 & - & - & - \\
$1000-1200$ & 0.63 & 0.64 & 0.65 & 0.65 & 0.60 & 0.58 & 0.58 & - & - \\
$1200-1500$ & 0.60 & 0.63 & 0.63 & 0.66 & 0.58 & - & - & - & - & - \\
$1500-2000$ & 0.63 & 0.54 & - & - & - & - & - & - & - & - \\
\hline
\end{tabular}

Table 12. Reconstruction and identification efficiency for the $\mathrm{Z} \rightarrow$ ee decay as a function of generated $p_{\mathrm{T}}^{\mathrm{Z}}$ and $\left|\eta_{\mathrm{Z}}\right|$. Uncertainties on the efficiencies are included in the generic limit calculation as discussed in the text. 


\begin{tabular}{|lccccccc|}
\hline$p_{\text {T }}^{\mathrm{Z}}$ range $[\mathrm{GeV}]$ & \multicolumn{7}{c|}{$\left|\eta_{\mathrm{Z}}\right|$ range } \\
& $0.0-0.3$ & $0.3-0.9$ & $0.9-1.2$ & $1.2-1.5$ & $1.5-1.8$ & $1.8-2.1$ & $2.1-2.4$ \\
\hline $80-120$ & - & 0.04 & 0.02 & 0.02 & 0.01 & 0.02 & 0.04 \\
$120-150$ & 0.03 & 0.02 & 0.01 & 0.01 & 0.02 & 0.01 & 0.03 \\
$150-200$ & 0.05 & 0.05 & 0.04 & 0.03 & 0.04 & 0.04 & 0.02 \\
$200-250$ & 0.29 & 0.28 & 0.27 & 0.27 & 0.26 & 0.22 & 0.20 \\
$250-300$ & 0.53 & 0.52 & 0.49 & 0.49 & 0.47 & 0.44 & 0.40 \\
$300-400$ & 0.60 & 0.59 & 0.55 & 0.52 & 0.53 & 0.56 & 0.51 \\
$400-500$ & 0.62 & 0.61 & 0.57 & 0.52 & 0.52 & 0.56 & 0.59 \\
$500-600$ & 0.62 & 0.62 & 0.55 & 0.47 & 0.46 & 0.55 & - \\
$600-700$ & 0.61 & 0.60 & 0.51 & 0.41 & 0.45 & 0.54 & - \\
$700-800$ & 0.62 & 0.59 & 0.51 & 0.40 & 0.40 & - & - \\
$800-900$ & 0.59 & 0.57 & 0.48 & 0.37 & 0.38 & - & - \\
$900-1000$ & 0.57 & 0.56 & 0.47 & 0.31 & - & - & - \\
$1000-1200$ & 0.53 & 0.52 & 0.41 & 0.22 & - & - & - \\
$1200-1500$ & 0.45 & 0.45 & 0.38 & - & - & - & - \\
$1500-2000$ & 0.18 & 0.30 & - & - & - & - & - \\
\hline
\end{tabular}

Table 13. Reconstruction and identification efficiency for the $Z_{L} \rightarrow q \bar{q}$ decay as a function of generated $p_{T}^{Z}$ and $\left|\eta_{Z}\right|$ using Z-tagging requirements. Uncertainties on the efficiencies are included in the generic limit calculation as discussed in the text.

\begin{tabular}{|lccccccc|}
\hline$p_{\text {T }}^{\text {Z range }[\mathrm{GeV}]}$ & \multicolumn{7}{c|}{$\left|\eta_{\text {Z }}\right|$ range } \\
& $0.0-0.3$ & $0.3-0.9$ & $0.9-1.2$ & $1.2-1.5$ & $1.5-1.8$ & $1.8-2.1$ & $2.1-2.4$ \\
\hline $200-250$ & 0.27 & 0.25 & 0.23 & 0.23 & 0.24 & 0.20 & 0.19 \\
$250-300$ & 0.51 & 0.50 & 0.46 & 0.45 & 0.46 & 0.45 & 0.39 \\
$300-400$ & 0.59 & 0.58 & 0.53 & 0.51 & 0.52 & 0.55 & 0.50 \\
$400-500$ & 0.62 & 0.61 & 0.55 & 0.51 & 0.51 & 0.57 & 0.58 \\
$500-600$ & 0.63 & 0.62 & 0.55 & 0.47 & 0.48 & 0.56 & - \\
$600-700$ & 0.61 & 0.60 & 0.51 & 0.41 & 0.47 & 0.57 & - \\
$700-800$ & 0.62 & 0.60 & 0.51 & 0.38 & 0.41 & - & - \\
$800-900$ & 0.60 & 0.58 & 0.48 & 0.37 & 0.38 & - & - \\
$900-1000$ & 0.57 & 0.56 & 0.47 & 0.31 & - & - & - \\
$1000-1200$ & 0.54 & 0.53 & 0.42 & 0.21 & - & - & - \\
$1200-1500$ & 0.46 & 0.46 & 0.38 & - & - & - & - \\
$1500-2000$ & 0.19 & 0.29 & - & - & - & - & - \\
\hline
\end{tabular}

Table 14. Reconstruction and identification efficiency for the $Z_{L} \rightarrow q \bar{q}$ decay as a function of generated $p_{T}^{Z}$ and $\left|\eta_{Z}\right|$ using $\mathrm{W}$-tagging requirements. Uncertainties on the efficiencies are included in the generic limit calculation as discussed in the text. 


\begin{tabular}{|lccccccc|}
\hline$p_{\mathrm{T}}^{\mathrm{W}}$ range $[\mathrm{GeV}]$ & \multicolumn{7}{c|}{$\left|\eta_{\mathrm{W}}\right|$ range } \\
& $0.0-0.3$ & $0.3-0.9$ & $0.9-1.2$ & $1.2-1.5$ & $1.5-1.8$ & $1.8-2.1$ & $2.1-2.4$ \\
\hline $80-120$ & - & - & - & 0.02 & 0.01 & - & 0.02 \\
$120-150$ & 0.01 & 0.02 & 0.02 & 0.01 & 0.01 & 0.02 & 0.04 \\
$150-200$ & 0.13 & 0.09 & 0.11 & 0.10 & 0.11 & 0.07 & 0.10 \\
$200-250$ & 0.44 & 0.43 & 0.40 & 0.37 & 0.39 & 0.37 & 0.33 \\
$250-300$ & 0.56 & 0.57 & 0.54 & 0.53 & 0.51 & 0.54 & 0.45 \\
$300-400$ & 0.59 & 0.59 & 0.56 & 0.52 & 0.53 & 0.55 & 0.55 \\
$400-500$ & 0.59 & 0.58 & 0.53 & 0.48 & 0.48 & 0.54 & 0.49 \\
$500-600$ & 0.57 & 0.57 & 0.50 & 0.42 & 0.44 & 0.45 & 0.56 \\
$600-700$ & 0.56 & 0.55 & 0.49 & 0.36 & 0.36 & 0.42 & - \\
$700-800$ & 0.55 & 0.53 & 0.45 & 0.33 & 0.30 & - & - \\
$800-900$ & 0.52 & 0.52 & 0.40 & 0.27 & 0.34 & - & - \\
$900-1000$ & 0.48 & 0.47 & 0.38 & 0.26 & 0.35 & - & - \\
$1000-1200$ & 0.42 & 0.41 & 0.33 & 0.26 & - & - & - \\
$1200-1500$ & 0.32 & 0.33 & 0.22 & 0.10 & - & - & - \\
$1500-2000$ & 0.18 & 0.18 & 0.11 & - & - & - & - \\
\hline
\end{tabular}

Table 15. Reconstruction and identification efficiency for the $\mathrm{W}_{\mathrm{L}} \rightarrow \mathrm{q} \overline{\mathrm{q}}^{\prime}$ decay as a function of generated $p_{\mathrm{T}}^{\mathrm{W}}$ and $\left|\eta_{\mathrm{W}}\right|$ using Z-tagging requirements. Uncertainties on the efficiencies are included in the generic limit calculation as discussed in the text.

Open Access. This article is distributed under the terms of the Creative Commons Attribution License (CC-BY 4.0), which permits any use, distribution and reproduction in any medium, provided the original author(s) and source are credited.

\section{References}

[1] F. Englert and R. Brout, Broken symmetry and the mass of gauge vector mesons, Phys. Rev. Lett. 13 (1964) 321 [INSPIRE].

[2] P.W. Higgs, Broken symmetries, massless particles and gauge fields, Phys. Lett. 12 (1964) 132 [INSPIRE].

[3] P.W. Higgs, Broken symmetries and the masses of gauge bosons, Phys. Rev. Lett. 13 (1964) 508 [INSPIRE].

[4] G.S. Guralnik, C.R. Hagen and T.W.B. Kibble, Global conservation laws and massless particles, Phys. Rev. Lett. 13 (1964) 585 [InSPIRE].

[5] P.W. Higgs, Spontaneous symmetry breakdown without massless bosons, Phys. Rev. 145 (1966) 1156 [INSPIRE].

[6] T.W.B. Kibble, Symmetry breaking in non-abelian gauge theories, Phys. Rev. 155 (1967) 1554 [INSPIRE]. 
[7] CMS collaboration, Observation of a new boson at a mass of 125 GeV with the CMS experiment at the LHC, Phys. Lett. B 716 (2012) 30 [arXiv:1207.7235] [INSPIRE].

[8] CMS collaboration, Observation of a new boson with mass near $125 \mathrm{GeV}$ in pp collisions at $\sqrt{s}=7$ and $8 \mathrm{TeV}$, JHEP 06 (2013) 081 [arXiv:1303.4571] [INSPIRE].

[9] ATLAS collaboration, Observation of a new particle in the search for the standard model Higgs boson with the ATLAS detector at the LHC, Phys. Lett. B 716 (2012) 1 [arXiv: 1207.7214] [INSPIRE].

[10] S. Weinberg, Implications of dynamical symmetry breaking, Phys. Rev. D 13 (1976) 974 [INSPIRE].

[11] S. Weinberg, Implications of dynamical symmetry breaking: an addendum, Phys. Rev. D 19 (1979) 1277 [INSPIRE].

[12] L. Susskind, Dynamics of spontaneous symmetry breaking in the Weinberg-Salam theory, Phys. Rev. D 20 (1979) 2619 [INSPIRE].

[13] D.B. Kaplan and H. Georgi, SU(2) × U(1) breaking by vacuum misalignment, Phys. Lett. B 136 (1984) 183 [INSPIRE].

[14] R. Contino, T. Kramer, M. Son and R. Sundrum, Warped/composite phenomenology simplified, JHEP 05 (2007) 074 [hep-ph/0612180] [INSPIRE].

[15] G.F. Giudice, C. Grojean, A. Pomarol and R. Rattazzi, The strongly-interacting light Higgs, JHEP 06 (2007) 045 [hep-ph/0703164] [INSPIRE].

[16] CMS collaboration, Search for a $W^{\prime}$ or techni- $\rho$ decaying into $W Z$ in pp collisions at $\sqrt{s}=7$ TeV, Phys. Rev. Lett. 109 (2012) 141801 [arXiv:1206.0433] [InSPIRE].

[17] CMS collaboration, Search for a narrow spin-2 resonance decaying to a pair of $Z$ vector bosons in the semileptonic final state, Phys. Lett. B 718 (2013) 1208 [arXiv:1209.3807] [INSPIRE].

[18] CMS collaboration, Search for exotic resonances decaying into $W Z / Z Z$ in pp collisions at $\sqrt{s}=7 \mathrm{TeV}$, JHEP 02 (2013) 036 [arXiv:1211.5779] [INSPIRE].

[19] CMS collaboration, Search for heavy resonances in the $W / Z$-tagged dijet mass spectrum in pp collisions at 7 TeV, Phys. Lett. B 723 (2013) 280 [arXiv:1212.1910] [InSPIRE].

[20] ATLAS collaboration, Search for new particles decaying to $Z Z$ using final states with leptons and jets with the ATLAS detector in $\sqrt{s}=7$ TeV proton-proton collisions, Phys. Lett. B 712 (2012) 331 [arXiv:1203.0718] [INSPIRE].

[21] ATLAS collaboration, Search for resonant $W Z$ production in the $W Z \rightarrow \ell \nu \ell^{\prime} \ell^{\prime}$ channel in $\sqrt{s}=7$ TeV pp collisions with the ATLAS detector, Phys. Rev. D 85 (2012) 112012 [arXiv:1204.1648] [INSPIRE].

[22] ATLAS collaboration, Search for new phenomena in the $W W$ to $\ell \nu \ell^{\prime} \nu^{\prime}$ final state in $p p$ collisions at $\sqrt{s}=7 \mathrm{TeV}$ with the ATLAS detector, Phys. Lett. B 718 (2013) 860 [arXiv: 1208.2880] [INSPIRE].

[23] ATLAS collaboration, Search for resonant diboson production in the $W W / W Z \rightarrow \ell \nu j j$ decay channels with the ATLAS detector at $\sqrt{s}=7$ TeV, Phys. Rev. D 87 (2013) 112006 [arXiv:1305.0125] [INSPIRE].

[24] C. Delaunay, O. Gedalia, S.J. Lee, G. Perez and E. Ponton, Ultra visible warped model from 
flavor triviality and improved naturalness, Phys. Rev. D 83 (2011) 115003

[arXiv: 1007.0243] [INSPIRE].

[25] K. Agashe, A. Delgado, M.J. May and R. Sundrum, RS1, custodial isospin and precision tests, JHEP 08 (2003) 050 [hep-ph/0308036] [INSPIRE].

[26] L. Randall and R. Sundrum, A large mass hierarchy from a small extra dimension, Phys. Rev. Lett. 83 (1999) 3370 [hep-ph/9905221] [INSPIRE].

[27] K. Agashe, H. Davoudiasl, G. Perez and A. Soni, Warped gravitons at the LHC and beyond, Phys. Rev. D 76 (2007) 036006 [hep-ph/0701186] [InSPIRE].

[28] A.L. Fitzpatrick, J. Kaplan, L. Randall and L.-T. Wang, Searching for the Kaluza-Klein graviton in bulk RS models, JHEP 09 (2007) 013 [hep-ph/0701150] [INSPIRE].

[29] O. Antipin, D. Atwood and A. Soni, Search for RS gravitons via $W_{L} W_{L}$ decays, Phys. Lett. B 666 (2008) 155 [arXiv:0711.3175] [InSPIRE].

[30] A. Oliveira, Gravity particles from warped extra dimensions, a review. Part I - KK graviton, arXiv: 1404.0102 [INSPIRE].

[31] CMS collaboration, Identifying Hadronically decaying vector bosons merged into a single jet, CMS-PAS-JME-13-006 (2013).

[32] CMS collaboration, Search for massive resonances in dijet systems containing jets tagged as $W$ or $Z$ boson decays in pp collisions at $\sqrt{s}=8$ TeV, JHEP 08 (2014) 173 [arXiv: 1405.1994] [INSPIRE].

[33] CMS collaboration, The CMS experiment at the CERN LHC, 2008 JINST 3 S08004 [INSPIRE].

[34] J. Alwall, M. Herquet, F. Maltoni, O. Mattelaer and T. Stelzer, MadGraph 5: going beyond, JHEP 06 (2011) 128 [arXiv: 1106.0522] [INSPIRE].

[35] P. Nason, A new method for combining NLO QCD with shower Monte Carlo algorithms, JHEP 11 (2004) 040 [hep-ph/0409146] [INSPIRE].

[36] S. Frixione, P. Nason and C. Oleari, Matching NLO QCD computations with parton shower simulations: the POWHEG method, JHEP 11 (2007) 070 [arXiv:0709.2092] [INSPIRE].

[37] S. Alioli, P. Nason, C. Oleari and E. Re, A general framework for implementing NLO calculations in shower Monte Carlo programs: the POWHEG BOX, JHEP 06 (2010) 043 [arXiv: 1002.2581] [INSPIRE].

[38] S. Alioli, P. Nason, C. Oleari and E. Re, NLO single-top production matched with shower in POWHEG: s- and t-channel contributions, JHEP 09 (2009) 111 [Erratum ibid. 1002 (2010) 011] [arXiv:0907.4076] [INSPIRE].

[39] E. Re, Single-top Wt-channel production matched with parton showers using the POWHEG method, Eur. Phys. J. C 71 (2011) 1547 [arXiv: 1009.2450] [INSPIRE].

[40] S. Alioli, S.-O. Moch and P. Uwer, Hadronic top-quark pair-production with one jet and parton showering, JHEP 01 (2012) 137 [arXiv:1110.5251] [INSPIRE].

[41] T. Sjöstrand, S. Mrenna and P.Z. Skands, PYTHIA 6.4 physics and manual, JHEP 05 (2006) 026 [hep-ph/0603175] [INSPIRE].

[42] CMS collaboration, Measurement of the underlying event activity at the LHC with $\sqrt{s}=7$ $\mathrm{TeV}$ and comparison with $\sqrt{s}=0.9 \mathrm{TeV}$, JHEP 09 (2011) 109 [arXiv:1107.0330] [INSPIRE]. 
[43] J. Pumplin et al., New generation of parton distributions with uncertainties from global QCD analysis, JHEP 07 (2002) 012 [hep-ph/0201195] [INSPIRE].

[44] H.-L. Lai et al., New parton distributions for collider physics, Phys. Rev. D 82 (2010) 074024 [arXiv: 1007.2241] [INSPIRE].

[45] GEANT4 collaboration, S. Agostinelli et al., GEANT4 - a simulation toolkit, Nucl. Instrum. Meth. A 506 (2003) 250 [INSPIRE].

[46] J.M. Campbell, R.K. Ellis and D.L. Rainwater, Next-to-leading order QCD predictions for $W+2$ jet and $Z+2$ jet production at the CERN LHC, Phys. Rev. D 68 (2003) 094021 [hep-ph/0308195] [INSPIRE].

[47] J.M. Campbell, R.K. Ellis and C. Williams, Vector boson pair production at the LHC, JHEP 07 (2011) 018 [arXiv: 1105.0020] [INSPIRE].

[48] J.M. Campbell and R.K. Ellis, Top-quark processes at NLO in production and decay, arXiv:1204.1513 [INSPIRE].

[49] J.M. Campbell, R.K. Ellis and F. Tramontano, Single top production and decay at next-to-leading order, Phys. Rev. D 70 (2004) 094012 [hep-ph/0408158] [INSPIRE].

[50] Y. Li and F. Petriello, Combining QCD and electroweak corrections to dilepton production in FEWZ, Phys. Rev. D 86 (2012) 094034 [arXiv: 1208.5967] [INSPIRE].

[51] Y. Gao, A.V. Gritsan, Z. Guo, K. Melnikov, M. Schulze et al., Spin determination of single-produced resonances at hadron colliders, Phys. Rev. D 81 (2010) 075022 [arXiv:1001.3396] [INSPIRE].

[52] A. Belyaev, N.D. Christensen and A. Pukhov, CalcHEP 3.4 for collider physics within and beyond the standard model, Comput. Phys. Commun. 184 (2013) 1729 [arXiv:1207.6082] [INSPIRE].

[53] CMS collaboration, Tracking and primary vertex results in first $7 \mathrm{TeV}$ collisions, CMS-PAS-TRK-10-005 (2010).

[54] CMS collaboration, Performance of CMS muon reconstruction in pp collision events at $\sqrt{s}=7 \mathrm{TeV}, 2012$ JINST 7 P10002 [arXiv:1206.4071] [INSPIRE].

[55] CMS collaboration, Measurements of Inclusive $W$ and $Z$ Cross Sections in pp Collisions at $\sqrt{s}=7 \mathrm{TeV}, J H E P 01$ (2011) 080 [arXiv:1012.2466] [INSPIRE].

[56] CMS collaboration, Energy calibration and resolution of the CMS electromagnetic calorimeter in pp collisions at $\sqrt{s}=7$ TeV, 2013 JINST 8 P09009 [arXiv:1306.2016] [INSPIRE].

[57] CMS collaboration, Search for leptonic decays of $W^{\prime}$ bosons in pp collisions at $\sqrt{s}=7 \mathrm{TeV}$, JHEP 08 (2012) 023 [arXiv: 1204.4764] [INSPIRE].

[58] CMS collaboration, Particle-flow event reconstruction in CMS and performance for jets, taus and MET, CMS-PAS-PFT-09-001 (2009).

[59] CMS collaboration, Commissioning of the particle-flow event reconstruction with the first LHC collisions recorded in the CMS detector, CMS-PAS-PFT-10-001 (2010).

[60] M. Cacciari, G.P. Salam and G. Soyez, FastJet user manual, Eur. Phys. J. C 72 (2012) 1896 [arXiv: 1111.6097] [INSPIRE].

[61] M. Wobisch and T. Wengler, Hadronization corrections to jet cross-sections in deep inelastic scattering, hep-ph/9907280 [INSPIRE]. 
[62] M. Cacciari, G.P. Salam and G. Soyez, The anti- $k_{t}$ jet clustering algorithm, JHEP 04 (2008) 063 [arXiv: 0802.1189] [INSPIRE].

[63] CMS collaboration, Identification of b-quark jets with the CMS experiment, 2013 JINST 8 P04013 [arXiv: 1211.4462] [INSPIRE].

[64] CMS collaboration, Determination of jet energy calibration and transverse momentum resolution in CMS, 2011 JINST 6 P11002 [arXiv:1107.4277] [INSPIRE].

[65] CMS collaboration, Missing transverse energy performance of the CMS detector, 2011 JINST 6 P09001 [arXiv:1106.5048] [INSPIRE].

[66] CMS collaboration, MET performance in 8 TeV data, CMS-PAS-JME-12-002 (2012).

[67] Particle Data Group, J. Beringer et al., Review of particle physics, Phys. Rev. D 86 (2012) 010001 [INSPIRE].

[68] CMS collaboration, Studies of jet mass in dijet and W/Z + jet events, JHEP 05 (2013) 090 [arXiv: 1303.4811] [INSPIRE].

[69] S.D. Ellis, C.K. Vermilion and J.R. Walsh, Techniques for improved heavy particle searches with jet substructure, Phys. Rev. D 80 (2009) 051501 [arXiv:0903.5081] [INSPIRE].

[70] S.D. Ellis, C.K. Vermilion and J.R. Walsh, Recombination algorithms and jet substructure: pruning as a tool for heavy particle searches, Phys. Rev. D 81 (2010) 094023 [arXiv:0912.0033] [INSPIRE].

[71] J. Thaler and K. Van Tilburg, Identifying boosted objects with $N$-subjettiness, JHEP 03 (2011) 015 [arXiv: 1011.2268] [INSPIRE].

[72] S. Catani, Y.L. Dokshitzer, M.H. Seymour and B.R. Webber, Longitudinally invariant $K_{t}$ clustering algorithms for hadron hadron collisions, Nucl. Phys. B 406 (1993) 187 [INSPIRE].

[73] S.D. Ellis and D.E. Soper, Successive combination jet algorithm for hadron collisions, Phys. Rev. D 48 (1993) 3160 [hep-ph/9305266] [INSPIRE].

[74] CMS collaboration, Measurement of the properties of a Higgs boson in the four-lepton final state, Phys. Rev. D 89 (2014) 092007 [arXiv:1312.5353] [INSPIRE].

[75] F. Garwood, Fiducial Limits for the Poisson distribution, Biometrika 28 (1936) 437.

[76] M.J. Oreglia, A study of the reactions $\psi^{\prime} \rightarrow \gamma \gamma \psi$, Ph.D. thesis, Stanford University, Stanford, U.S.A. (1980), SLAC-R-236.

[77] CMS collaboration, Measurement of $W^{+} W^{-}$and $Z Z$ production cross sections in $p p$ collisions at $\sqrt{s}=8 \mathrm{TeV}$, Phys. Lett. B 721 (2013) 190 [arXiv:1301.4698] [InSPIRE].

[78] CMS collaboration, Measurement of the inelastic proton-proton cross section at $\sqrt{s}=7 \mathrm{TeV}$, Phys. Lett. B 722 (2013) 5 [arXiv:1210.6718] [INSPIRE].

[79] M. Botje et al., The PDF4LHC working group interim recommendations, arXiv:1101.0538 [INSPIRE].

[80] A.D. Martin, W.J. Stirling, R.S. Thorne and G. Watt, Parton distributions for the LHC, Eur. Phys. J. C 63 (2009) 189 [arXiv:0901.0002] [InSPIRE].

[81] R.D. Ball et al., Impact of heavy quark masses on parton distributions and LHC phenomenology, Nucl. Phys. B 849 (2011) 296 [arXiv:1101.1300] [INSPIRE].

[82] CMS collaboration, CMS luminosity based on pixel cluster counting - Summer 2013 update, CMS-PAS-LUM-13-001 (2013). 
[83] A.L. Read, Presentation of search results: the $C L_{s}$ technique, J. Phys. G 28 (2002) 2693 [INSPIRE].

[84] T. Junk, Confidence level computation for combining searches with small statistics, Nucl. Instrum. Meth. A 434 (1999) 435 [hep-ex/9902006] [INSPIRE].

[85] CMS collaboration, Search for narrow resonances using the dijet mass spectrum in pp collisions at $\sqrt{s}=8 \mathrm{TeV}$, Phys. Rev. D 87 (2013) 114015 [arXiv:1302.4794] [INSPIRE].

[86] G. Cowan, K. Cranmer, E. Gross and O. Vitells, Asymptotic formulae for likelihood-based tests of new physics, Eur. Phys. J. C 71 (2011) 1554 [arXiv:1007.1727] [INSPIRE]. 


\section{The CMS collaboration}

\section{Yerevan Physics Institute, Yerevan, Armenia}

V. Khachatryan, A.M. Sirunyan, A. Tumasyan

\section{Institut für Hochenergiephysik der OeAW, Wien, Austria}

W. Adam, T. Bergauer, M. Dragicevic, J. Erö, C. Fabjan ${ }^{1}$, M. Friedl, R. Frühwirth ${ }^{1}$, V.M. Ghete, C. Hartl, N. Hörmann, J. Hrubec, M. Jeitler ${ }^{1}$, W. Kiesenhofer, V. Knünz, M. Krammer ${ }^{1}$, I. Krätschmer, D. Liko, I. Mikulec, D. Rabady² , B. Rahbaran, H. Rohringer, R. Schöfbeck, J. Strauss, A. Taurok, W. Treberer-Treberspurg, W. Waltenberger, C.E. Wulz ${ }^{1}$

\section{National Centre for Particle and High Energy Physics, Minsk, Belarus}

V. Mossolov, N. Shumeiko, J. Suarez Gonzalez

\section{Universiteit Antwerpen, Antwerpen, Belgium}

S. Alderweireldt, M. Bansal, S. Bansal, T. Cornelis, E.A. De Wolf, X. Janssen, A. Knutsson, S. Luyckx, S. Ochesanu, B. Roland, R. Rougny, M. Van De Klundert, H. Van Haevermaet, P. Van Mechelen, N. Van Remortel, A. Van Spilbeeck

\section{Vrije Universiteit Brussel, Brussel, Belgium}

F. Blekman, S. Blyweert, J. D'Hondt, N. Daci, N. Heracleous, A. Kalogeropoulos, J. Keaveney, T.J. Kim, S. Lowette, M. Maes, A. Olbrechts, Q. Python, D. Strom, S. Tavernier, W. Van Doninck, P. Van Mulders, G.P. Van Onsem, I. Villella

\section{Université Libre de Bruxelles, Bruxelles, Belgium}

C. Caillol, B. Clerbaux, G. De Lentdecker, D. Dobur, L. Favart, A.P.R. Gay, A. Grebenyuk, A. Léonard, A. Mohammadi, L. Perniè ${ }^{2}$, T. Reis, T. Seva, L. Thomas, C. Vander Velde, P. Vanlaer, J. Wang

\section{Ghent University, Ghent, Belgium}

V. Adler, K. Beernaert, L. Benucci, A. Cimmino, S. Costantini, S. Crucy, S. Dildick, A. Fagot, G. Garcia, B. Klein, J. Mccartin, A.A. Ocampo Rios, D. Ryckbosch, S. Salva Diblen, M. Sigamani, N. Strobbe, F. Thyssen, M. Tytgat, E. Yazgan, N. Zaganidis

\section{Université Catholique de Louvain, Louvain-la-Neuve, Belgium}

S. Basegmez, C. Beluffi ${ }^{3}$, G. Bruno, R. Castello, A. Caudron, L. Ceard, G.G. Da Silveira, C. Delaere, T. du Pree, D. Favart, L. Forthomme, A. Giammanco ${ }^{4}$, J. Hollar, P. Jez, M. Komm, V. Lemaitre, J. Liao, C. Nuttens, D. Pagano, A. Pin, K. Piotrzkowski, A. Popov ${ }^{5}$, L. Quertenmont, M. Selvaggi, M. Vidal Marono, J.M. Vizan Garcia

\section{Université de Mons, Mons, Belgium}

N. Beliy, T. Caebergs, E. Daubie, G.H. Hammad

Centro Brasileiro de Pesquisas Fisicas, Rio de Janeiro, Brazil

G.A. Alves, M. Correa Martins Junior, T. Dos Reis Martins, M.E. Pol 
Universidade do Estado do Rio de Janeiro, Rio de Janeiro, Brazil

W.L. Aldá Júnior, W. Carvalho, J. Chinellato ${ }^{6}$, A. Custódio, E.M. Da Costa, D. De Jesus Damiao, C. De Oliveira Martins, S. Fonseca De Souza, H. Malbouisson, M. Malek, D. Matos Figueiredo, L. Mundim, H. Nogima, W.L. Prado Da Silva, J. Santaolalla, A. Santoro, A. Sznajder, E.J. Tonelli Manganote ${ }^{6}$, A. Vilela Pereira

Universidade Estadual Paulista $^{a}$, Universidade Federal do $\mathrm{ABC}^{b}$, São Paulo, Brazil

C.A. Bernardes ${ }^{b}$, F.A. Dias ${ }^{a, 7}$, T.R. Fernandez Perez Tomei ${ }^{a}$, E.M. Gregores ${ }^{b}$, P.G. Mercadante ${ }^{b}$, S.F. Novaes ${ }^{a}$, Sandra S. Padula ${ }^{a}$

Institute for Nuclear Research and Nuclear Energy, Sofia, Bulgaria

A. Aleksandrov, V. Genchev², P. Iaydjiev, A. Marinov, S. Piperov, M. Rodozov, G. Sultanov, M. Vutova

University of Sofia, Sofia, Bulgaria

A. Dimitrov, I. Glushkov, R. Hadjiiska, V. Kozhuharov, L. Litov, B. Pavlov, P. Petkov

Institute of High Energy Physics, Beijing, China

J.G. Bian, G.M. Chen, H.S. Chen, M. Chen, R. Du, C.H. Jiang, D. Liang, S. Liang, R. Plestina ${ }^{8}$, J. Tao, X. Wang, Z. Wang

State Key Laboratory of Nuclear Physics and Technology, Peking University, Beijing, China

C. Asawatangtrakuldee, Y. Ban, Y. Guo, Q. Li, W. Li, S. Liu, Y. Mao, S.J. Qian, D. Wang,

L. Zhang, W. Zou

Universidad de Los Andes, Bogota, Colombia

C. Avila, L.F. Chaparro Sierra, C. Florez, J.P. Gomez, B. Gomez Moreno, J.C. Sanabria

Technical University of Split, Split, Croatia

N. Godinovic, D. Lelas, D. Polic, I. Puljak

University of Split, Split, Croatia

Z. Antunovic, M. Kovac

Institute Rudjer Boskovic, Zagreb, Croatia

V. Brigljevic, K. Kadija, J. Luetic, D. Mekterovic, L. Sudic

University of Cyprus, Nicosia, Cyprus

A. Attikis, G. Mavromanolakis, J. Mousa, C. Nicolaou, F. Ptochos, P.A. Razis

Charles University, Prague, Czech Republic

M. Bodlak, M. Finger, M. Finger Jr.

Academy of Scientific Research and Technology of the Arab Republic of Egypt, Egyptian Network of High Energy Physics, Cairo, Egypt

Y. Assran ${ }^{9}$, S. Elgammal ${ }^{10}$, M.A. Mahmoud ${ }^{11}$, A. Radi ${ }^{10,12}$ 
National Institute of Chemical Physics and Biophysics, Tallinn, Estonia

M. Kadastik, M. Murumaa, M. Raidal, A. Tiko

Department of Physics, University of Helsinki, Helsinki, Finland

P. Eerola, G. Fedi, M. Voutilainen

Helsinki Institute of Physics, Helsinki, Finland

J. Härkönen, V. Karimäki, R. Kinnunen, M.J. Kortelainen, T. Lampén, K. Lassila-Perini,

S. Lehti, T. Lindén, P. Luukka, T. Mäenpää, T. Peltola, E. Tuominen, J. Tuominiemi,

E. Tuovinen, L. Wendland

Lappeenranta University of Technology, Lappeenranta, Finland

T. Tuuva

DSM/IRFU, CEA/Saclay, Gif-sur-Yvette, France

M. Besancon, F. Couderc, M. Dejardin, D. Denegri, B. Fabbro, J.L. Faure, C. Favaro,

F. Ferri, S. Ganjour, A. Givernaud, P. Gras, G. Hamel de Monchenault, P. Jarry, E. Locci,

J. Malcles, A. Nayak, J. Rander, A. Rosowsky, M. Titov

Laboratoire Leprince-Ringuet, Ecole Polytechnique, IN2P3-CNRS, Palaiseau, France

S. Baffioni, F. Beaudette, P. Busson, C. Charlot, T. Dahms, M. Dalchenko, L. Dobrzynski, N. Filipovic, A. Florent, R. Granier de Cassagnac, L. Mastrolorenzo, P. Miné, C. Mironov, I.N. Naranjo, M. Nguyen, C. Ochando, P. Paganini, R. Salerno, J.B. Sauvan, Y. Sirois, C. Veelken, Y. Yilmaz, A. Zabi

Institut Pluridisciplinaire Hubert Curien, Université de Strasbourg, Université de Haute Alsace Mulhouse, CNRS/IN2P3, Strasbourg, France

J.-L. Agram ${ }^{13}$, J. Andrea, A. Aubin, D. Bloch, J.-M. Brom, E.C. Chabert, C. Collard, E. Conte ${ }^{13}$, J.-C. Fontaine ${ }^{13}$, D. Gelé, U. Goerlach, C. Goetzmann, A.-C. Le Bihan, P. Van Hove

Centre de Calcul de l'Institut National de Physique Nucleaire et de Physique des Particules, CNRS/IN2P3, Villeurbanne, France

S. Gadrat

Université de Lyon, Université Claude Bernard Lyon 1, CNRS-IN2P3, Institut de Physique Nucléaire de Lyon, Villeurbanne, France

S. Beauceron, N. Beaupere, G. Boudoul ${ }^{2}$, S. Brochet, C.A. Carrillo Montoya, A. Carvalho Antunes De Oliveira, J. Chasserat, R. Chierici, D. Contardo ${ }^{2}$, P. Depasse, H. El Mamouni, J. Fan, J. Fay, S. Gascon, M. Gouzevitch, B. Ille, T. Kurca, M. Lethuillier, L. Mirabito, S. Perries, J.D. Ruiz Alvarez, D. Sabes, L. Sgandurra, V. Sordini, M. Vander Donckt, P. Verdier, S. Viret, H. Xiao

Institute of High Energy Physics and Informatization, Tbilisi State University, Tbilisi, Georgia

Z. Tsamalaidze ${ }^{14}$ 


\section{RWTH Aachen University, I. Physikalisches Institut, Aachen, Germany}

C. Autermann, S. Beranek, M. Bontenackels, B. Calpas, M. Edelhoff, L. Feld, O. Hindrichs, K. Klein, A. Ostapchuk, A. Perieanu, F. Raupach, J. Sammet, S. Schael, D. Sprenger, H. Weber, B. Wittmer, V. Zhukov ${ }^{5}$

\section{RWTH Aachen University, III. Physikalisches Institut A, Aachen, Germany}

M. Ata, J. Caudron, E. Dietz-Laursonn, D. Duchardt, M. Erdmann, R. Fischer, A. Güth, T. Hebbeker, C. Heidemann, K. Hoepfner, D. Klingebiel, S. Knutzen, P. Kreuzer, M. Merschmeyer, A. Meyer, M. Olschewski, K. Padeken, P. Papacz, H. Reithler, S.A. Schmitz, L. Sonnenschein, D. Teyssier, S. Thüer, M. Weber

\section{RWTH Aachen University, III. Physikalisches Institut B, Aachen, Germany}

V. Cherepanov, Y. Erdogan, G. Flügge, H. Geenen, M. Geisler, W. Haj Ahmad, F. Hoehle, B. Kargoll, T. Kress, Y. Kuessel, J. Lingemann², A. Nowack, I.M. Nugent, L. Perchalla, O. Pooth, A. Stahl

\section{Deutsches Elektronen-Synchrotron, Hamburg, Germany}

I. Asin, N. Bartosik, J. Behr, W. Behrenhoff, U. Behrens, A.J. Bell, M. Bergholz ${ }^{15}$, A. Bethani, K. Borras, A. Burgmeier, A. Cakir, L. Calligaris, A. Campbell, S. Choudhury, F. Costanza, C. Diez Pardos, S. Dooling, T. Dorland, G. Eckerlin, D. Eckstein, T. Eichhorn, G. Flucke, J. Garay Garcia, A. Geiser, P. Gunnellini, J. Hauk, G. Hellwig, M. Hempel, D. Horton, H. Jung, M. Kasemann, P. Katsas, J. Kieseler, C. Kleinwort, D. Krücker, W. Lange, J. Leonard, K. Lipka, A. Lobanov, W. Lohmann ${ }^{15}$, B. Lutz, R. Mankel, I. Marfin, I.-A. Melzer-Pellmann, A.B. Meyer, J. Mnich, A. Mussgiller, S. Naumann-Emme, O. Novgorodova, F. Nowak, E. Ntomari, H. Perrey, D. Pitzl, R. Placakyte, A. Raspereza, P.M. Ribeiro Cipriano, E. Ron, M.Ö. Sahin, J. Salfeld-Nebgen, P. Saxena, R. Schmidt ${ }^{15}$, T. Schoerner-Sadenius, M. Schröder, S. Spannagel, A.D.R. Vargas Trevino, R. Walsh, C. Wissing

\section{University of Hamburg, Hamburg, Germany}

M. Aldaya Martin, V. Blobel, M. Centis Vignali, J. Erfle, E. Garutti, K. Goebel, M. Görner, M. Gosselink, J. Haller, R.S. Höing, H. Kirschenmann, R. Klanner, R. Kogler, J. Lange, T. Lapsien, T. Lenz, I. Marchesini, J. Ott, T. Peiffer, N. Pietsch, D. Rathjens, C. Sander, H. Schettler, P. Schleper, E. Schlieckau, A. Schmidt, M. Seidel, J. Sibille ${ }^{16}$, V. Sola, H. Stadie, G. Steinbrück, D. Troendle, E. Usai, L. Vanelderen

\section{Institut für Experimentelle Kernphysik, Karlsruhe, Germany}

C. Barth, C. Baus, J. Berger, C. Böser, E. Butz, T. Chwalek, W. De Boer, A. Descroix, A. Dierlamm, M. Feindt, F. Hartmann², T. Hauth², U. Husemann, I. Katkov ${ }^{5}$, A. Kornmayer ${ }^{2}$, E. Kuznetsova, P. Lobelle Pardo, M.U. Mozer, Th. Müller, A. Nürnberg, G. Quast, K. Rabbertz, F. Ratnikov, S. Röcker, H.J. Simonis, F.M. Stober, R. Ulrich, J. Wagner-Kuhr, S. Wayand, T. Weiler, R. Wolf 
Institute of Nuclear and Particle Physics (INPP), NCSR Demokritos, Aghia Paraskevi, Greece

G. Anagnostou, G. Daskalakis, T. Geralis, V.A. Giakoumopoulou, A. Kyriakis, D. Loukas, A. Markou, C. Markou, A. Psallidas, I. Topsis-Giotis

University of Athens, Athens, Greece

L. Gouskos, A. Panagiotou, N. Saoulidou, E. Stiliaris

University of Ioánnina, Ioánnina, Greece

X. Aslanoglou, I. Evangelou, G. Flouris, C. Foudas, P. Kokkas, N. Manthos, I. Papadopoulos, E. Paradas

Wigner Research Centre for Physics, Budapest, Hungary

G. Bencze, C. Hajdu, P. Hidas, D. Horvath ${ }^{17}$, F. Sikler, V. Veszpremi, G. Vesztergombi ${ }^{18}$, A.J. Zsigmond

Institute of Nuclear Research ATOMKI, Debrecen, Hungary

N. Beni, S. Czellar, J. Karancsi ${ }^{19}$, J. Molnar, J. Palinkas, Z. Szillasi

University of Debrecen, Debrecen, Hungary

P. Raics, Z.L. Trocsanyi, B. Ujvari

National Institute of Science Education and Research, Bhubaneswar, India

S.K. Swain

Panjab University, Chandigarh, India

S.B. Beri, V. Bhatnagar, N. Dhingra, R. Gupta, A.K. Kalsi, M. Kaur, M. Mittal, N. Nishu, J.B. Singh

University of Delhi, Delhi, India

Ashok Kumar, Arun Kumar, S. Ahuja, A. Bhardwaj, B.C. Choudhary, A. Kumar, S. Malhotra, M. Naimuddin, K. Ranjan, V. Sharma

Saha Institute of Nuclear Physics, Kolkata, India

S. Banerjee, S. Bhattacharya, K. Chatterjee, S. Dutta, B. Gomber, Sa. Jain, Sh. Jain, R. Khurana, A. Modak, S. Mukherjee, D. Roy, S. Sarkar, M. Sharan

Bhabha Atomic Research Centre, Mumbai, India

A. Abdulsalam, D. Dutta, S. Kailas, V. Kumar, A.K. Mohanty², L.M. Pant, P. Shukla, A. Topkar

Tata Institute of Fundamental Research - EHEP, Mumbai, India

T. Aziz, R.M. Chatterjee, S. Ganguly, S. Ghosh, M. Guchait ${ }^{20}$, A. Gurtu ${ }^{21}$, G. Kole, S. Kumar, M. Maity ${ }^{22}$, G. Majumder, K. Mazumdar, G.B. Mohanty, B. Parida, K. Sudhakar, N. Wickramage ${ }^{23}$

Tata Institute of Fundamental Research - HECR, Mumbai, India

S. Banerjee, R.K. Dewanjee, S. Dugad 
Institute for Research in Fundamental Sciences (IPM), Tehran, Iran

H. Bakhshiansohi, H. Behnamian, S.M. Etesami ${ }^{24}$, A. Fahim ${ }^{25}$, R. Goldouzian, A. Jafari, M. Khakzad, M. Mohammadi Najafabadi, M. Naseri, S. Paktinat Mehdiabadi, B. Safarzadeh ${ }^{26}$, M. Zeinali

University College Dublin, Dublin, Ireland

M. Felcini, M. Grunewald

INFN Sezione di Bari ${ }^{a}$, Università di Bari ${ }^{b}$, Politecnico di Bari ${ }^{c}$, Bari, Italy M. Abbrescia ${ }^{a, b}$, L. Barbone $^{a, b}$, C. Calabria ${ }^{a, b}$, S.S. Chhibra ${ }^{a, b}$, A. Colaleo $^{a}$, D. Creanza $^{a, c}$, N. De Filippis ${ }^{a, c}$, M. De Palma ${ }^{a, b}$, L. Fiore ${ }^{a}$, G. Iaselli ${ }^{a, c}$, G. Maggi ${ }^{a, c}$, M. Maggi $^{a}, \mathrm{~S} \mathrm{My}^{a, c}$, S. Nuzzo ${ }^{a, b}$, N. Pacifico ${ }^{a}$, A. Pompili ${ }^{a, b}$, G. Pugliese ${ }^{a, c}$, R. Radogna $^{a, b, 2}$, G. Selvaggi ${ }^{a, b}$, L. Silvestris ${ }^{a, 2}$, G. Singh ${ }^{a, b}$, R. Venditti ${ }^{a, b}$, P. Verwilligen ${ }^{a}$, G. Zito ${ }^{a}$

INFN Sezione di Bologna ${ }^{a}$, Università di Bologna ${ }^{b}$, Bologna, Italy

G. Abbiendi ${ }^{a}$, A.C. Benvenuti ${ }^{a}$, D. Bonacorsi ${ }^{a}, b$, S. Braibant-Giacomelli ${ }^{a}, b$, L. Brigliadori ${ }^{a, b}$, R. Campanini ${ }^{a, b}$, P. Capiluppi ${ }^{a}, b$ A. Castro ${ }^{a, b}$, F.R. Cavallo ${ }^{a}$,

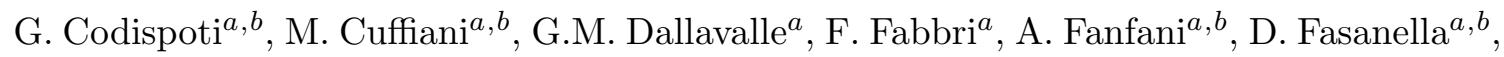
P. Giacomelli ${ }^{a}$, C. Grandi ${ }^{a}$, L. Guiducci ${ }^{a}, b$, S. Marcellini ${ }^{a}$, G. Masetti ${ }^{a, 2}$, A. Montanari ${ }^{a}$,

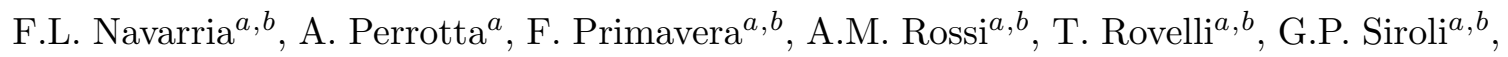
N. Tosi ${ }^{a, b}$, R. Travaglini ${ }^{a, b}$

INFN Sezione di Catania ${ }^{a}$, Università di Catania ${ }^{b}, \operatorname{CSFNSM}^{c}$, Catania, Italy

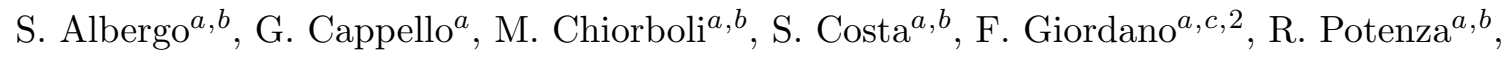
A. Tricomi ${ }^{a, b}$, C. Tuve ${ }^{a, b}$

INFN Sezione di Firenze ${ }^{a}$, Università di Firenze ${ }^{b}$, Firenze, Italy

G. Barbagli ${ }^{a}$, V. Ciulli ${ }^{a, b}$, C. Civinini ${ }^{a}$, R. D’Alessandro ${ }^{a, b}$, E. Focardi ${ }^{a, b}$, E. Gallo ${ }^{a}$, S. Gonzi ${ }^{a, b}$, V. Gori ${ }^{a, b, 2}$, P. Lenzi ${ }^{a, b}$, M. Meschini ${ }^{a}$, S. Paoletti ${ }^{a}$, G. Sguazzoni ${ }^{a}$, A. Tropiano ${ }^{a, b}$

INFN Laboratori Nazionali di Frascati, Frascati, Italy

L. Benussi, S. Bianco, F. Fabbri, D. Piccolo

INFN Sezione di Genova ${ }^{a}$, Università di Genova ${ }^{b}$, Genova, Italy

F. Ferro ${ }^{a}$, M. Lo Vetere ${ }^{a, b}$, E. Robutti ${ }^{a}$, S. Tosi ${ }^{a, b}$

INFN Sezione di Milano-Bicocca ${ }^{a}$, Università di Milano-Bicocca ${ }^{b}$, Milano, Italy

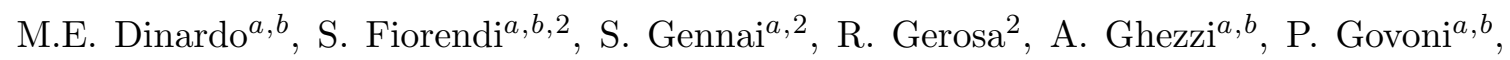
M.T. Lucchini ${ }^{a, b, 2}$, S. Malvezzi ${ }^{a}$, R.A. Manzoni ${ }^{a, b}$, A. Martelli ${ }^{a}, b$, B. Marzocchi, D. Menasce ${ }^{a}$, L. Moroni ${ }^{a}$, M. Paganoni ${ }^{a, b}$, D. Pedrini ${ }^{a}$, S. Ragazzi ${ }^{a}, b$, N. Redaelli ${ }^{a}$, T. Tabarelli de Fatis ${ }^{a, b}$

INFN Sezione di Napoli ${ }^{a}$, Università di Napoli 'Federico II' ${ }^{b}$, Università della Basilicata (Potenza) ${ }^{c}$, Università G. Marconi (Roma) ${ }^{d}$, Napoli, Italy

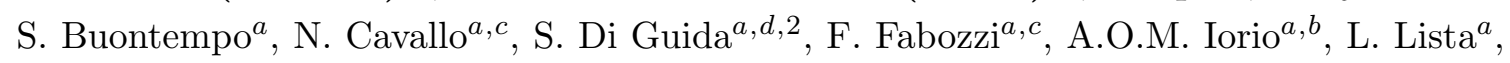
S. Meola ${ }^{a, d, 2}$, M. Merola ${ }^{a}$, P. Paolucci ${ }^{a, 2}$ 
INFN Sezione di Padova ${ }^{a}$, Università di Padova ${ }^{b}$, Università di Trento (Trento) ${ }^{c}$, Padova, Italy

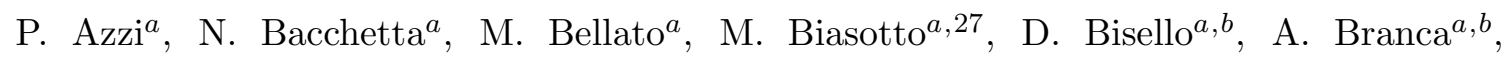
R. Carlin ${ }^{a, b}$, P. Checchia ${ }^{a}$, M. Dall'Osso ${ }^{a, b}$, T. Dorigo ${ }^{a}$, F. Fanzago ${ }^{a}$, M. Galanti ${ }^{a}, b$, F. Gasparini ${ }^{a, b}$, U. Gasparini ${ }^{a, b}$, A. Gozzelino ${ }^{a}$, K. Kanishchev ${ }^{a, c}$, S. Lacaprara ${ }^{a}$, M. Margoni ${ }^{a, b}$, A.T. Meneguzzo ${ }^{a, b}$, J. Pazzini ${ }^{a, b}$, N. Pozzobon ${ }^{a, b}$, P. Ronchese ${ }^{a, b}$,

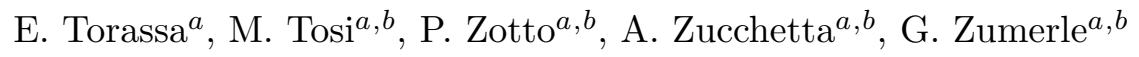

INFN Sezione di Pavia ${ }^{a}$, Università di Pavia ${ }^{b}$, Pavia, Italy

M. Gabusi ${ }^{a, b}$, S.P. Ratti ${ }^{a, b}$, C. Riccardi ${ }^{a, b}$, P. Salvini ${ }^{a}$, P. Vitulo ${ }^{a, b}$

INFN Sezione di Perugia ${ }^{a}$, Università di Perugia ${ }^{b}$, Perugia, Italy

M. Biasini ${ }^{a}, b$, G.M. Bilei ${ }^{a}$, D. Ciangottini ${ }^{a, b}$, L. Fanò ${ }^{a, b}$, P. Lariccia ${ }^{a, b}$, G. Mantovani $^{a, b}$,

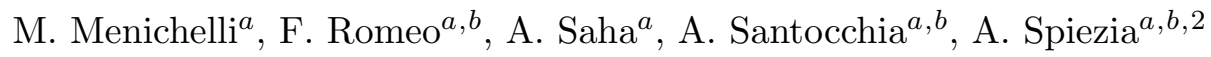

INFN Sezione di Pisa ${ }^{a}$, Università di Pisa ${ }^{b}$, Scuola Normale Superiore di Pisa ${ }^{c}$, Pisa, Italy

K. Androsov ${ }^{a, 28}$, P. Azzurri ${ }^{a}$, G. Bagliesi ${ }^{a}$, J. Bernardini ${ }^{a}$, T. Boccali ${ }^{a}$, G. Broccolo ${ }^{a, c}$, R. Castaldi ${ }^{a}$, M.A. Ciocci ${ }^{a, 28}$, R. Dell'Orso ${ }^{a}$, S. Donato ${ }^{a, c}$, F. Fiori ${ }^{a, c}$, L. Foà ${ }^{a, c}$,

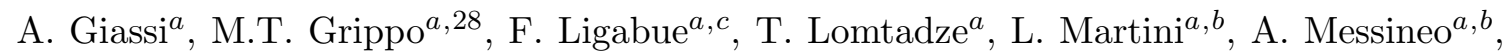
C.S. Moon ${ }^{a, 29}$, F. Palla ${ }^{a, 2}$, A. Rizzi ${ }^{a, b}$, A. Savoy-Navarro ${ }^{a, 30}$, A.T. Serban ${ }^{a}$, P. Spagnolo ${ }^{a}$,

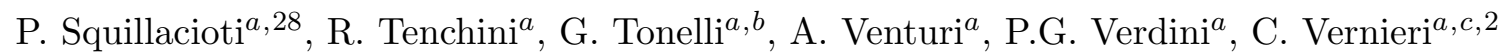

INFN Sezione di Roma ${ }^{a}$, Università di Roma ${ }^{b}$, Roma, Italy

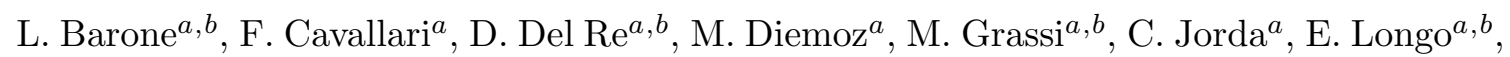
F. Margaroli ${ }^{a, b}$, P. Meridiani ${ }^{a}$, F. Micheli ${ }^{a, b, 2}$, S. Nourbakhsh ${ }^{a, b}$, G. Organtini ${ }^{a, b}$,

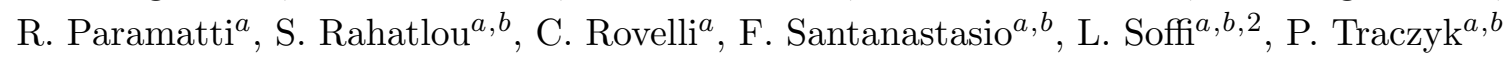

INFN Sezione di Torino ${ }^{a}$, Università di Torino ${ }^{b}$, Università del Piemonte Orientale (Novara) ${ }^{c}$, Torino, Italy

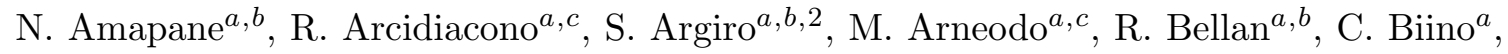

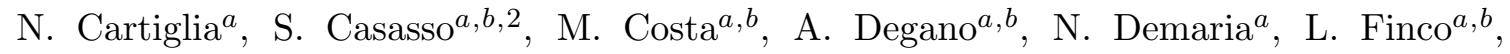

C. Mariotti ${ }^{a}$, S. Maselli ${ }^{a}$, E. Migliore ${ }^{a, b}$, V. Monaco ${ }^{a, b}$, M. Musich $^{a}$, M.M. Obertino $^{a, c, 2}$,

G. Ortona $^{a, b}$, L. Pacher ${ }^{a, b}$, N. Pastrone ${ }^{a}$, M. Pelliccioni ${ }^{a}$, G.L. Pinna Angioni ${ }^{a}, b$,

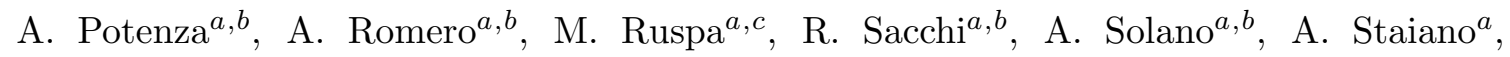

U. Tamponi ${ }^{a}$

INFN Sezione di Trieste ${ }^{a}$, Università di Trieste ${ }^{b}$, Trieste, Italy

S. Belforte ${ }^{a}$, V. Candelise ${ }^{a, b}$, M. Casarsa ${ }^{a}$, F. Cossutti ${ }^{a}$, G. Della Ricca ${ }^{a, b}$, B. Gobbo ${ }^{a}$,

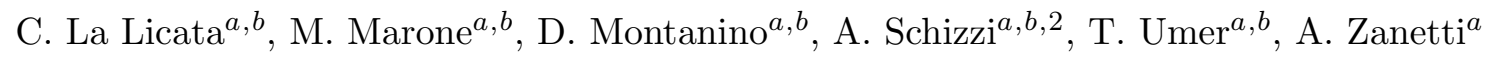

Kangwon National University, Chunchon, Korea

S. Chang, A. Kropivnitskaya, S.K. Nam

Kyungpook National University, Daegu, Korea

D.H. Kim, G.N. Kim, M.S. Kim, D.J. Kong, S. Lee, Y.D. Oh, H. Park, A. Sakharov, D.C. Son 
Chonnam National University, Institute for Universe and Elementary Particles, Kwangju, Korea

J.Y. Kim, S. Song

Korea University, Seoul, Korea

S. Choi, D. Gyun, B. Hong, M. Jo, H. Kim, Y. Kim, B. Lee, K.S. Lee, S.K. Park, Y. Roh

University of Seoul, Seoul, Korea

M. Choi, J.H. Kim, I.C. Park, S. Park, G. Ryu, M.S. Ryu

Sungkyunkwan University, Suwon, Korea

Y. Choi, Y.K. Choi, J. Goh, E. Kwon, J. Lee, H. Seo, I. Yu

Vilnius University, Vilnius, Lithuania

A. Juodagalvis

National Centre for Particle Physics, Universiti Malaya, Kuala Lumpur, Malaysia

J.R. Komaragiri

Centro de Investigacion y de Estudios Avanzados del IPN, Mexico City, Mexico

H. Castilla-Valdez, E. De La Cruz-Burelo, I. Heredia-de La Cruz ${ }^{31}$, R. Lopez-Fernandez,

A. Sanchez-Hernandez

Universidad Iberoamericana, Mexico City, Mexico

S. Carrillo Moreno, F. Vazquez Valencia

Benemerita Universidad Autonoma de Puebla, Puebla, Mexico

I. Pedraza, H.A. Salazar Ibarguen

Universidad Autónoma de San Luis Potosí, San Luis Potosí, Mexico

E. Casimiro Linares, A. Morelos Pineda

University of Auckland, Auckland, New Zealand

D. Krofcheck

University of Canterbury, Christchurch, New Zealand

P.H. Butler, S. Reucroft

National Centre for Physics, Quaid-I-Azam University, Islamabad, Pakistan

A. Ahmad, M. Ahmad, Q. Hassan, H.R. Hoorani, S. Khalid, W.A. Khan, T. Khurshid, M.A. Shah, M. Shoaib

National Centre for Nuclear Research, Swierk, Poland

H. Bialkowska, M. Bluj ${ }^{32}$, B. Boimska, T. Frueboes, M. Górski, M. Kazana, K. Nawrocki,

K. Romanowska-Rybinska, M. Szleper, P. Zalewski

Institute of Experimental Physics, Faculty of Physics, University of Warsaw, Warsaw, Poland

G. Brona, K. Bunkowski, M. Cwiok, W. Dominik, K. Doroba, A. Kalinowski, M. Konecki, J. Krolikowski, M. Misiura, M. Olszewski, W. Wolszczak 
Laboratório de Instrumentação e Física Experimental de Partículas, Lisboa, Portugal

P. Bargassa, C. Beirão Da Cruz E Silva, P. Faccioli, P.G. Ferreira Parracho, M. Gallinaro, F. Nguyen, J. Rodrigues Antunes, J. Seixas, J. Varela, P. Vischia

Joint Institute for Nuclear Research, Dubna, Russia

S. Afanasiev, P. Bunin, M. Gavrilenko, I. Golutvin, I. Gorbunov, A. Kamenev, V. Karjavin, V. Konoplyanikov, A. Lanev, A. Malakhov, V. Matveev ${ }^{33}$, P. Moisenz, V. Palichik, V. Perelygin, S. Shmatov, N. Skatchkov, V. Smirnov, A. Zarubin

Petersburg Nuclear Physics Institute, Gatchina (St. Petersburg), Russia

V. Golovtsov, Y. Ivanov, V. Kim ${ }^{34}$, P. Levchenko, V. Murzin, V. Oreshkin, I. Smirnov, V. Sulimov, L. Uvarov, S. Vavilov, A. Vorobyev, An. Vorobyev

Institute for Nuclear Research, Moscow, Russia

Yu. Andreev, A. Dermenev, S. Gninenko, N. Golubev, M. Kirsanov, N. Krasnikov, A. Pashenkov, D. Tlisov, A. Toropin

Institute for Theoretical and Experimental Physics, Moscow, Russia

V. Epshteyn, V. Gavrilov, N. Lychkovskaya, V. Popov, G. Safronov, S. Semenov, A. Spiridonov, V. Stolin, E. Vlasov, A. Zhokin

P.N. Lebedev Physical Institute, Moscow, Russia

V. Andreev, M. Azarkin, I. Dremin, M. Kirakosyan, A. Leonidov, G. Mesyats, S.V. Rusakov, A. Vinogradov

Skobeltsyn Institute of Nuclear Physics, Lomonosov Moscow State University, Moscow, Russia

A. Belyaev, E. Boos, M. Dubinin 7 , L. Dudko, A. Ershov, A. Gribushin, V. Klyukhin, O. Kodolova, I. Lokhtin, S. Obraztsov, M. Perfilov, S. Petrushanko, V. Savrin

State Research Center of Russian Federation, Institute for High Energy Physics, Protvino, Russia

I. Azhgirey, I. Bayshev, S. Bitioukov, V. Kachanov, A. Kalinin, D. Konstantinov, V. Krychkine, V. Petrov, R. Ryutin, A. Sobol, L. Tourtchanovitch, S. Troshin, N. Tyurin, A. Uzunian, A. Volkov

University of Belgrade, Faculty of Physics and Vinca Institute of Nuclear Sciences, Belgrade, Serbia

P. Adzic ${ }^{35}$, M. Dordevic, M. Ekmedzic, J. Milosevic

Centro de Investigaciones Energéticas Medioambientales y Tecnológicas (CIEMAT), Madrid, Spain

J. Alcaraz Maestre, C. Battilana, E. Calvo, M. Cerrada, M. Chamizo Llatas ${ }^{2}$, N. Colino, B. De La Cruz, A. Delgado Peris, D. Domínguez Vázquez, A. Escalante Del Valle, C. Fernandez Bedoya, J.P. Fernández Ramos, J. Flix, M.C. Fouz, P. Garcia-Abia, O. Gonzalez Lopez, S. Goy Lopez, J.M. Hernandez, M.I. Josa, G. Merino, E. Navarro De Martino, 
A. Pérez-Calero Yzquierdo, J. Puerta Pelayo, A. Quintario Olmeda, I. Redondo, L. Romero, M.S. Soares

\section{Universidad Autónoma de Madrid, Madrid, Spain}

C. Albajar, J.F. de Trocóniz, M. Missiroli

\section{Universidad de Oviedo, Oviedo, Spain}

H. Brun, J. Cuevas, J. Fernandez Menendez, S. Folgueras, I. Gonzalez Caballero, L. Lloret Iglesias

Instituto de Física de Cantabria (IFCA), CSIC-Universidad de Cantabria, Santander, Spain

J.A. Brochero Cifuentes, I.J. Cabrillo, A. Calderon, J. Duarte Campderros, M. Fernandez, G. Gomez, A. Graziano, A. Lopez Virto, J. Marco, R. Marco, C. Martinez Rivero, F. Matorras, F.J. Munoz Sanchez, J. Piedra Gomez, T. Rodrigo, A.Y. Rodríguez-Marrero, A. Ruiz-Jimeno, L. Scodellaro, I. Vila, R. Vilar Cortabitarte

\section{CERN, European Organization for Nuclear Research, Geneva, Switzerland}

D. Abbaneo, E. Auffray, G. Auzinger, M. Bachtis, P. Baillon, A.H. Ball, D. Barney, A. Benaglia, J. Bendavid, L. Benhabib, J.F. Benitez, C. Bernet ${ }^{8}$, G. Bianchi, P. Bloch, A. Bocci, A. Bonato, O. Bondu, C. Botta, H. Breuker, T. Camporesi, G. Cerminara, T. Christiansen, S. Colafranceschi ${ }^{36}$, M. D'Alfonso, D. d'Enterria, A. Dabrowski, A. David, F. De Guio, A. De Roeck, S. De Visscher, M. Dobson, N. Dupont-Sagorin, A. Elliott-Peisert, J. Eugster, G. Franzoni, W. Funk, M. Giffels, D. Gigi, K. Gill, D. Giordano, M. Girone, F. Glege, R. Guida, S. Gundacker, M. Guthoff, J. Hammer, M. Hansen, P. Harris, J. Hegeman, V. Innocente, P. Janot, K. Kousouris, K. Krajczar, P. Lecoq, C. Lourenço, N. Magini, L. Malgeri, M. Mannelli, L. Masetti, F. Meijers, S. Mersi, E. Meschi, F. Moortgat, S. Morovic, M. Mulders, P. Musella, L. Orsini, L. Pape, E. Perez, L. Perrozzi, A. Petrilli, G. Petrucciani, A. Pfeiffer, M. Pierini, M. Pimiä, D. Piparo, M. Plagge, A. Racz, G. Rolandi ${ }^{37}$, M. Rovere, H. Sakulin, C. Schäfer, C. Schwick, S. Sekmen, A. Sharma, P. Siegrist, P. Silva, M. Simon, P. Sphicas ${ }^{38}$, D. Spiga, J. Steggemann, B. Stieger, M. Stoye, D. Treille, A. Tsirou, G.I. Veres ${ }^{18}$, J.R. Vlimant, N. Wardle, H.K. Wöhri, W.D. Zeuner

\section{Paul Scherrer Institut, Villigen, Switzerland}

W. Bertl, K. Deiters, W. Erdmann, R. Horisberger, Q. Ingram, H.C. Kaestli, S. König, D. Kotlinski, U. Langenegger, D. Renker, T. Rohe

\section{Institute for Particle Physics, ETH Zurich, Zurich, Switzerland}

F. Bachmair, L. Bäni, L. Bianchini, P. Bortignon, M.A. Buchmann, B. Casal, N. Chanon, A. Deisher, G. Dissertori, M. Dittmar, M. Donegà, M. Dünser, P. Eller, C. Grab, D. Hits, W. Lustermann, B. Mangano, A.C. Marini, P. Martinez Ruiz del Arbol, D. Meister, N. Mohr, C. Nägeli ${ }^{39}$, P. Nef, F. Nessi-Tedaldi, F. Pandolfi, F. Pauss, M. Peruzzi, M. Quittnat, L. Rebane, F.J. Ronga, M. Rossini, A. Starodumov ${ }^{40}$, M. Takahashi, K. Theofilatos, R. Wallny, H.A. Weber 
Universität Zürich, Zurich, Switzerland

C. Amsler ${ }^{41}$, M.F. Canelli, V. Chiochia, A. De Cosa, A. Hinzmann, T. Hreus, M. Ivova Rikova, B. Kilminster, B. Millan Mejias, J. Ngadiuba, P. Robmann, H. Snoek, S. Taroni, M. Verzetti, Y. Yang

National Central University, Chung-Li, Taiwan

M. Cardaci, K.H. Chen, C. Ferro, C.M. Kuo, W. Lin, Y.J. Lu, R. Volpe, S.S. Yu

National Taiwan University (NTU), Taipei, Taiwan

P. Chang, Y.H. Chang, Y.W. Chang, Y. Chao, K.F. Chen, P.H. Chen, C. Dietz, U. Grundler, W.-S. Hou, K.Y. Kao, Y.J. Lei, Y.F. Liu, R.-S. Lu, D. Majumder, E. Petrakou, X. Shi, Y.M. Tzeng, R. Wilken

Chulalongkorn University, Bangkok, Thailand

B. Asavapibhop, N. Srimanobhas, N. Suwonjandee

Cukurova University, Adana, Turkey

A. Adiguzel, M.N. Bakirci ${ }^{42}$, S. Cerci ${ }^{43}$, C. Dozen, I. Dumanoglu, E. Eskut, S. Girgis, G. Gokbulut, E. Gurpinar, I. Hos, E.E. Kangal, A. Kayis Topaksu, G. Onengut ${ }^{44}$, K. Ozdemir, S. Ozturk ${ }^{42}$, A. Polatoz, K. Sogut ${ }^{45}$, D. Sunar Cerci ${ }^{43}$, B. Tali ${ }^{43}$, H. Topakli² ${ }^{42}$, M. Vergili

Middle East Technical University, Physics Department, Ankara, Turkey

I.V. Akin, B. Bilin, S. Bilmis, H. Gamsizkan, G. Karapinar ${ }^{46}$, K. Ocalan, U.E. Surat, M. Yalvac, M. Zeyrek

Bogazici University, Istanbul, Turkey

E. Gülmez, B. Isildak ${ }^{47}$, M. Kaya ${ }^{48}$, O. Kaya ${ }^{48}$

Istanbul Technical University, Istanbul, Turkey

H. Bahtiyar ${ }^{49}$, E. Barlas, K. Cankocak, F.I. Vardarl,, M. Yücel

National Scientific Center, Kharkov Institute of Physics and Technology, Kharkov, Ukraine

L. Levchuk, P. Sorokin

\section{University of Bristol, Bristol, United Kingdom}

J.J. Brooke, E. Clement, D. Cussans, H. Flacher, R. Frazier, J. Goldstein, M. Grimes, G.P. Heath, H.F. Heath, J. Jacob, L. Kreczko, C. Lucas, Z. Meng, D.M. Newbold ${ }^{50}$, S. Paramesvaran, A. Poll, S. Senkin, V.J. Smith, T. Williams

\section{Rutherford Appleton Laboratory, Didcot, United Kingdom}

K.W. Bell, A. Belyaev ${ }^{51}$, C. Brew, R.M. Brown, D.J.A. Cockerill, J.A. Coughlan, K. Harder, S. Harper, E. Olaiya, D. Petyt, C.H. Shepherd-Themistocleous, A. Thea, I.R. Tomalin, W.J. Womersley, S.D. Worm

\section{Imperial College, London, United Kingdom}

M. Baber, R. Bainbridge, O. Buchmuller, D. Burton, D. Colling, N. Cripps, M. Cutajar, P. Dauncey, G. Davies, M. Della Negra, P. Dunne, W. Ferguson, J. Fulcher, D. Futyan, 
A. Gilbert, G. Hall, G. Iles, M. Jarvis, G. Karapostoli, M. Kenzie, R. Lane, R. Lucas ${ }^{50}$, L. Lyons, A.-M. Magnan, S. Malik, J. Marrouche, B. Mathias, J. Nash, A. Nikitenko ${ }^{40}$, J. Pela, M. Pesaresi, K. Petridis, D.M. Raymond, S. Rogerson, A. Rose, C. Seez, P. Sharp ${ }^{\dagger}$, A. Tapper, M. Vazquez Acosta, T. Virdee

\section{Brunel University, Uxbridge, United Kingdom}

J.E. Cole, P.R. Hobson, A. Khan, P. Kyberd, D. Leggat, D. Leslie, W. Martin, I.D. Reid, P. Symonds, L. Teodorescu, M. Turner

Baylor University, Waco, U.S.A.

J. Dittmann, K. Hatakeyama, A. Kasmi, H. Liu, T. Scarborough

The University of Alabama, Tuscaloosa, U.S.A.

O. Charaf, S.I. Cooper, C. Henderson, P. Rumerio

\section{Boston University, Boston, U.S.A.}

A. Avetisyan, T. Bose, C. Fantasia, A. Heister, P. Lawson, C. Richardson, J. Rohlf, D. Sperka, J. St. John, L. Sulak

\section{Brown University, Providence, U.S.A.}

J. Alimena, S. Bhattacharya, G. Christopher, D. Cutts, Z. Demiragli, A. Ferapontov, A. Garabedian, U. Heintz, S. Jabeen, G. Kukartsev, E. Laird, G. Landsberg, M. Luk, M. Narain, M. Segala, T. Sinthuprasith, T. Speer, J. Swanson

University of California, Davis, Davis, U.S.A.

R. Breedon, G. Breto, M. Calderon De La Barca Sanchez, S. Chauhan, M. Chertok, J. Conway, R. Conway, P.T. Cox, R. Erbacher, M. Gardner, W. Ko, R. Lander, T. Miceli, M. Mulhearn, D. Pellett, J. Pilot, F. Ricci-Tam, M. Searle, S. Shalhout, J. Smith, M. Squires, D. Stolp, M. Tripathi, S. Wilbur, R. Yohay

University of California, Los Angeles, U.S.A.

R. Cousins, P. Everaerts, C. Farrell, J. Hauser, M. Ignatenko, G. Rakness, E. Takasugi, V. Valuev, M. Weber

\section{University of California, Riverside, Riverside, U.S.A.}

J. Babb, R. Clare, J. Ellison, J.W. Gary, G. Hanson, J. Heilman, P. Jandir, E. Kennedy, F. Lacroix, H. Liu, O.R. Long, A. Luthra, M. Malberti, H. Nguyen, A. Shrinivas, J. Sturdy, S. Sumowidagdo, S. Wimpenny

\section{University of California, San Diego, La Jolla, U.S.A.}

W. Andrews, J.G. Branson, G.B. Cerati, S. Cittolin, R.T. D'Agnolo, D. Evans, A. Holzner, R. Kelley, M. Lebourgeois, J. Letts, I. Macneill, D. Olivito, S. Padhi, C. Palmer, M. Pieri, M. Sani, V. Sharma, S. Simon, E. Sudano, M. Tadel, Y. Tu, A. Vartak, F. Würthwein, A. Yagil, J. Yoo

University of California, Santa Barbara, Santa Barbara, U.S.A.

D. Barge, J. Bradmiller-Feld, C. Campagnari, T. Danielson, A. Dishaw, K. Flowers, M. Franco Sevilla, P. Geffert, C. George, F. Golf, J. Incandela, C. Justus, N. Mccoll, J. Richman, D. Stuart, W. To, C. West 
California Institute of Technology, Pasadena, U.S.A.

A. Apresyan, A. Bornheim, J. Bunn, Y. Chen, E. Di Marco, J. Duarte, A. Mott, H.B. Newman, C. Pena, C. Rogan, M. Spiropulu, V. Timciuc, R. Wilkinson, S. Xie, R.Y. Zhu

Carnegie Mellon University, Pittsburgh, U.S.A.

V. Azzolini, A. Calamba, R. Carroll, T. Ferguson, Y. Iiyama, M. Paulini, J. Russ, H. Vogel, I. Vorobiev

University of Colorado at Boulder, Boulder, U.S.A.

J.P. Cumalat, B.R. Drell, W.T. Ford, A. Gaz, E. Luiggi Lopez, U. Nauenberg, J.G. Smith, K. Stenson, K.A. Ulmer, S.R. Wagner

Cornell University, Ithaca, U.S.A.

J. Alexander, A. Chatterjee, J. Chu, S. Dittmer, N. Eggert, W. Hopkins, B. Kreis, N. Mirman, G. Nicolas Kaufman, J.R. Patterson, A. Ryd, E. Salvati, L. Skinnari, W. Sun, W.D. Teo, J. Thom, J. Thompson, J. Tucker, Y. Weng, L. Winstrom, P. Wittich

Fairfield University, Fairfield, U.S.A.

D. Winn

Fermi National Accelerator Laboratory, Batavia, U.S.A.

S. Abdullin, M. Albrow, J. Anderson, G. Apollinari, L.A.T. Bauerdick, A. Beretvas, J. Berryhill, P.C. Bhat, K. Burkett, J.N. Butler, H.W.K. Cheung, F. Chlebana, S. Cihangir, V.D. Elvira, I. Fisk, J. Freeman, E. Gottschalk, L. Gray, D. Green, S. Grünendahl, O. Gutsche, J. Hanlon, D. Hare, R.M. Harris, J. Hirschauer, B. Hooberman, S. Jindariani, M. Johnson, U. Joshi, K. Kaadze, B. Klima, S. Kwan, J. Linacre, D. Lincoln, R. Lipton, T. Liu, J. Lykken, K. Maeshima, J.M. Marraffino, V.I. Martinez Outschoorn, S. Maruyama, D. Mason, P. McBride, K. Mishra, S. Mrenna, Y. Musienko ${ }^{33}$, S. Nahn, C. NewmanHolmes, V. O’Dell, O. Prokofyev, E. Sexton-Kennedy, S. Sharma, A. Soha, W.J. Spalding, L. Spiegel, L. Taylor, S. Tkaczyk, N.V. Tran, L. Uplegger, E.W. Vaandering, R. Vidal, A. Whitbeck, J. Whitmore, F. Yang

University of Florida, Gainesville, U.S.A.

D. Acosta, P. Avery, D. Bourilkov, M. Carver, T. Cheng, D. Curry, S. Das, M. De Gruttola, G.P. Di Giovanni, R.D. Field, M. Fisher, I.K. Furic, J. Hugon, J. Konigsberg, A. Korytov, T. Kypreos, J.F. Low, K. Matchev, P. Milenovic ${ }^{52}$, G. Mitselmakher, L. Muniz, A. Rinkevicius, L. Shchutska, N. Skhirtladze, M. Snowball, J. Yelton, M. Zakaria

Florida International University, Miami, U.S.A.

V. Gaultney, S. Hewamanage, S. Linn, P. Markowitz, G. Martinez, J.L. Rodriguez

Florida State University, Tallahassee, U.S.A.

T. Adams, A. Askew, J. Bochenek, B. Diamond, J. Haas, S. Hagopian, V. Hagopian, K.F. Johnson, H. Prosper, V. Veeraraghavan, M. Weinberg

Florida Institute of Technology, Melbourne, U.S.A.

M.M. Baarmand, M. Hohlmann, H. Kalakhety, F. Yumiceva 
University of Illinois at Chicago (UIC), Chicago, U.S.A.

M.R. Adams, L. Apanasevich, V.E. Bazterra, D. Berry, R.R. Betts, I. Bucinskaite, R. Cavanaugh, O. Evdokimov, L. Gauthier, C.E. Gerber, D.J. Hofman, S. Khalatyan, P. Kurt, D.H. Moon, C. O'Brien, C. Silkworth, P. Turner, N. Varelas

The University of Iowa, Iowa City, U.S.A.

E.A. Albayrak ${ }^{49}$, B. Bilki ${ }^{53}$, W. Clarida, K. Dilsiz, F. Duru, M. Haytmyradov, J.-P. Merlo, H. Mermerkaya ${ }^{54}$, A. Mestvirishvili, A. Moeller, J. Nachtman, H. Ogul, Y. Onel, F. Ozok ${ }^{49}$, A. Penzo, R. Rahmat, S. Sen, P. Tan, E. Tiras, J. Wetzel, T. Yetkin ${ }^{55}$, K. Yi

Johns Hopkins University, Baltimore, U.S.A.

B.A. Barnett, B. Blumenfeld, S. Bolognesi, D. Fehling, A.V. Gritsan, P. Maksimovic, C. Martin, M. Swartz

The University of Kansas, Lawrence, U.S.A.

P. Baringer, A. Bean, G. Benelli, C. Bruner, J. Gray, R.P. Kenny III, M. Murray, D. Noonan, S. Sanders, J. Sekaric, R. Stringer, Q. Wang, J.S. Wood

Kansas State University, Manhattan, U.S.A.

A.F. Barfuss, I. Chakaberia, A. Ivanov, S. Khalil, M. Makouski, Y. Maravin, L.K. Saini, S. Shrestha, I. Svintradze

Lawrence Livermore National Laboratory, Livermore, U.S.A.

J. Gronberg, D. Lange, F. Rebassoo, D. Wright

University of Maryland, College Park, U.S.A.

A. Baden, B. Calvert, S.C. Eno, J.A. Gomez, N.J. Hadley, R.G. Kellogg, T. Kolberg, Y. Lu, M. Marionneau, A.C. Mignerey, K. Pedro, A. Skuja, M.B. Tonjes, S.C. Tonwar

Massachusetts Institute of Technology, Cambridge, U.S.A.

A. Apyan, R. Barbieri, G. Bauer, W. Busza, I.A. Cali, M. Chan, L. Di Matteo, V. Dutta, G. Gomez Ceballos, M. Goncharov, D. Gulhan, M. Klute, Y.S. Lai, Y.-J. Lee, A. Levin, P.D. Luckey, T. Ma, C. Paus, D. Ralph, C. Roland, G. Roland, G.S.F. Stephans, F. Stöckli, K. Sumorok, D. Velicanu, J. Veverka, B. Wyslouch, M. Yang, M. Zanetti, V. Zhukova

University of Minnesota, Minneapolis, U.S.A.

B. Dahmes, A. De Benedetti, A. Gude, S.C. Kao, K. Klapoetke, Y. Kubota, J. Mans,

N. Pastika, R. Rusack, A. Singovsky, N. Tambe, J. Turkewitz

University of Mississippi, Oxford, U.S.A.

J.G. Acosta, S. Oliveros

University of Nebraska-Lincoln, Lincoln, U.S.A.

E. Avdeeva, K. Bloom, S. Bose, D.R. Claes, A. Dominguez, R. Gonzalez Suarez, J. Keller, D. Knowlton, I. Kravchenko, J. Lazo-Flores, S. Malik, F. Meier, G.R. Snow

State University of New York at Buffalo, Buffalo, U.S.A.

J. Dolen, A. Godshalk, I. Iashvili, A. Kharchilava, A. Kumar, S. Rappoccio 
Northeastern University, Boston, U.S.A.

G. Alverson, E. Barberis, D. Baumgartel, M. Chasco, J. Haley, A. Massironi, D.M. Morse,

D. Nash, T. Orimoto, D. Trocino, D. Wood, J. Zhang

Northwestern University, Evanston, U.S.A.

K.A. Hahn, A. Kubik, N. Mucia, N. Odell, B. Pollack, A. Pozdnyakov, M. Schmitt, S. Stoynev, K. Sung, M. Velasco, S. Won

University of Notre Dame, Notre Dame, U.S.A.

A. Brinkerhoff, K.M. Chan, A. Drozdetskiy, M. Hildreth, C. Jessop, D.J. Karmgard, N. Kellams, K. Lannon, W. Luo, S. Lynch, N. Marinelli, T. Pearson, M. Planer, R. Ruchti,

N. Valls, M. Wayne, M. Wolf, A. Woodard

The Ohio State University, Columbus, U.S.A.

L. Antonelli, J. Brinson, B. Bylsma, L.S. Durkin, S. Flowers, C. Hill, R. Hughes, K. Kotov, T.Y. Ling, D. Puigh, M. Rodenburg, G. Smith, C. Vuosalo, B.L. Winer, H. Wolfe, H.W. Wulsin

\section{Princeton University, Princeton, U.S.A.}

E. Berry, O. Driga, P. Elmer, P. Hebda, A. Hunt, S.A. Koay, P. Lujan, D. Marlow, T. Medvedeva, M. Mooney, J. Olsen, P. Piroué, X. Quan, H. Saka, D. Stickland², C. Tully, J.S. Werner, S.C. Zenz, A. Zuranski

University of Puerto Rico, Mayaguez, U.S.A.

E. Brownson, H. Mendez, J.E. Ramirez Vargas

Purdue University, West Lafayette, U.S.A.

E. Alagoz, V.E. Barnes, D. Benedetti, G. Bolla, D. Bortoletto, M. De Mattia, A. Everett, Z. Hu, M.K. Jha, M. Jones, K. Jung, M. Kress, N. Leonardo, D. Lopes Pegna, V. Maroussov, P. Merkel, D.H. Miller, N. Neumeister, B.C. Radburn-Smith, I. Shipsey, D. Silvers, A. Svyatkovskiy, F. Wang, W. Xie, L. Xu, H.D. Yoo, J. Zablocki, Y. Zheng

Purdue University Calumet, Hammond, U.S.A.

N. Parashar, J. Stupak

Rice University, Houston, U.S.A.

A. Adair, B. Akgun, K.M. Ecklund, F.J.M. Geurts, W. Li, B. Michlin, B.P. Padley,

R. Redjimi, J. Roberts, J. Zabel

University of Rochester, Rochester, U.S.A.

B. Betchart, A. Bodek, R. Covarelli, P. de Barbaro, R. Demina, Y. Eshaq, T. Ferbel,

A. Garcia-Bellido, P. Goldenzweig, J. Han, A. Harel, A. Khukhunaishvili, D.C. Miner, G. Petrillo, D. Vishnevskiy

The Rockefeller University, New York, U.S.A.

R. Ciesielski, L. Demortier, K. Goulianos, G. Lungu, C. Mesropian 
Rutgers, The State University of New Jersey, Piscataway, U.S.A.

S. Arora, A. Barker, J.P. Chou, C. Contreras-Campana, E. Contreras-Campana, D. Duggan, D. Ferencek, Y. Gershtein, R. Gray, E. Halkiadakis, D. Hidas, A. Lath, S. Panwalkar, M. Park, R. Patel, V. Rekovic, S. Salur, S. Schnetzer, C. Seitz, S. Somalwar, R. Stone, S. Thomas, P. Thomassen, M. Walker

University of Tennessee, Knoxville, U.S.A.

K. Rose, S. Spanier, A. York

Texas A\&M University, College Station, U.S.A.

O. Bouhali ${ }^{56}$, R. Eusebi, W. Flanagan, J. Gilmore, T. Kamon ${ }^{57}$, V. Khotilovich, V. Krutelyov, R. Montalvo, I. Osipenkov, Y. Pakhotin, A. Perloff, J. Roe, A. Rose, A. Safonov, T. Sakuma, I. Suarez, A. Tatarinov

\section{Texas Tech University, Lubbock, U.S.A.}

N. Akchurin, C. Cowden, J. Damgov, C. Dragoiu, P.R. Dudero, J. Faulkner, K. Kovitanggoon, S. Kunori, S.W. Lee, T. Libeiro, I. Volobouev

Vanderbilt University, Nashville, U.S.A.

E. Appelt, A.G. Delannoy, S. Greene, A. Gurrola, W. Johns, C. Maguire, Y. Mao, A. Melo, M. Sharma, P. Sheldon, B. Snook, S. Tuo, J. Velkovska

\section{University of Virginia, Charlottesville, U.S.A.}

M.W. Arenton, S. Boutle, B. Cox, B. Francis, J. Goodell, R. Hirosky, A. Ledovskoy, H. Li, C. Lin, C. Neu, J. Wood

Wayne State University, Detroit, U.S.A.

S. Gollapinni, R. Harr, P.E. Karchin, C. Kottachchi Kankanamge Don, P. Lamichhane

\section{University of Wisconsin, Madison, U.S.A.}

D.A. Belknap, D. Carlsmith, M. Cepeda, S. Dasu, S. Duric, E. Friis, R. Hall-Wilton, M. Herndon, A. Hervé, P. Klabbers, J. Klukas, A. Lanaro, C. Lazaridis, A. Levine, R. Loveless, A. Mohapatra, I. Ojalvo, T. Perry, G.A. Pierro, G. Polese, I. Ross, T. Sarangi, A. Savin, W.H. Smith, N. Woods

$\dagger$ : Deceased

1: Also at Vienna University of Technology, Vienna, Austria

2: Also at CERN, European Organization for Nuclear Research, Geneva, Switzerland

3: Also at Institut Pluridisciplinaire Hubert Curien, Université de Strasbourg, Université de Haute Alsace Mulhouse, CNRS/IN2P3, Strasbourg, France

4: Also at National Institute of Chemical Physics and Biophysics, Tallinn, Estonia

5: Also at Skobeltsyn Institute of Nuclear Physics, Lomonosov Moscow State University, Moscow, Russia

6: Also at Universidade Estadual de Campinas, Campinas, Brazil

7: Also at California Institute of Technology, Pasadena, U.S.A.

8: Also at Laboratoire Leprince-Ringuet, Ecole Polytechnique, IN2P3-CNRS, Palaiseau, France 
9: Also at Suez University, Suez, Egypt

10: Also at British University in Egypt, Cairo, Egypt

11: Also at Fayoum University, El-Fayoum, Egypt

12: Now at Ain Shams University, Cairo, Egypt

13: Also at Université de Haute Alsace, Mulhouse, France

14: Also at Joint Institute for Nuclear Research, Dubna, Russia

15: Also at Brandenburg University of Technology, Cottbus, Germany

16: Also at The University of Kansas, Lawrence, U.S.A.

17: Also at Institute of Nuclear Research ATOMKI, Debrecen, Hungary

18: Also at Eötvös Loránd University, Budapest, Hungary

19: Also at University of Debrecen, Debrecen, Hungary

20: Also at Tata Institute of Fundamental Research - HECR, Mumbai, India

21: Now at King Abdulaziz University, Jeddah, Saudi Arabia

22: Also at University of Visva-Bharati, Santiniketan, India

23: Also at University of Ruhuna, Matara, Sri Lanka

24: Also at Isfahan University of Technology, Isfahan, Iran

25: Also at Sharif University of Technology, Tehran, Iran

26: Also at Plasma Physics Research Center, Science and Research Branch, Islamic Azad University, Tehran, Iran

27: Also at Laboratori Nazionali di Legnaro dell'INFN, Legnaro, Italy

28: Also at Università degli Studi di Siena, Siena, Italy

29: Also at Centre National de la Recherche Scientifique (CNRS) - IN2P3, Paris, France

30: Also at Purdue University, West Lafayette, U.S.A.

31: Also at Universidad Michoacana de San Nicolas de Hidalgo, Morelia, Mexico

32: Also at National Centre for Nuclear Research, Swierk, Poland

33: Also at Institute for Nuclear Research, Moscow, Russia

34: Also at St. Petersburg State Polytechnical University, St. Petersburg, Russia

35: Also at Faculty of Physics, University of Belgrade, Belgrade, Serbia

36: Also at Facoltà Ingegneria, Università di Roma, Roma, Italy

37: Also at Scuola Normale e Sezione dell'INFN, Pisa, Italy

38: Also at University of Athens, Athens, Greece

39: Also at Paul Scherrer Institut, Villigen, Switzerland

40: Also at Institute for Theoretical and Experimental Physics, Moscow, Russia

41: Also at Albert Einstein Center for Fundamental Physics, Bern, Switzerland

42: Also at Gaziosmanpasa University, Tokat, Turkey

43: Also at Adiyaman University, Adiyaman, Turkey

44: Also at Cag University, Mersin, Turkey

45: Also at Mersin University, Mersin, Turkey

46: Also at Izmir Institute of Technology, Izmir, Turkey

47: Also at Ozyegin University, Istanbul, Turkey

48: Also at Kafkas University, Kars, Turkey

49: Also at Mimar Sinan University, Istanbul, Istanbul, Turkey

50: Also at Rutherford Appleton Laboratory, Didcot, United Kingdom

51: Also at School of Physics and Astronomy, University of Southampton, Southampton, United Kingdom

52: Also at University of Belgrade, Faculty of Physics and Vinca Institute of Nuclear Sciences, Belgrade, Serbia

53: Also at Argonne National Laboratory, Argonne, U.S.A. 
54: Also at Erzincan University, Erzincan, Turkey

55: Also at Yildiz Technical University, Istanbul, Turkey

56: Also at Texas A\&M University at Qatar, Doha, Qatar

57: Also at Kyungpook National University, Daegu, Korea 